Supporting information

\title{
Diaminomethylenemalononitriles and diaminomethyleneindanediones as dual hydrogen bond donors for anion recognition
}

Jakob D. E. Lane ${ }^{a}$, Stuart N. Berry ${ }^{a}$, William Lewis ${ }^{a, b}$, Junming Hoc and Katrina A. Jolliffe ${ }^{a,{ }^{*}}$

aschool of Chemistry, The University of Sydney, NSW, 2006, Australia

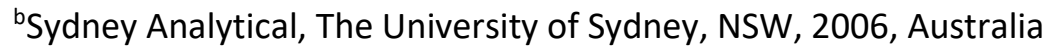

'School of Chemistry, University of New South Wales, NSW, 2052, Australia 


\section{Contents}

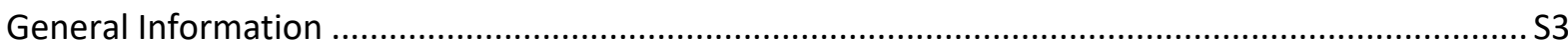

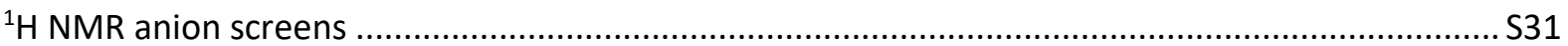

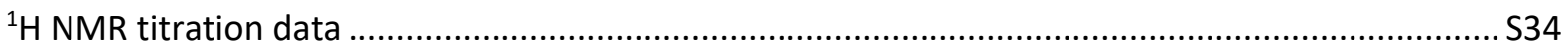

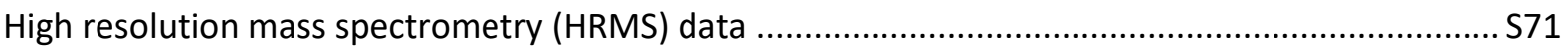

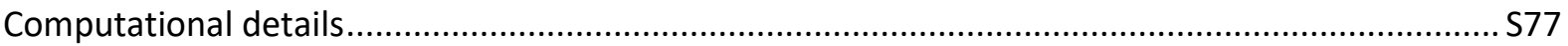

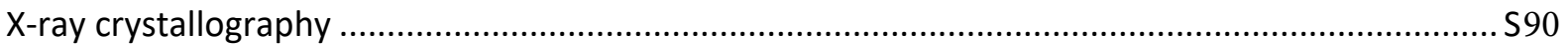

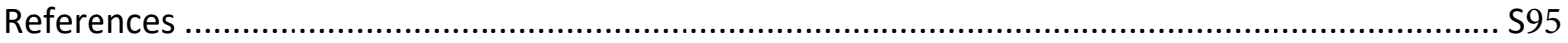




\section{General Information}

Nuclear magnetic resonance (NMR) spectra were recorded at $300 \mathrm{~K}$ using either a Bruker Avance DPX 400 or a Bruker Avance 300 spectrometer. ${ }^{1} \mathrm{H}$ NMR and ${ }^{13} \mathrm{C}\left\{{ }^{1} \mathrm{H}\right\}$ NMR spectra were recorded at the indicated frequencies. Chemical shifts are expressed as parts per million (ppm) and are referenced to solvent residual signals. The data are reported as chemical shift, multiplicity (br = broad, $\mathrm{s}=$ singlet, $\mathrm{d}=$ doublet, $\mathrm{t}=$ triplet, $\mathrm{m}=$ multiplet), coupling constant $\mathrm{J}$ in $\mathrm{Hz}$ and relative integral. Low resolution mass spectra were recorded on a Bruker amaZon SL mass spectrometer using electrospray ionisation (ESI, positive or negative mode). High resolution mass spectra were recorded on a Bruker Apex II Fourier Transform Ion Cyclotron Resonance (FTICR) mass spectrometer with a 7.0 T magnet, fitted with an off-axis Analytic electrospray source and are reported as $\mathrm{m} / \mathrm{z}$ (relative intensity). Infrared (IR) absorption spectra were recorded on a Bruker Alpha-E FT-IR spectrometer using attenuated total reflection (ATR) of either a solid or a thin film. Notable vibrational wavenumbers are recorded in $\mathrm{cm}^{-1}$.

All reagents were used as received from commercial sources unless otherwise noted. Tetrahydrofuran, acetonitrile, methanol, dichloromethane, and dimethylformamide were purified using an Innovative Technology, Inc., Pure-solve solvent purification system.

NMR titrations were performed by additions of aliquots of the putative anionic guest as the tetrabutylammonium (TBA) salt (0.1-0.2 M) made up in a solution of the receptor (2.5-4.0 mM) in either MeCN- $d_{3}$ or DMSO- $d_{6}$ (0.5\% water). Both the anion salt and receptor were dried under high vacuum over $\mathrm{P}_{4} \mathrm{O}_{10}$ to remove residual solvent and water, respectively. ${ }^{1} \mathrm{H}$ NMR spectra were recorded on a Bruker Avance DPX 500 spectrometer or a Bruker Avance DPX 400 spectrometer and calibrated to the residual proton solvent peak in $\mathrm{MeCN}-\mathrm{d}_{3}(\delta=1.94 \mathrm{ppm})$ or DMSO- $\mathrm{d}_{6}(\delta=2.50 \mathrm{ppm})$ at $300 \mathrm{~K}$. Stack plots were made using MestReNova Version 6.0. Where possible, non-linear leastsquare curve fitting of the titration data to a $1: 1$ binding model using Bindfit ${ }^{1,2}$ v0.5 enabled the calculation of association constants $\left(K_{\mathrm{a}}\right)$. The obtained $K_{\mathrm{a}}$ values represent the average of two independent titrations. The fitting error from Bindfit is shown for each $\mathrm{K}_{\mathrm{a}}$ values, and experimental error is estimated at less than $15 \%$ for each $\mathrm{K}_{\mathrm{a}}$ value obtained. 


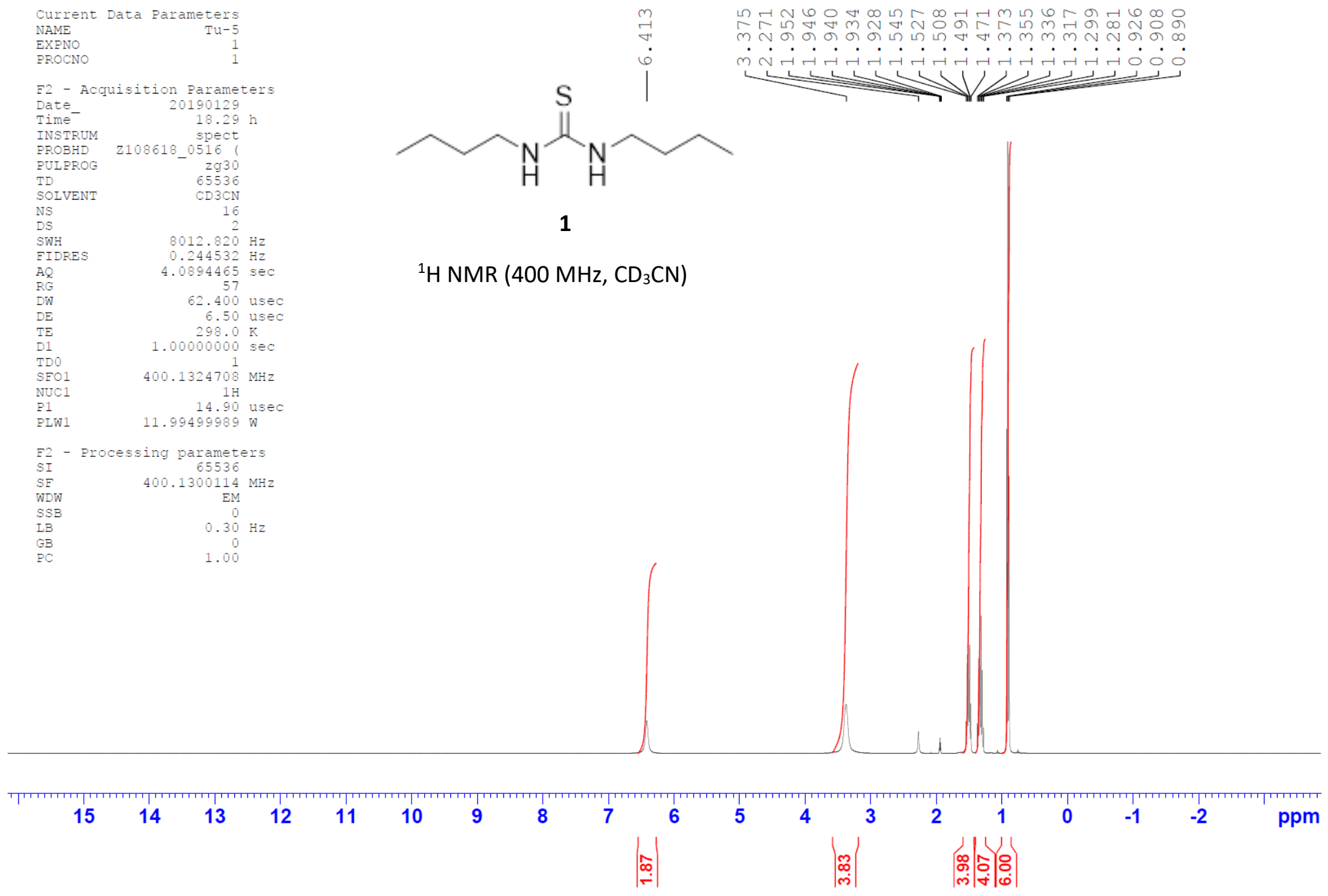




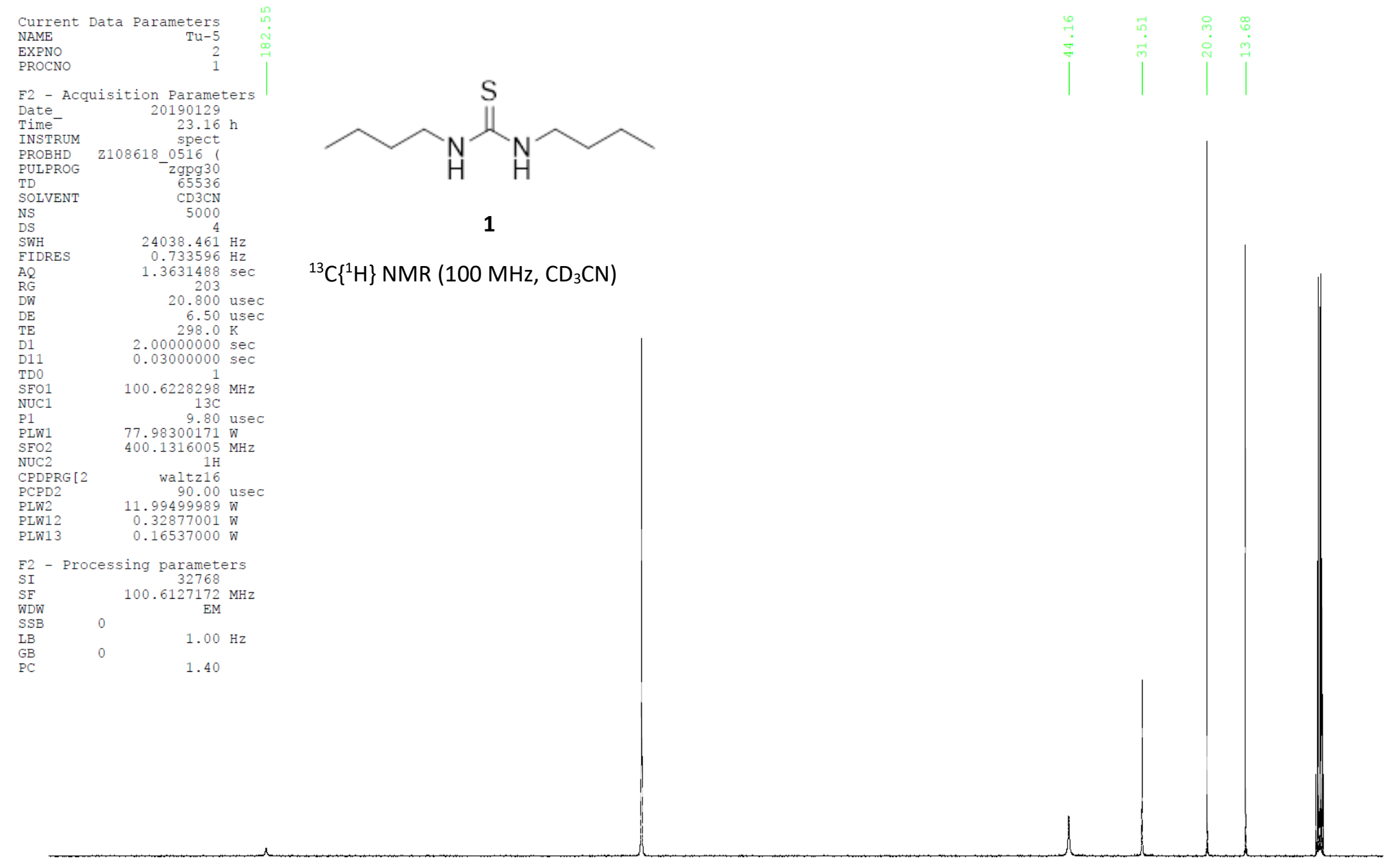

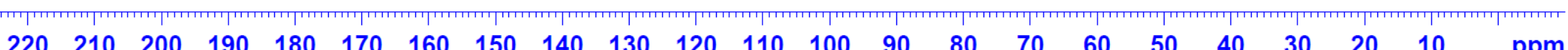




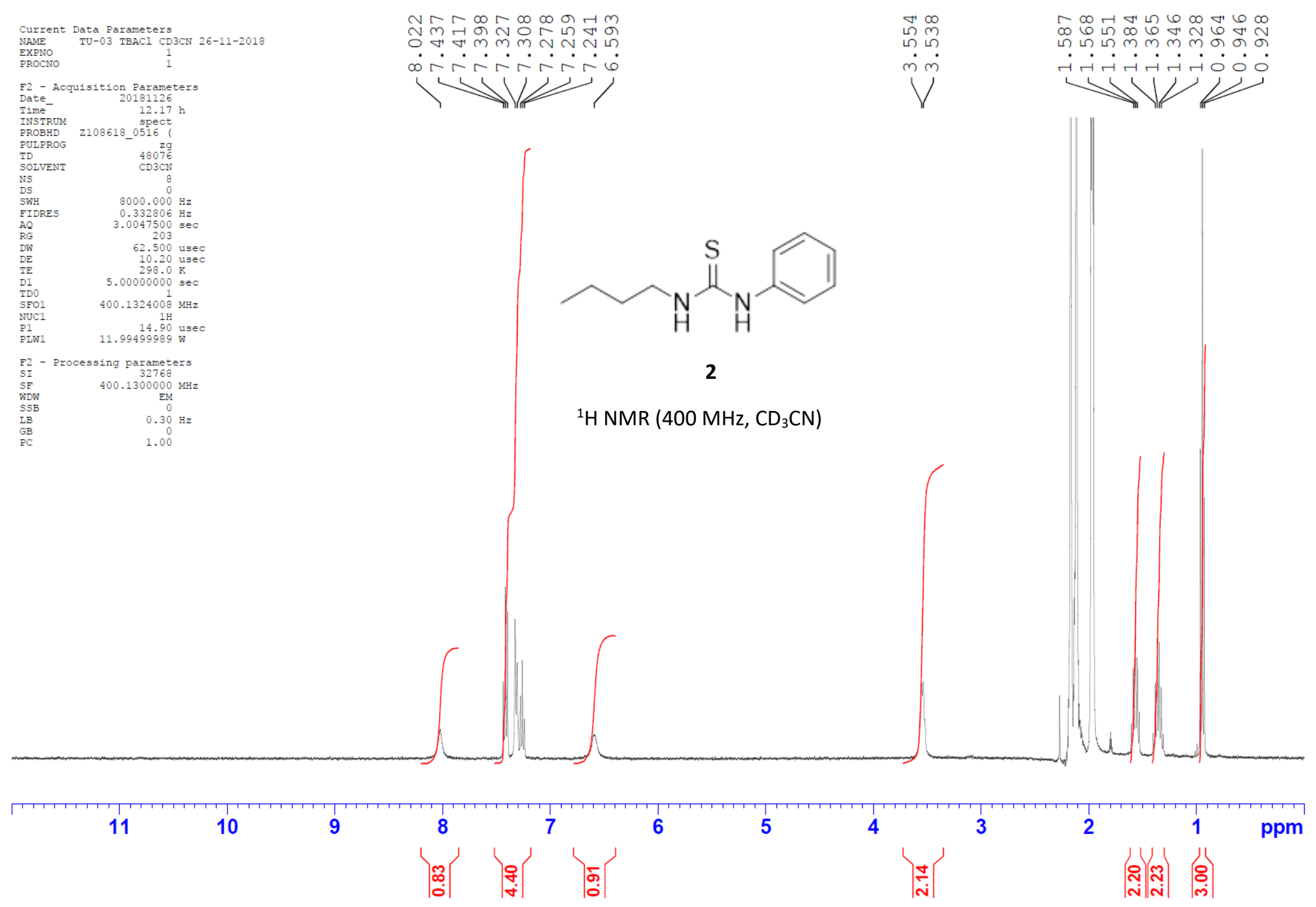


Current Data Parameters
NAME TU-03 carbon

NAME
EXPNO
PROCNO

F2 - Acquisition Parameters

$\begin{array}{ll}\text { Date_rime } & 20190118 \\ \text { Time } & 1023\end{array}$

INSTRUM spect

PROBHD
PULPROG

TD

NS

$\begin{array}{lr}\text { DS } & 4 \\ \text { SWH } & 25252.525 \mathrm{~Hz} \\ \text { FIDRES } & 0.770646 \mathrm{~Hz}\end{array}$

AQ $\quad 1.2976128 \mathrm{~Hz}$

$\begin{array}{lr}\text { RG } & 203 \\ \text { DW } & 19.800 \text { usec } \\ \text { DE } & 6.50 \text { usec } \\ \text { TE } & 298.1 \mathrm{~K}\end{array}$

$\begin{array}{ll}\text { TE } & 2.000008 .1 \mathrm{~K} \\ \text { D1 } & 0.000000000 \mathrm{sec} \\ \text { D11 } & 0.03000000 \mathrm{sec}\end{array}$

$\begin{array}{lr}\text { TD0 } & 25 \\ \text { SFO1 } & 100.6228303 \mathrm{MHz}\end{array}$

NUC1
P1

$\begin{array}{ll}\text { PLW1 } & 77.98300171 \mathrm{~W} \\ \text { SFO2 } & 400.1316005 \mathrm{MHz}\end{array}$

$\begin{array}{lr}\text { NUC2 } & 1 \mathrm{H} \\ \text { CPDPRG [2 waltz16 } & \end{array}$

$\begin{array}{ll}\text { PCPD2 } & 90.00 \text { usec } \\ \text { PIW2 } & 90.99409\end{array}$

$\begin{array}{lr}\text { PLW2 } & 11.99499989 \mathrm{~W} \\ \text { PLW12 } & 0.32877001 \mathrm{~W}\end{array}$

F2 - Processing parameters

$\underset{\mathrm{SI}}{\mathrm{F} 2}$ - Processing parameters

WDW $\quad 100.612790 \mathrm{MHz}$

$\begin{array}{lll}\text { SSB } & 0 & 1.00 \mathrm{~Hz} \\ \mathrm{IB} & 0 & 1.40 \\ \text { GB } & 0\end{array}$

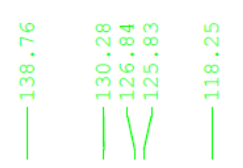

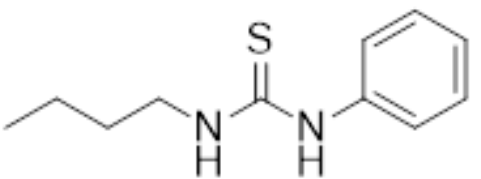

2

${ }^{13} \mathrm{C}\left\{{ }^{1} \mathrm{H}\right\}$ NMR $\left(100 \mathrm{MHz}, \mathrm{CD}_{3} \mathrm{CN}\right)$

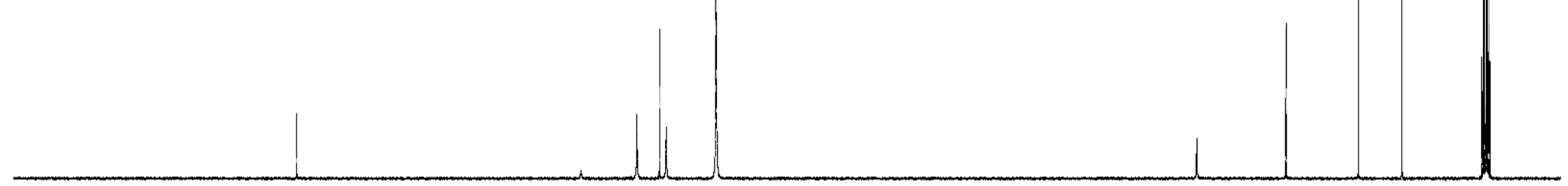




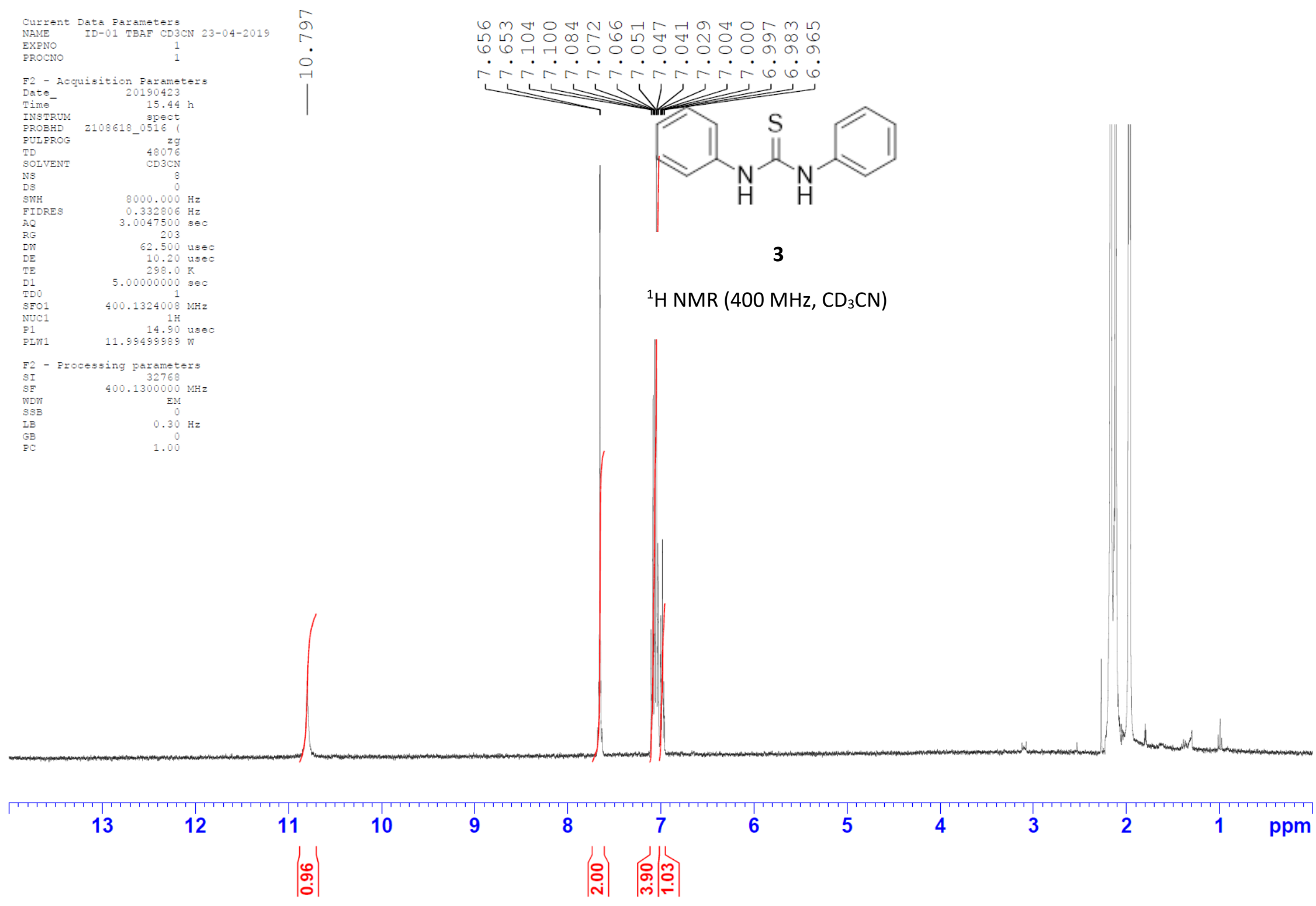



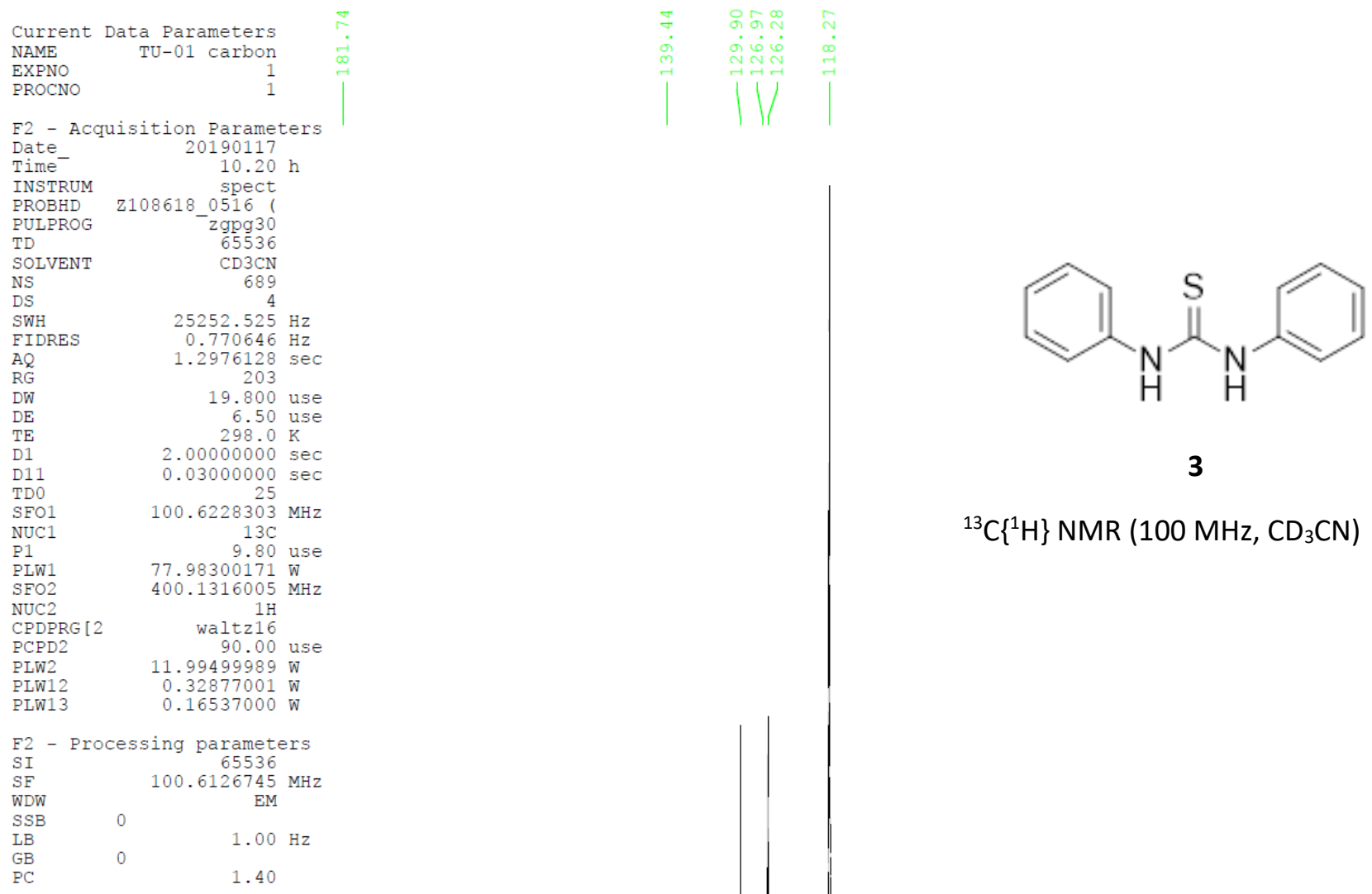

3

${ }^{13} \mathrm{C}\left\{{ }^{1} \mathrm{H}\right\}$ NMR $\left(100 \mathrm{MHz}, \mathrm{CD}_{3} \mathrm{CN}\right)$

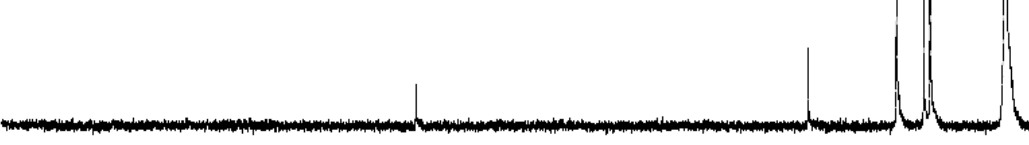

$\begin{array}{lllllllllll}220 & 210 & 200 & 190 & 180 & 170 & 160 & 150 & 140 & 130 & 120\end{array}$

$\begin{array}{lll}120 & 110 & 100\end{array}$

$80 \quad 70$

60

50

$40 \quad 30$

$20 \quad 10$

ppm 


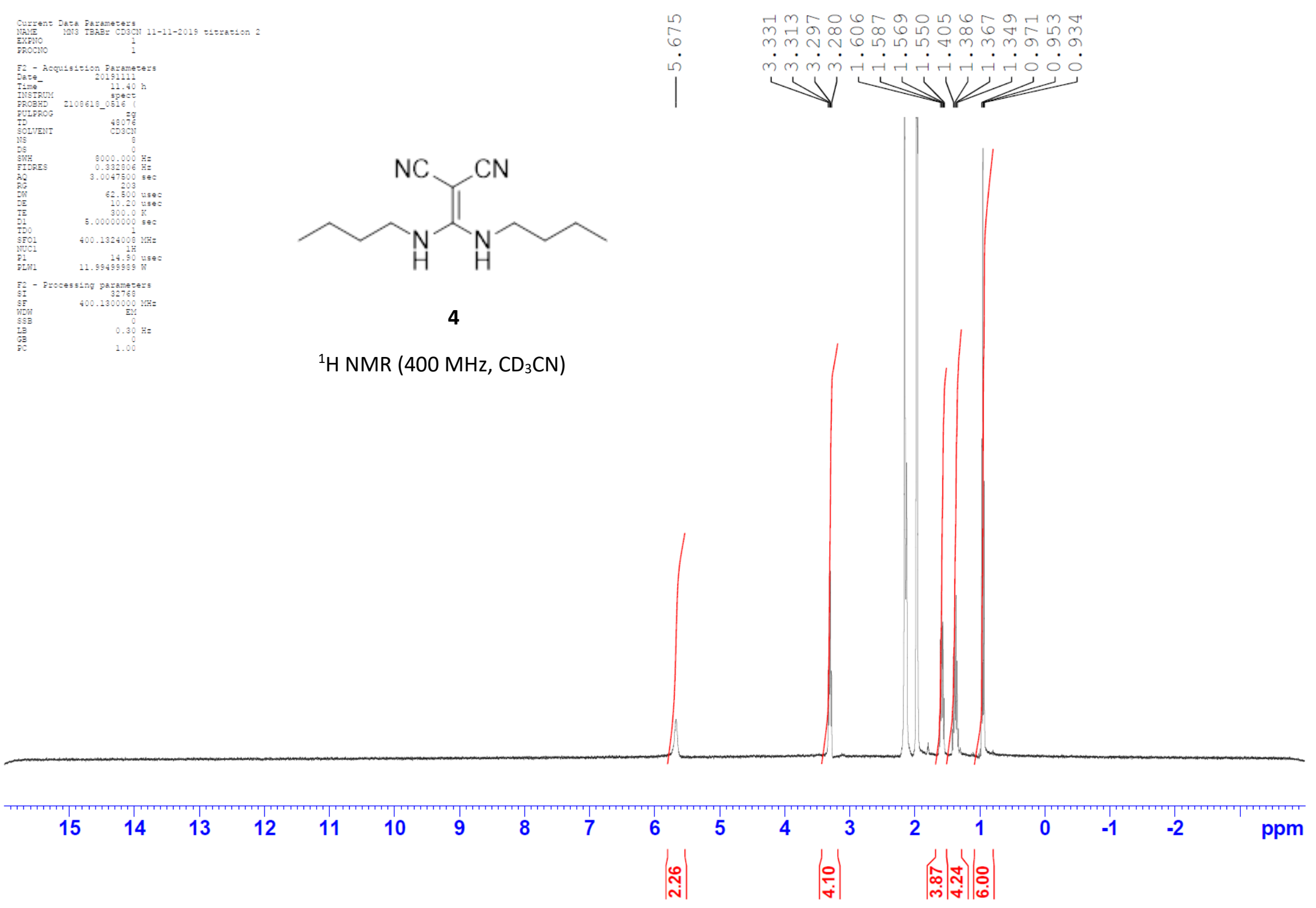




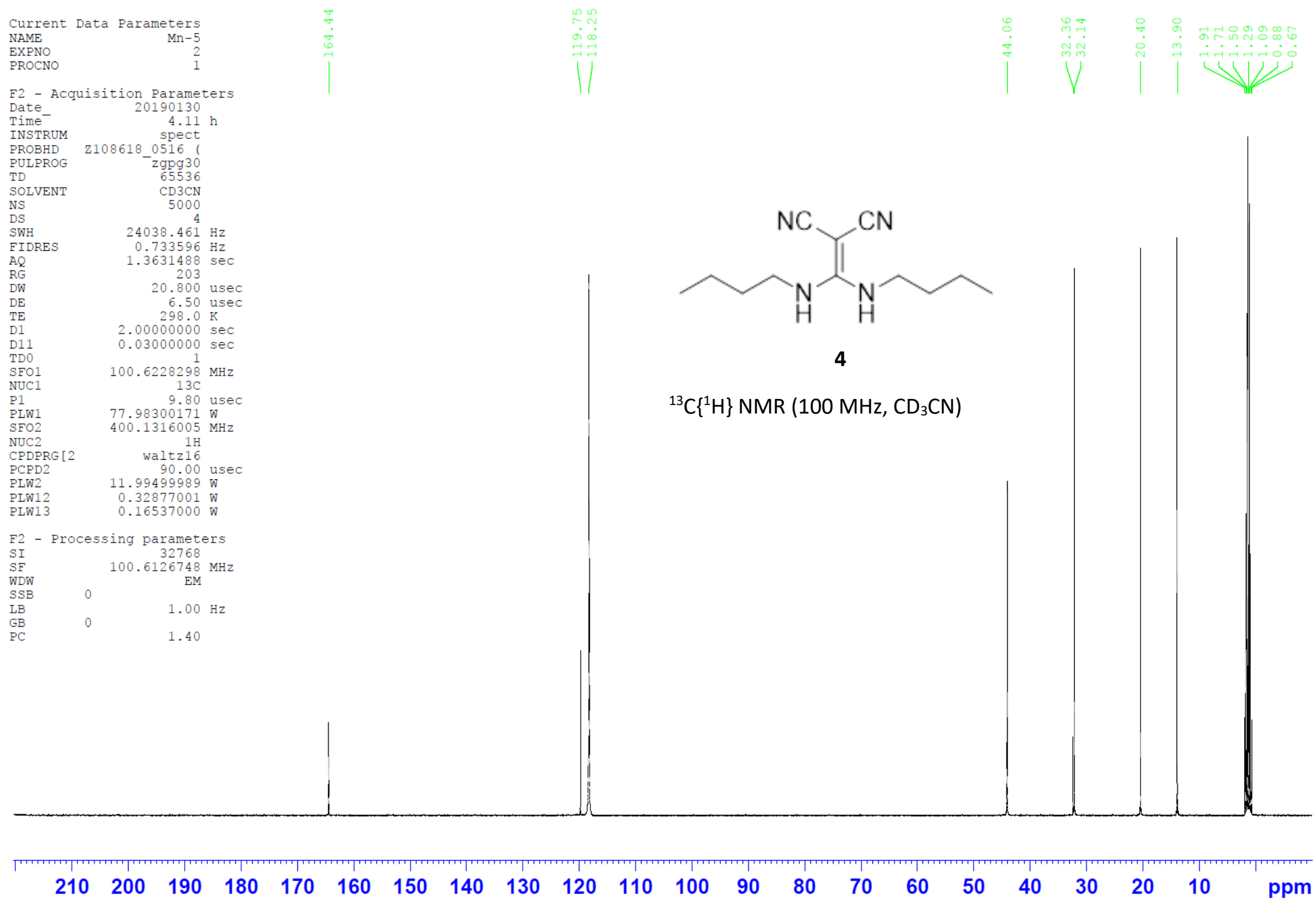




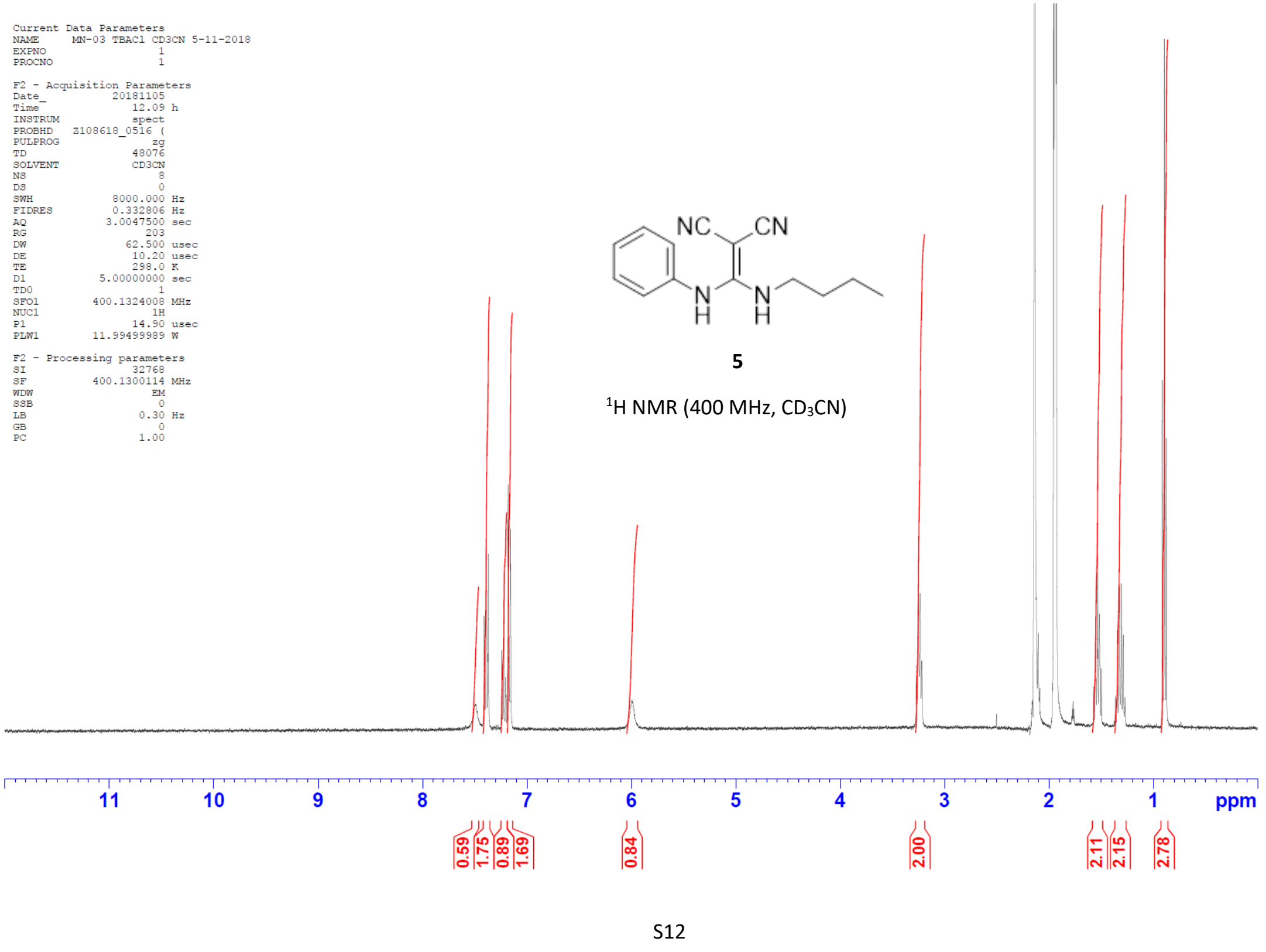




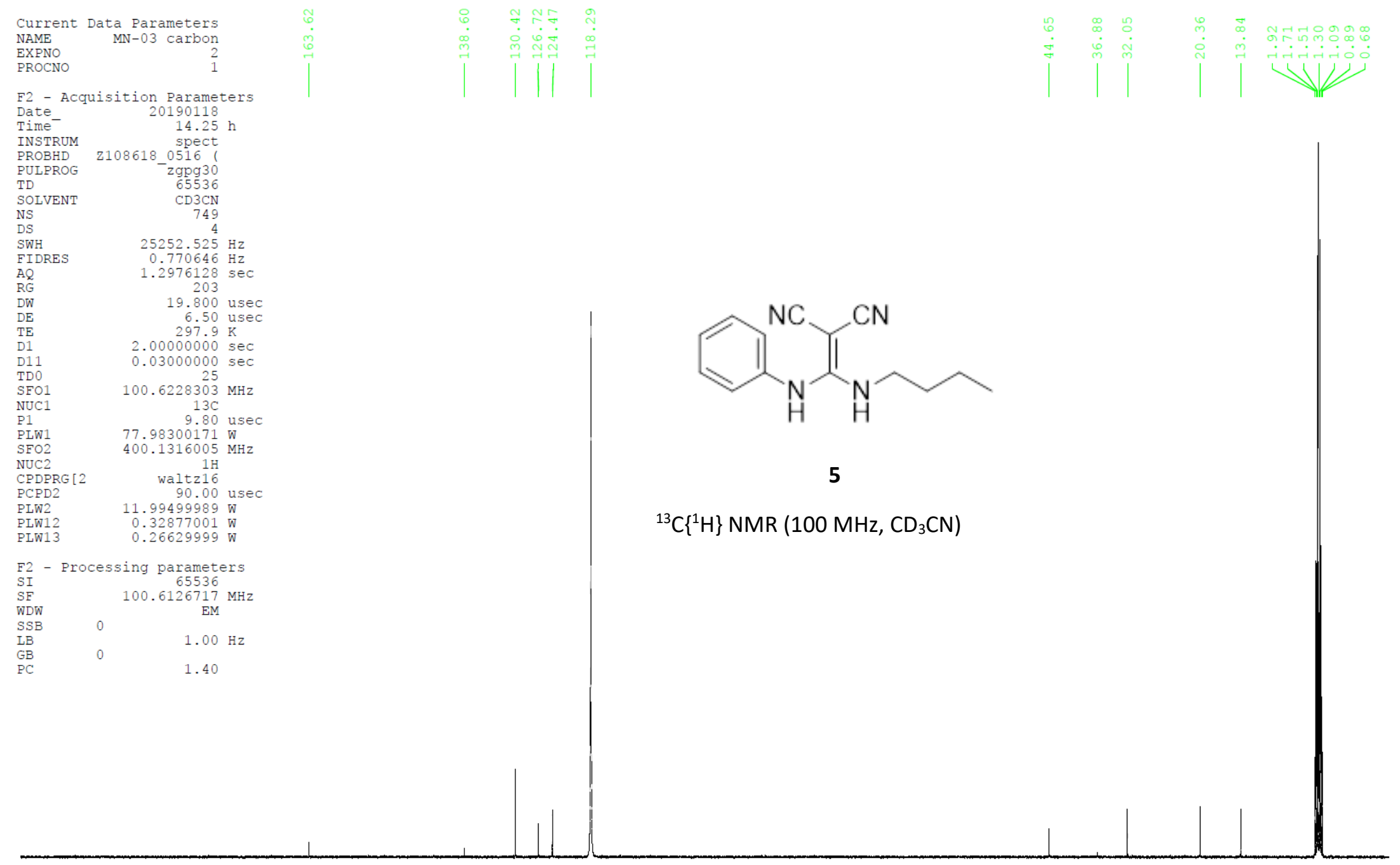

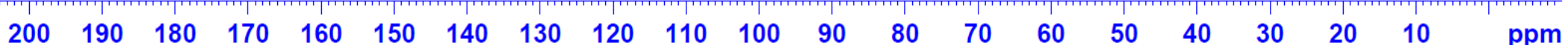




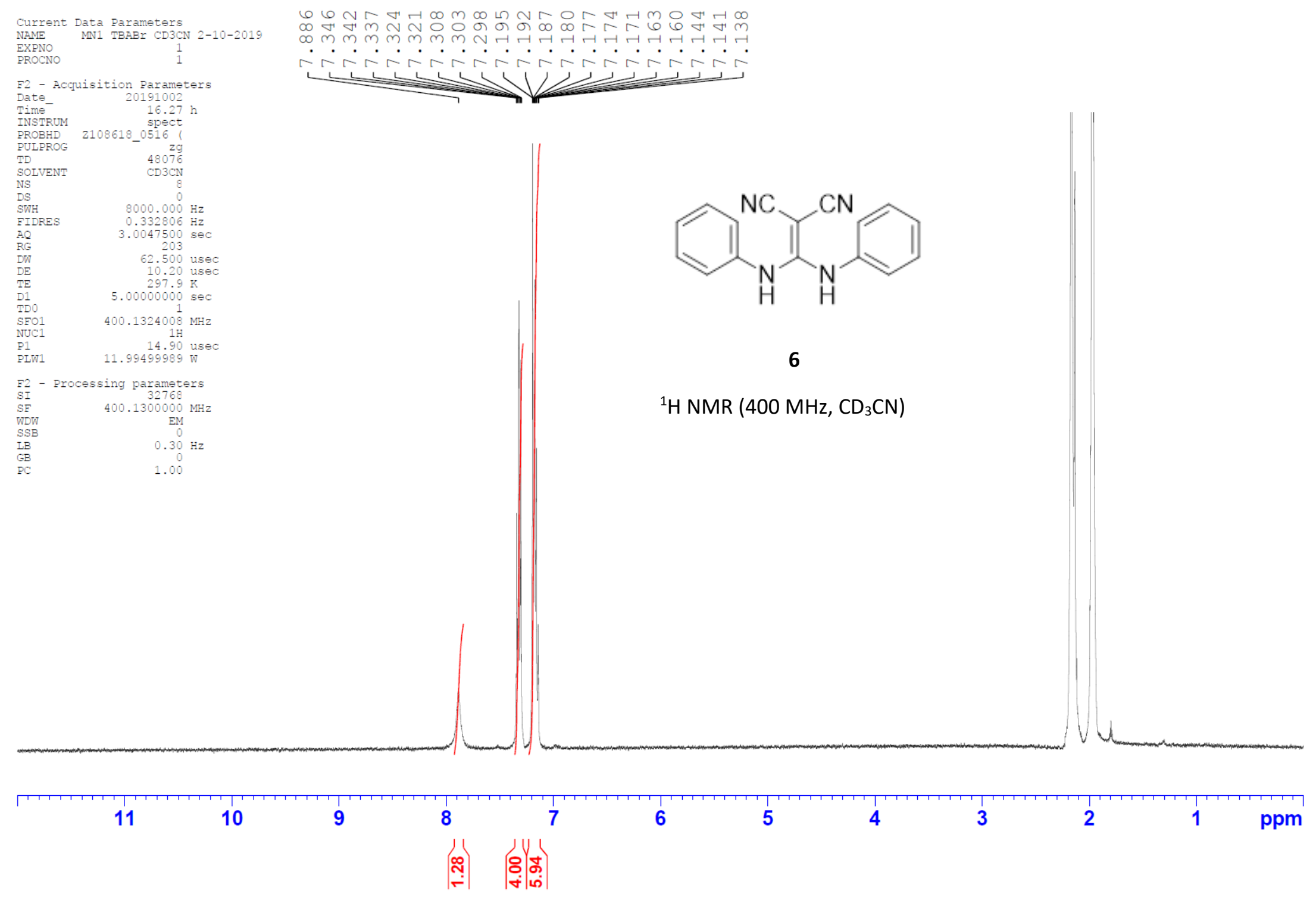


Current Data Parameters

NAME

EXPNO
PROCNO

F2 - Acquisition Parameter

Date- 20190118 . 203

INSTRUM

PROBHD 2108618 0516 spect

PULPROG

TD
SOLVENT

NS

\begin{tabular}{lr} 
DS & \multicolumn{1}{c}{4} \\
SWH & $25252.525 \mathrm{~Hz}$ \\
FIDRES & $0.770646 \mathrm{~Hz}$
\end{tabular}

$\begin{array}{ll}\mathrm{AQ} & 1.2976128 \mathrm{sec} \\ \mathrm{RG} & \end{array}$

DW $\quad 19.800$ usec

$\begin{array}{lr}\text { DE } & 6.50 \mathrm{usec} \\ \text { TE } & 298.0 \mathrm{~K}\end{array}$

$\begin{array}{ll}\text { D1 } & 2.00000000 \mathrm{sec} \\ \text { D11 } & 0.03000000 \mathrm{sec}\end{array}$

25

$\begin{array}{lr}\text { SFO1 } & 100.6228303 \mathrm{MHz} \\ \text { NUC1 } & 13 \mathrm{C}\end{array}$

$\begin{array}{ll}\text { P1 } & 9.80 \text { usec } \\ \text { PLW1 } & 77.98300171 \mathrm{~W}\end{array}$

400.1316005 MH

CPDPRG [2

PCPD2

PLW2 2
PLW12$$
\text { waltz16 }
$$

90.00 usec

$11.99499989 \mathrm{~W}$
$0.32877001 \mathrm{~W}$

F2 - Processing parameters

$\begin{array}{ll}\text { SI } & 65536 \\ \text { SF } & 100.6126708 \mathrm{MHz}\end{array}$

WSB 0

$\begin{array}{lll}\mathrm{LB} & & 1.00 \mathrm{HZ}\end{array}$

PC $\quad 1.40$

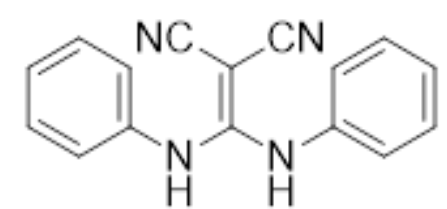

${ }^{13} \mathrm{C}\left\{{ }^{1} \mathrm{H}\right\}$ NMR $\left(100 \mathrm{MHz}, \mathrm{CD}_{3} \mathrm{CN}\right)$
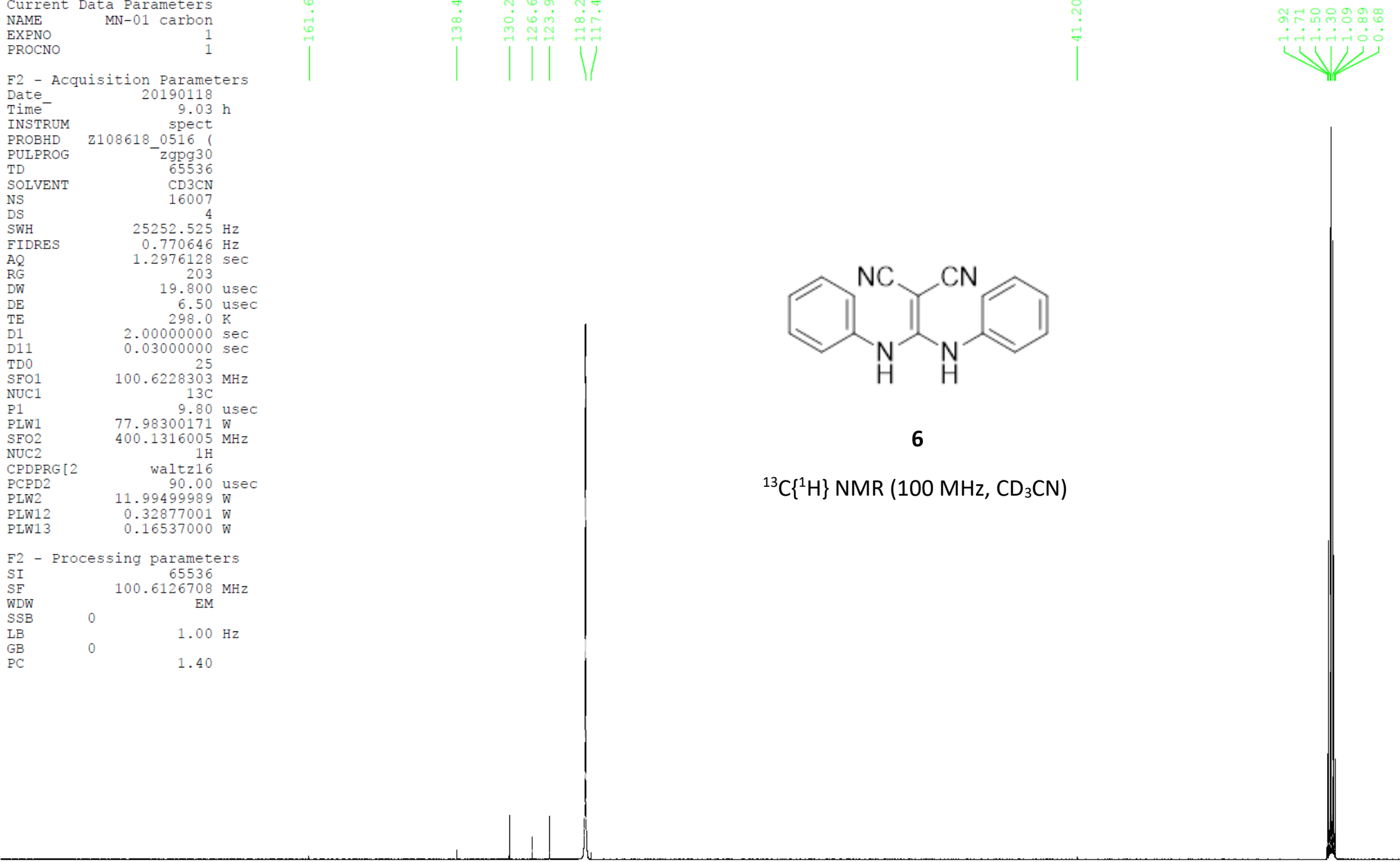


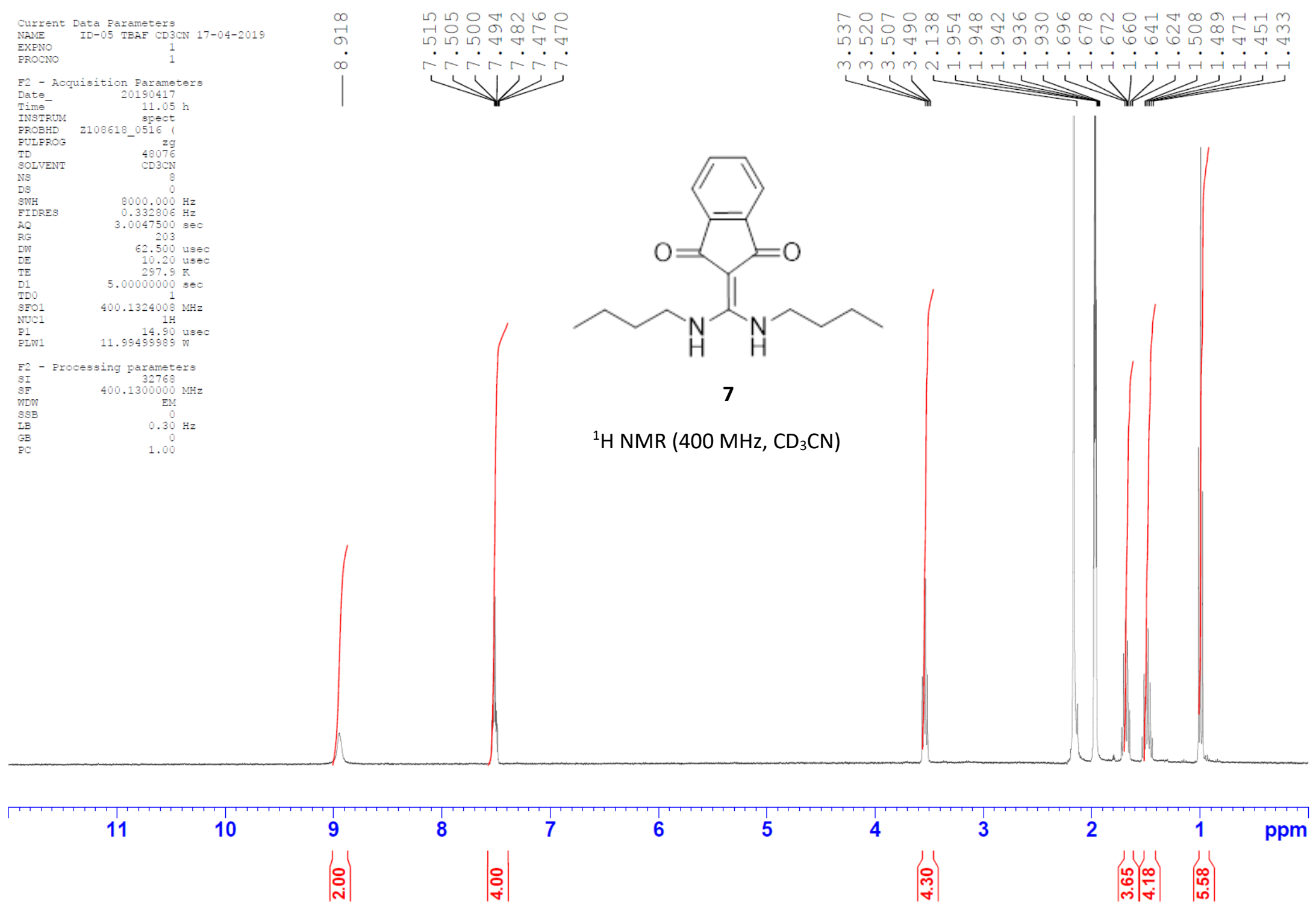


Current Data Parameters

NAME
EXPNO
PROCNO

F2 - Acquisition Parameter

$\begin{array}{lr}\text { Date } & 20190124 \\ \text { Time } & 1056 \mathrm{~h}\end{array}$

spect
INSTRUM

PROBHD 2108618

Tgpg30
TD
SOLVENT
CD $356 \mathrm{CN}$

$\begin{array}{lr}\text { DS } & 4 \\ \text { SWH } & 25252.525 \mathrm{~Hz} \\ \text { FIDRES } & 0.770646 \mathrm{~Hz}\end{array}$

$\begin{array}{lll}A Q & 1.2976128 \mathrm{~Hz} \\ \mathrm{RG} & \mathrm{sec}\end{array}$

$\begin{array}{lr}\text { RG } & 19.800 \text { usec } \\ \text { DW } & 6.50 \text { usec } \\ \text { DE } & 6.000 \text { sec }\end{array}$

$\begin{array}{ll}\text { TE } & 298.0 \mathrm{~K} \\ \mathrm{D} 1 & 2.0000000 \mathrm{sec}\end{array}$

$\begin{array}{ll}\text { D1 } & 2.00000000 \mathrm{sec} \\ \text { D11 } & 0.03000000 \mathrm{sec}\end{array}$

TFO $\quad 100.6228303 \mathrm{MHz}$

NuCl $13 \mathrm{C}$

$\begin{array}{ll}\text { P1 } & 77.98300 .80 \text { usec } \\ \text { PLW1 } & 400.1316005 \mathrm{WHz}\end{array}$

NUC2 2000

PCPD2 waltz16

$\begin{array}{lr}\text { PLW2 } & 11.99499989 \mathrm{~W} \\ \text { PLW12 } & 0.32877001 \mathrm{~W}\end{array}$

$0.326537000 \mathrm{~W}$

F2 - Processing parameters

SI $\quad 100.6126732 \mathrm{MHz}$

$\begin{array}{lc}\text { SF } & 100.6126732 \\ \text { WDW } & \text { MM }\end{array}$

$\begin{array}{lll}\mathrm{SSB} & 0 & 1.00 \mathrm{~Hz}\end{array}$

$\begin{array}{lll}\mathrm{GB} & 0 & 1.40\end{array}$

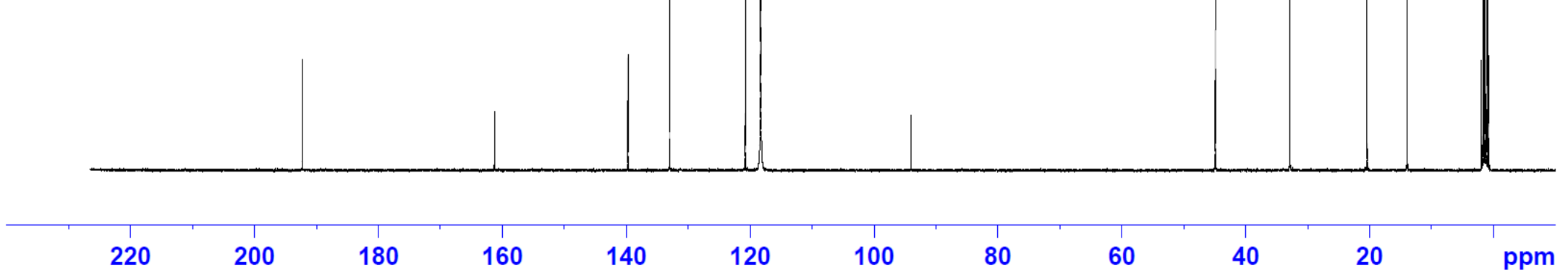

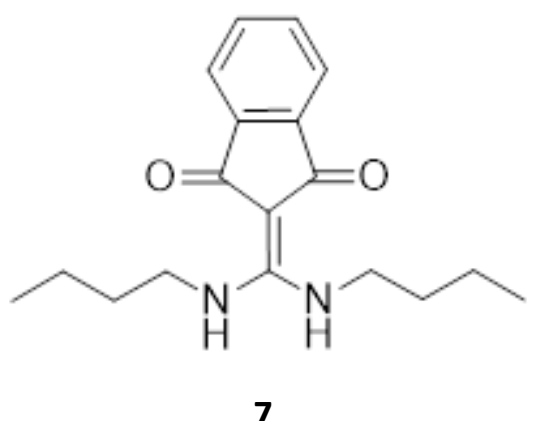

${ }^{13} \mathrm{C}\left\{{ }^{1} \mathrm{H}\right\}$ NMR $\left(100 \mathrm{MHz}, \mathrm{CD}_{3} \mathrm{CN}\right)$
100

80

60

40

20

ppm 


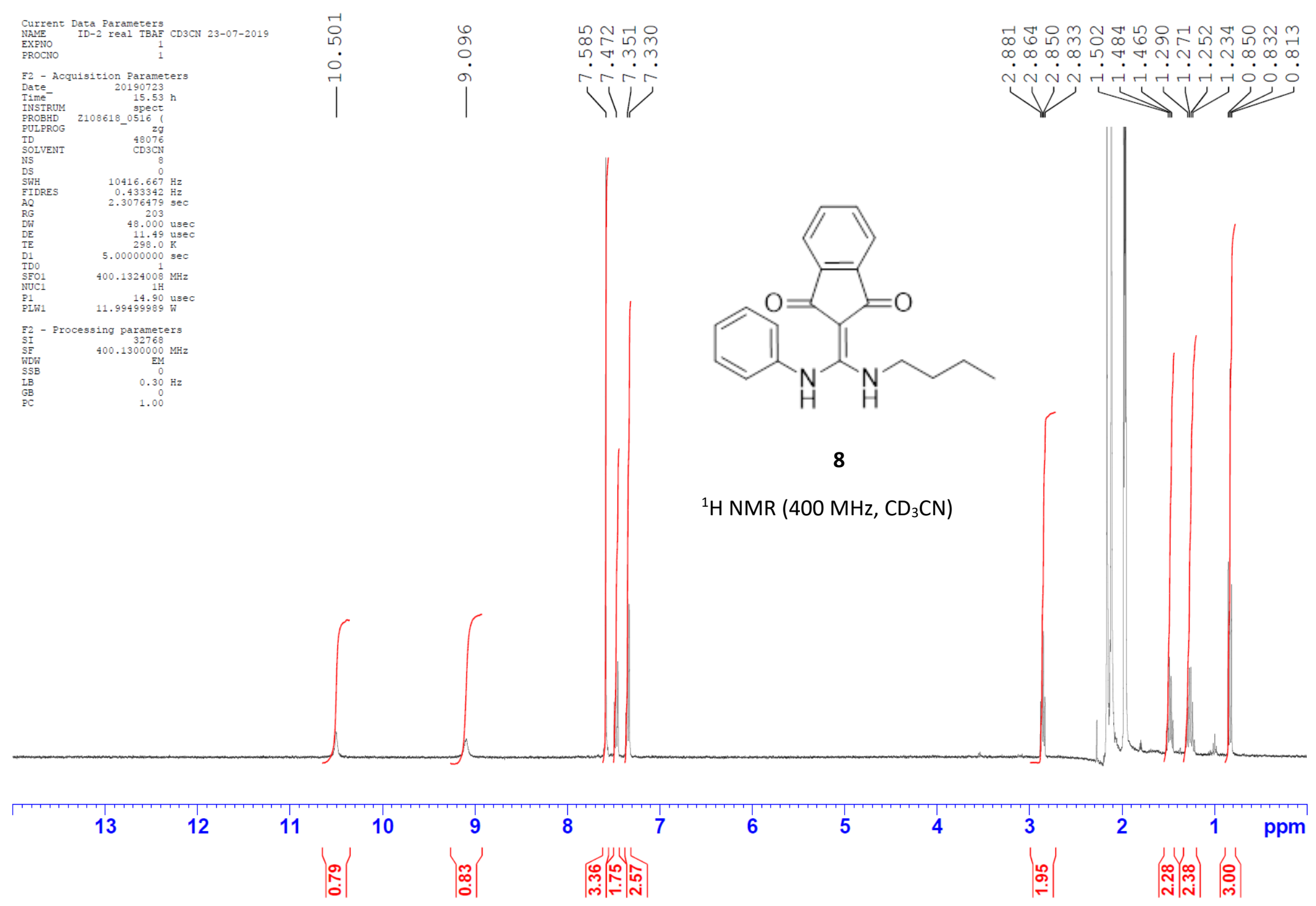


Current Data Parameters
NAME ID-02 real after column EXPNO
PROCNO
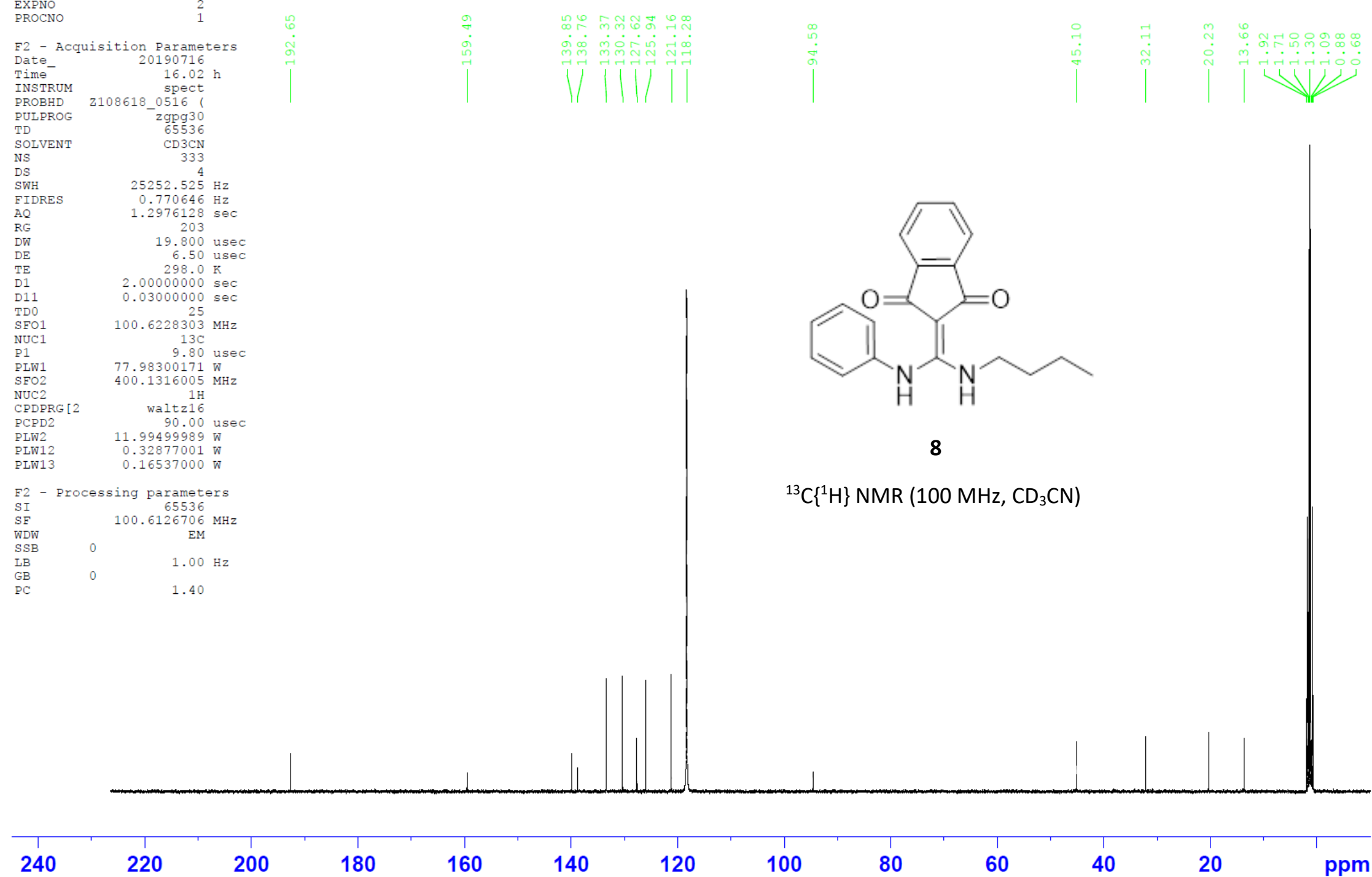


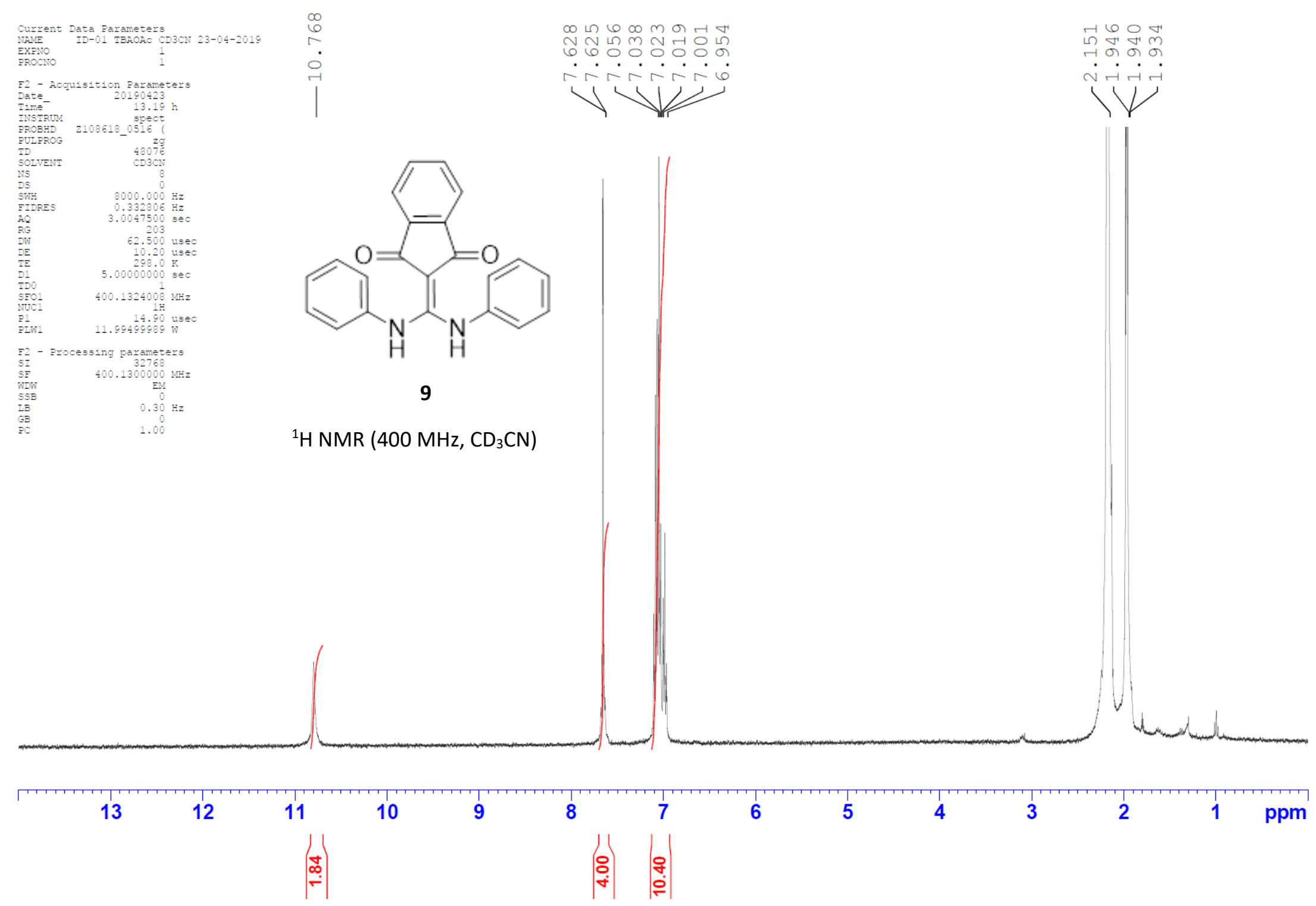




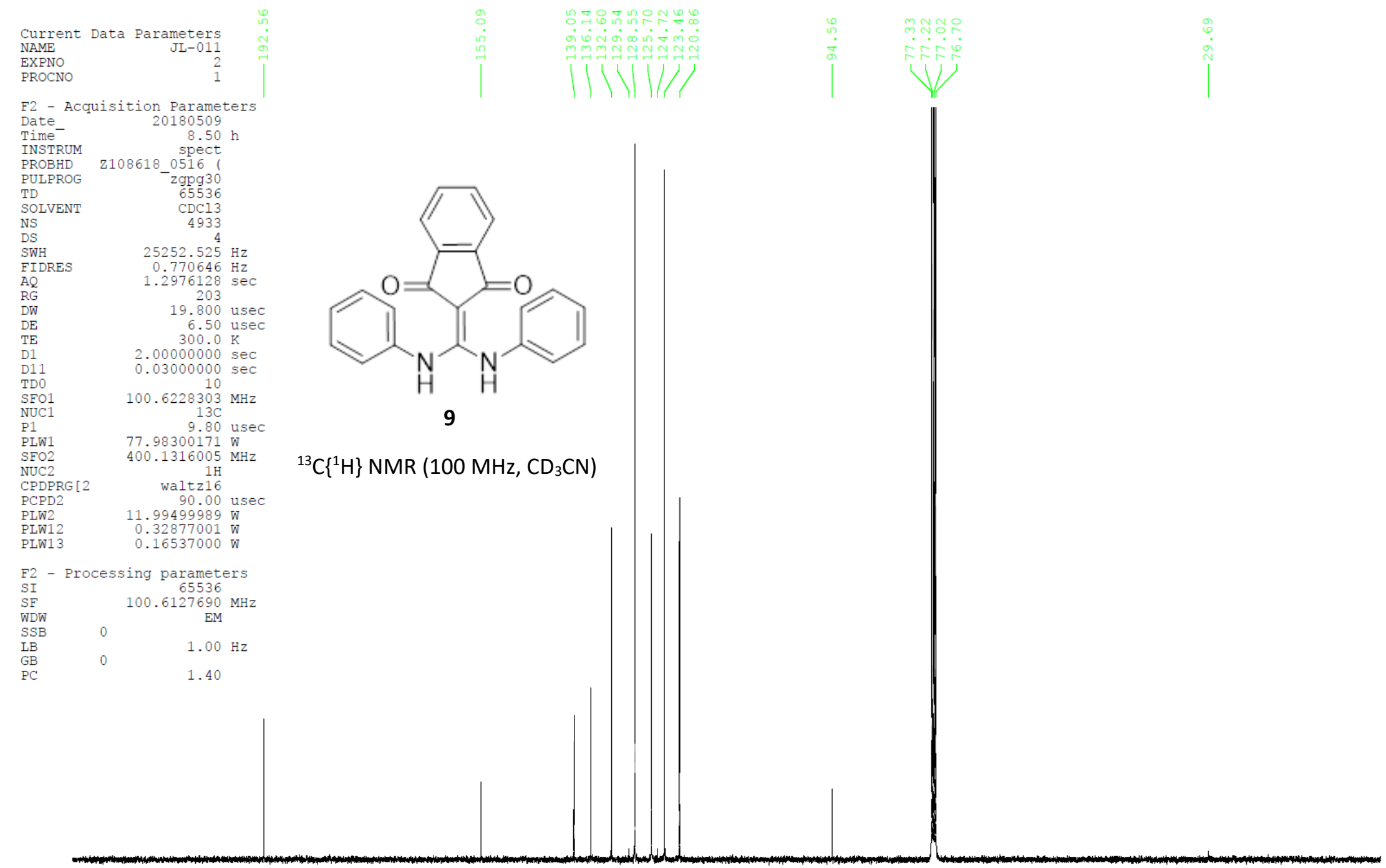




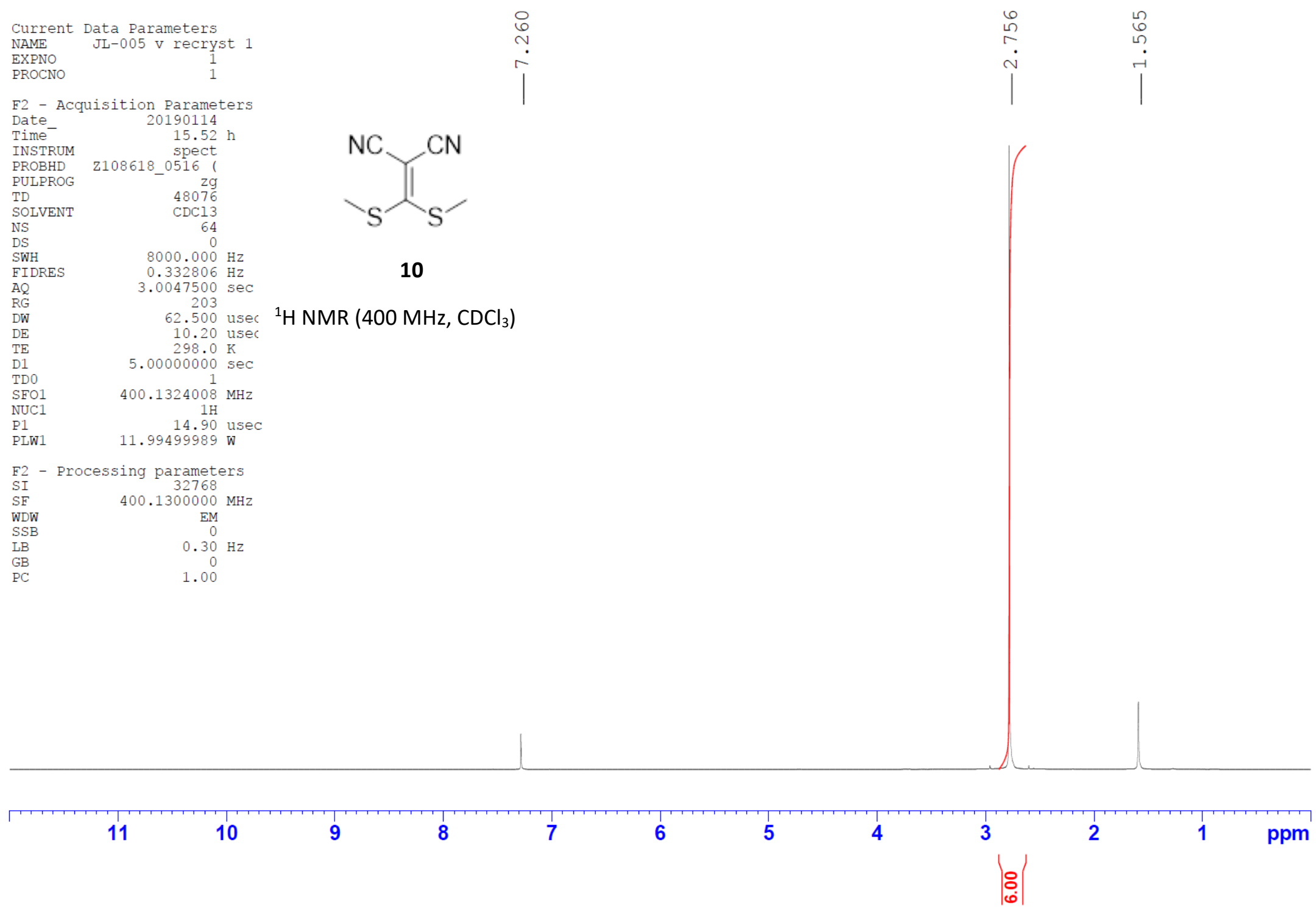




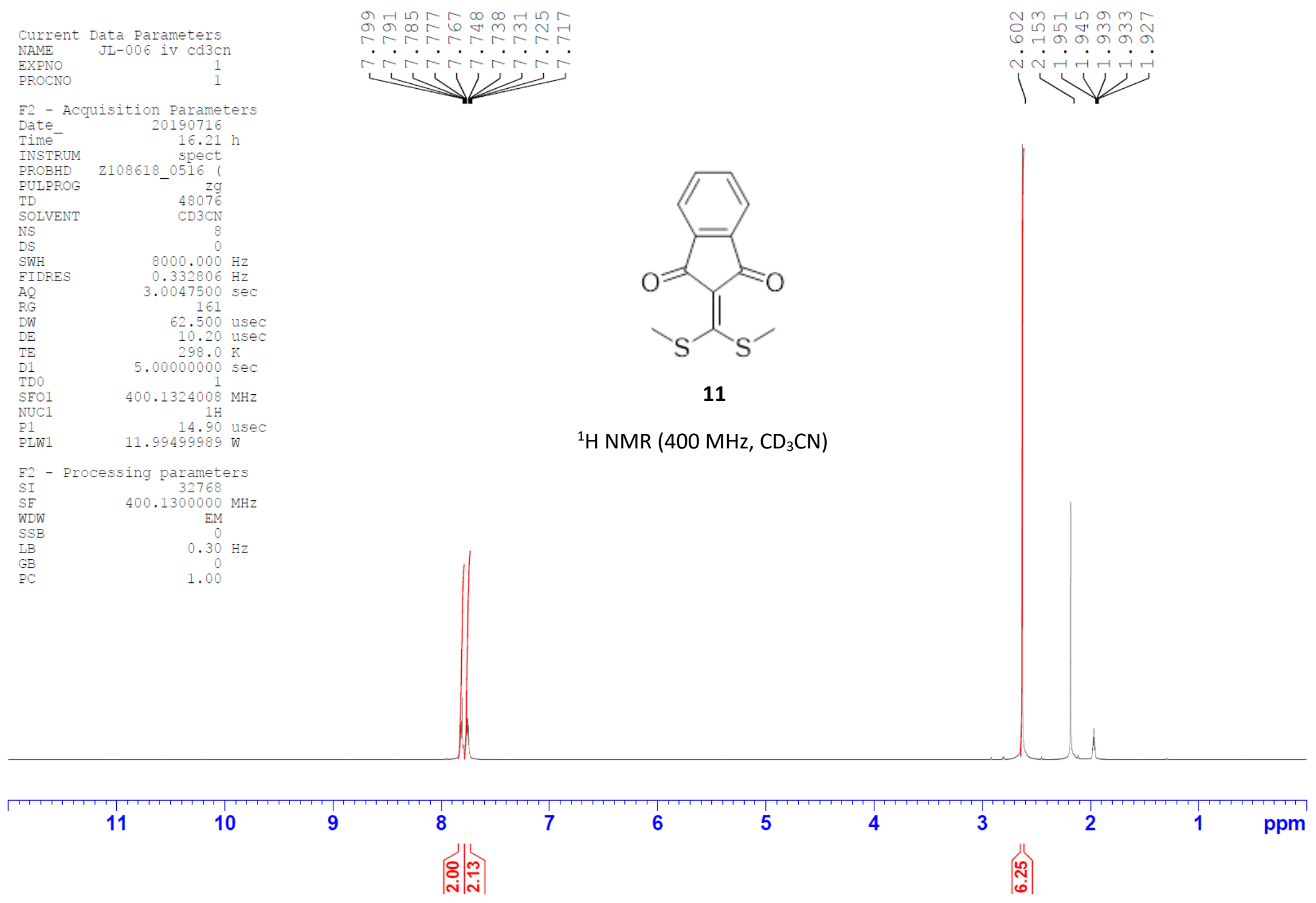


Current Data Parameters
NAME JL-006 iv cd3cn

EXPNO

F2 - Acquisition Parameters

Date_ 20190716

INSTRUM spect

$\begin{array}{ll}\text { PROBHD } & \text { 2108618_0516 } \\ \text { PULPROG } & \end{array}$

TD

NS

SWH

$A Q$
$R G$

$\mathrm{RG}$
$\mathrm{DW}$
$\mathrm{DE}$

$2.00000000 \mathrm{~K}$

$2.00000000 \mathrm{sec}$

$100.6228303 \mathrm{MHz}$

$13 \mathrm{C}$

$400.1316005 \mathrm{MHz}$

waltz16

$\begin{array}{lr}\text { PLW2 } & 11.99499989 \mathrm{~W} \\ \text { PLW12 } & 0.32877001 \mathrm{~W} \\ \text { PLW13 } & 0.16537000 \mathrm{~W}\end{array}$

F2 - Processing parameters

$\begin{array}{ll}\text { SI } & 65536 \\ \text { SF } & 100.6126708 \mathrm{MHz}\end{array}$

$\begin{array}{lc}\text { SF } & 100.6126708 \mathrm{MHz} \\ \text { WDW } & \text { EM }\end{array}$

$\begin{array}{lll}\text { SSB } & 0 & 1.00 \mathrm{~Hz}\end{array}$

PC 1.40

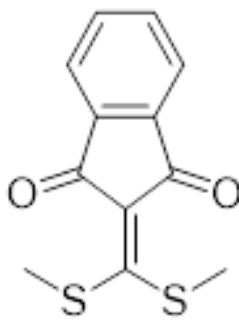

11

${ }^{13} \mathrm{C}\left\{{ }^{1} \mathrm{H}\right\}$ NMR $\left(100 \mathrm{MHz}, \mathrm{CD}_{3} \mathrm{CN}\right)$ 


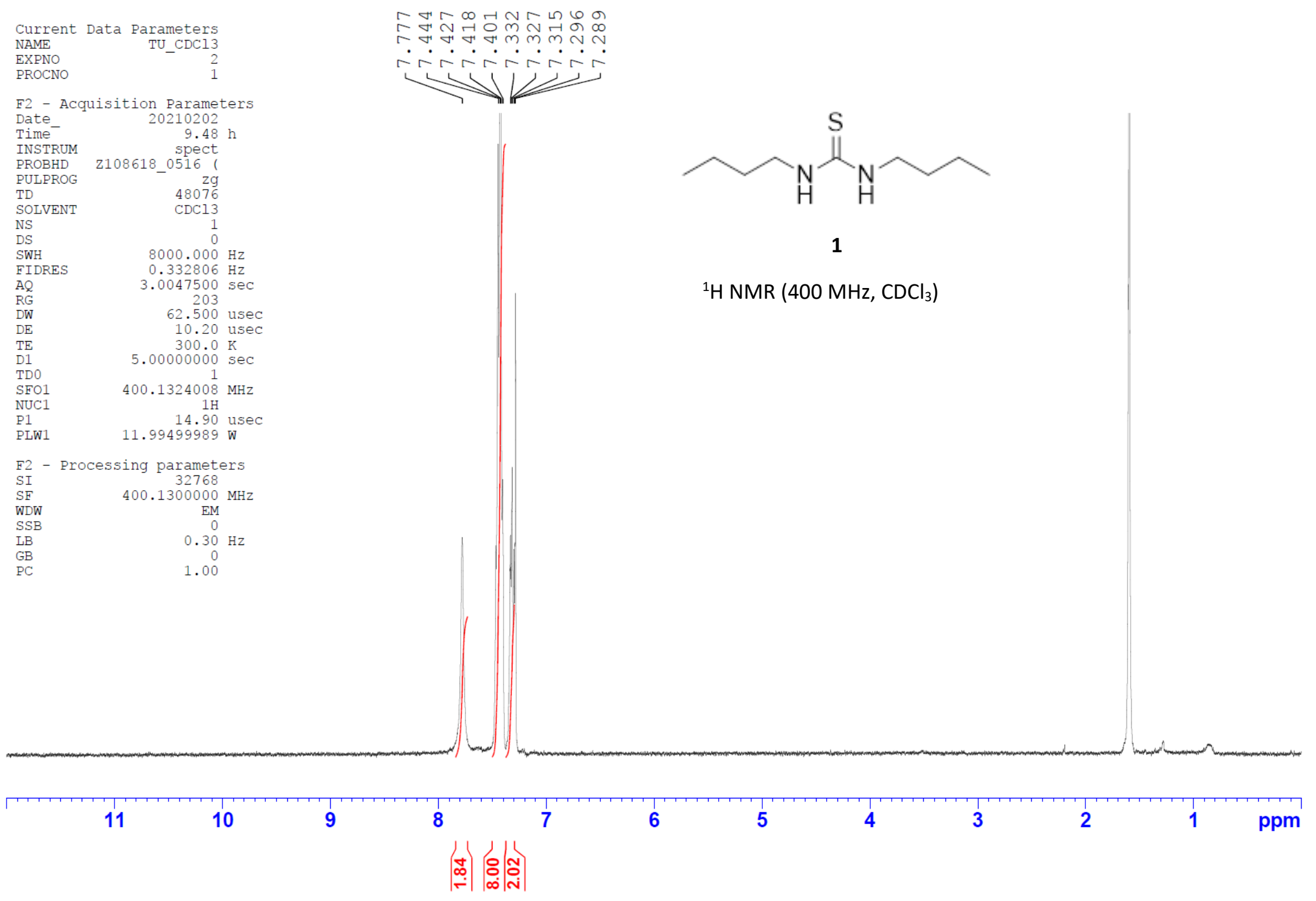




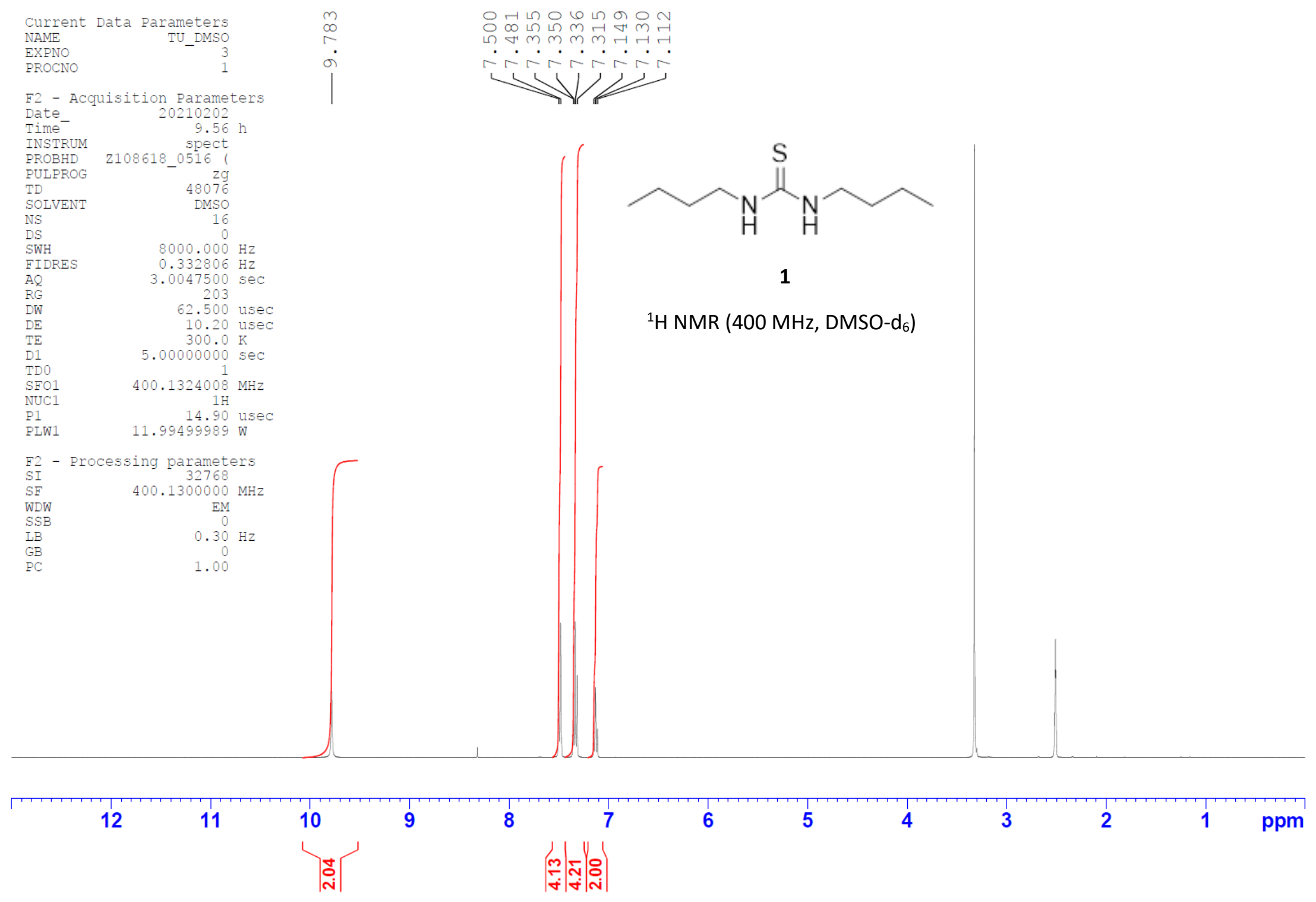




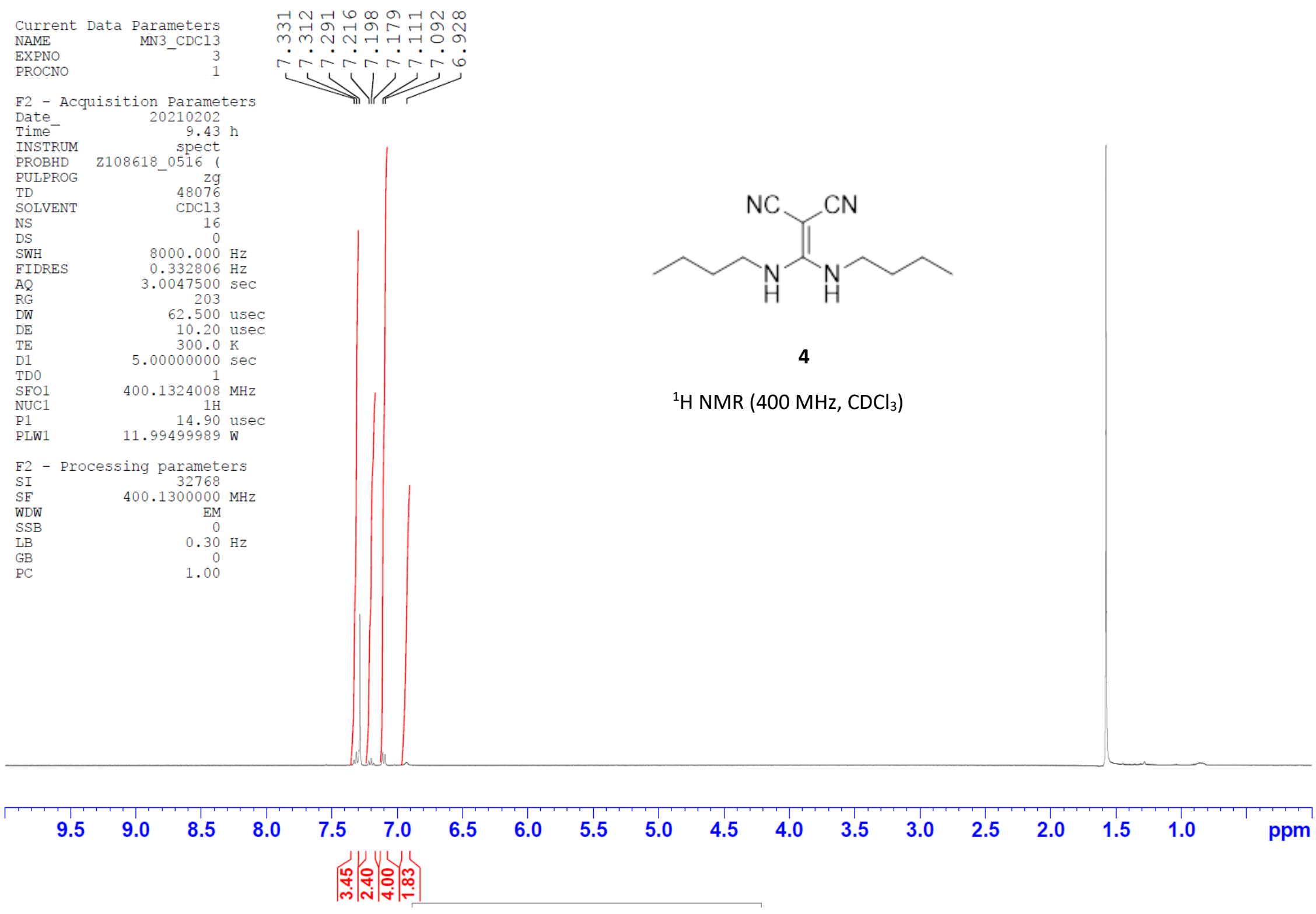




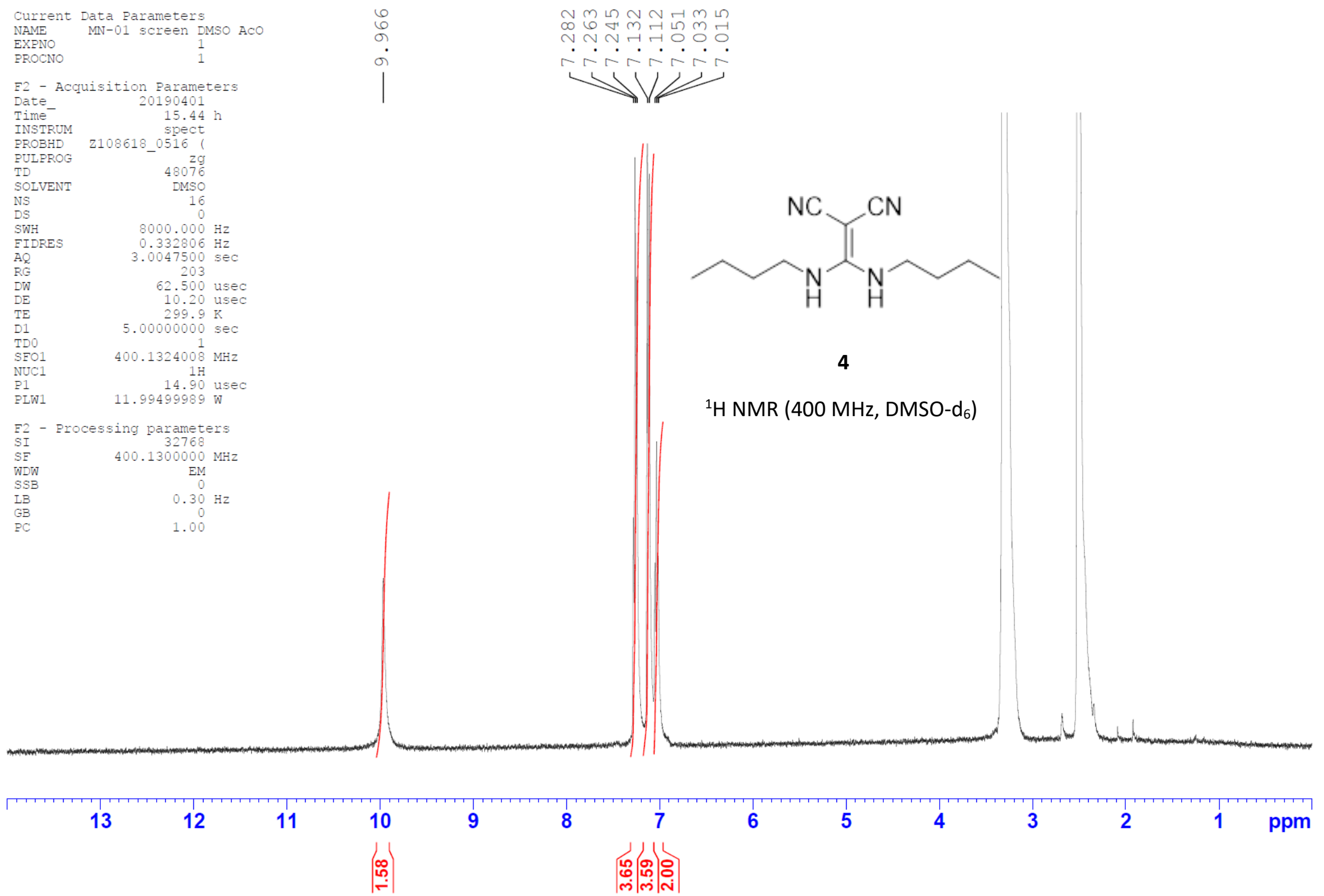




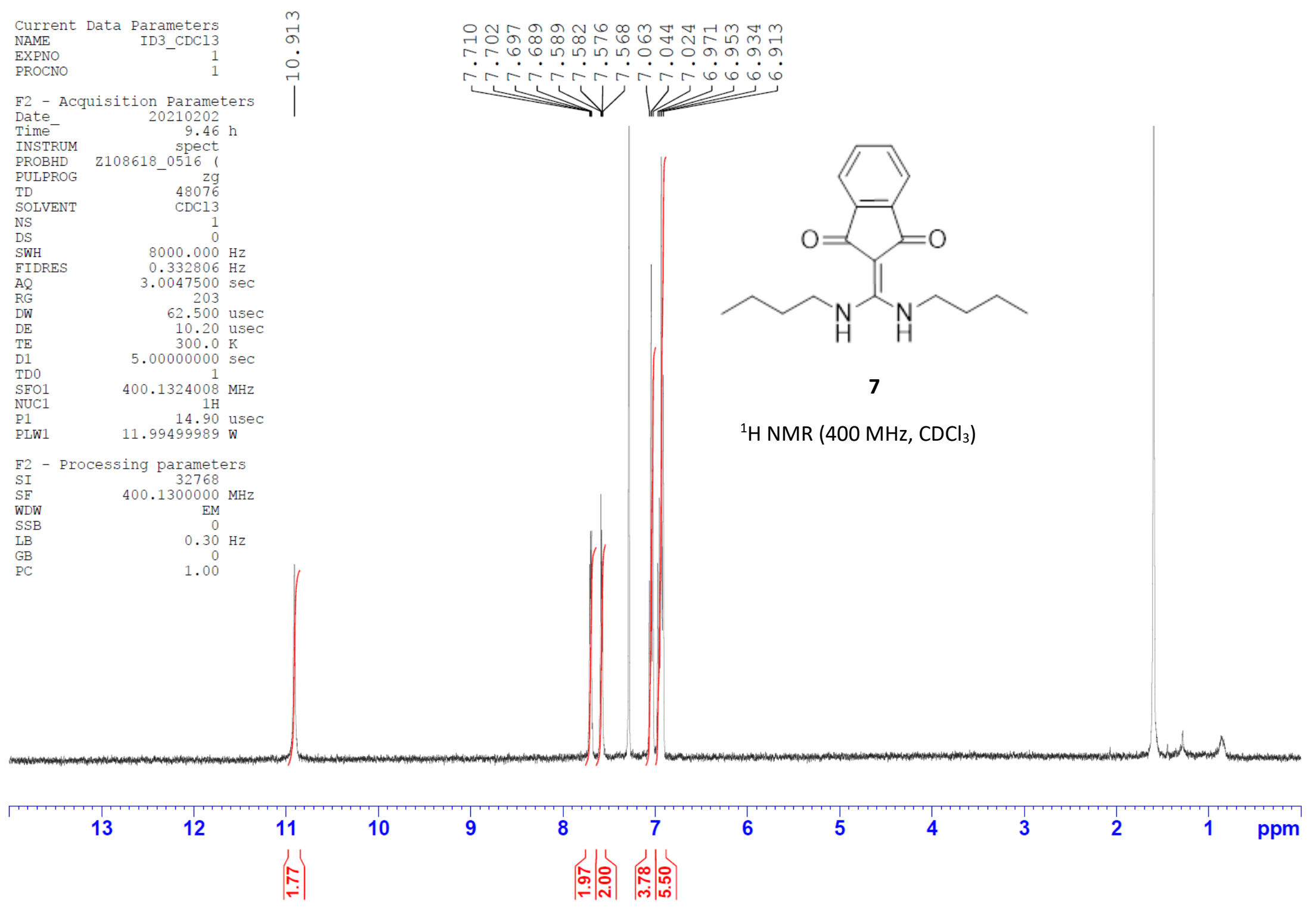




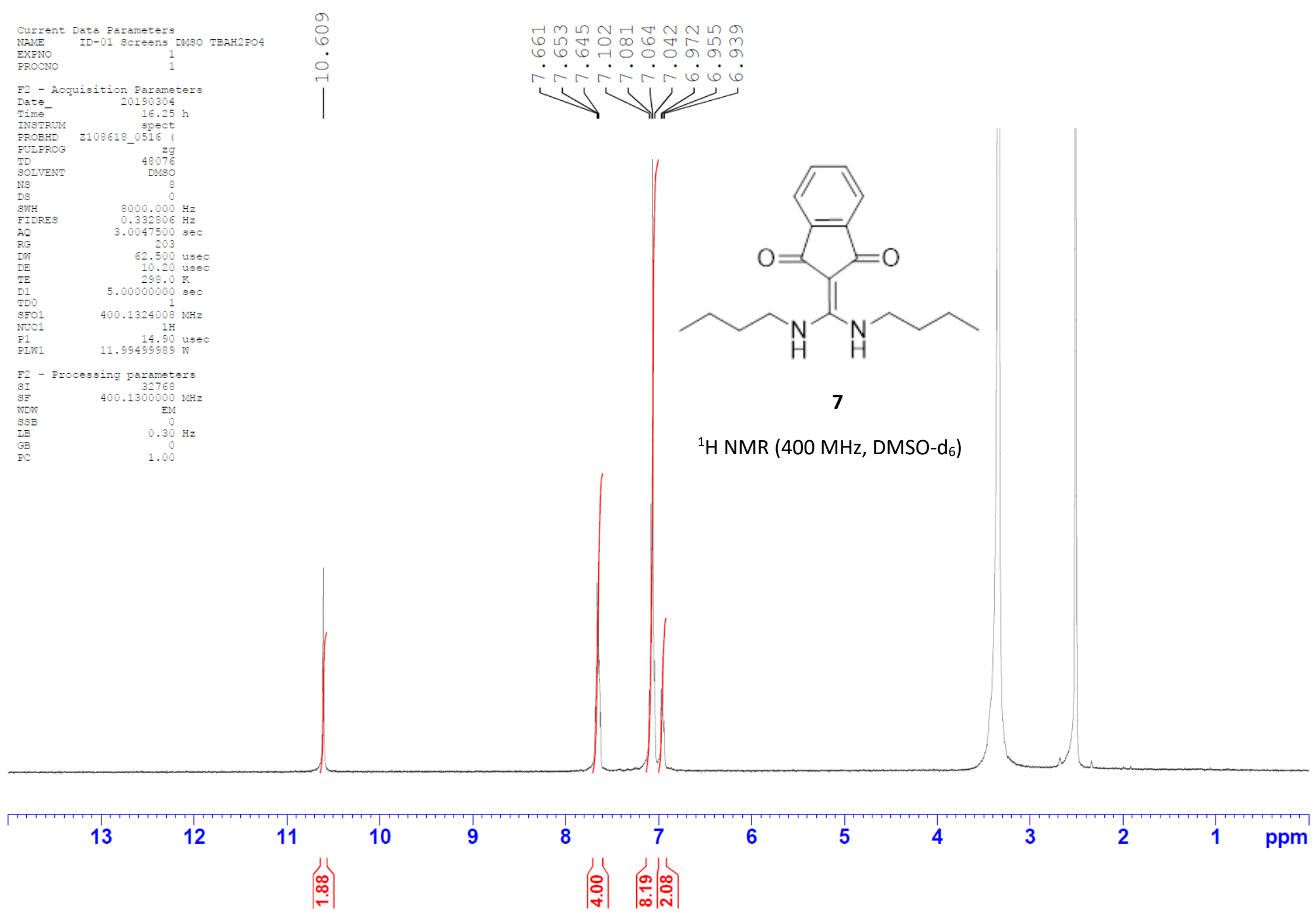


${ }^{1} \mathrm{H}$ NMR anion screens

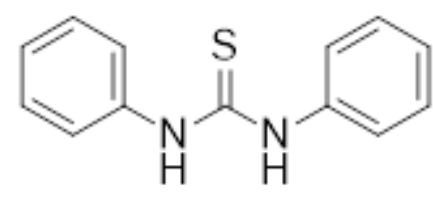

$\mathrm{TsO}$

$\mathrm{NO}_{3}^{-}$

$\mathrm{AcO}$

$\mathrm{HSO}_{4}^{-}$

$\mathrm{H}_{2} \mathrm{PO}_{4}^{-}$

$\mathrm{F}^{-}$

$\mathrm{Br}^{-}$

$\mathrm{Cl}^{-}$

No anion

Fig. S1. Anion screen compound 3. ${ }^{1} \mathrm{H} \mathrm{NMR}\left(400 \mathrm{MHz}, \mathrm{CD}_{3} \mathrm{CN}\right)$. 


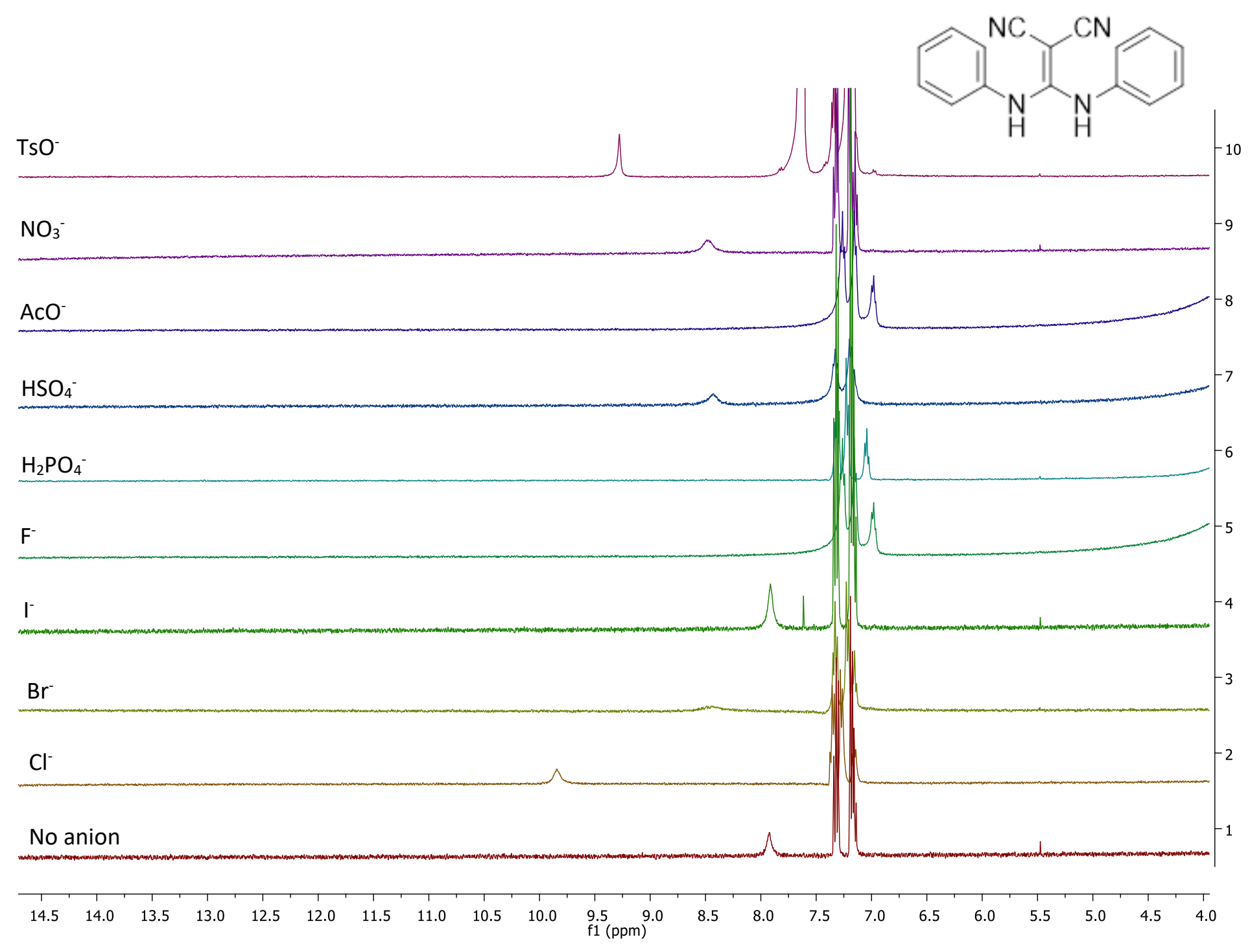

Fig. S2. Anion screen compound 6. ${ }^{1} \mathrm{H} \mathrm{NMR}\left(400 \mathrm{MHz}, \mathrm{CD}_{3} \mathrm{CN}\right)$. 




Fig. S3. Anion screen compound 9. ${ }^{1} \mathrm{H} \mathrm{NMR}\left(400 \mathrm{MHz}, \mathrm{CD}_{3} \mathrm{CN}\right)$. 


\section{${ }^{1} \mathrm{H}$ NMR titration data}
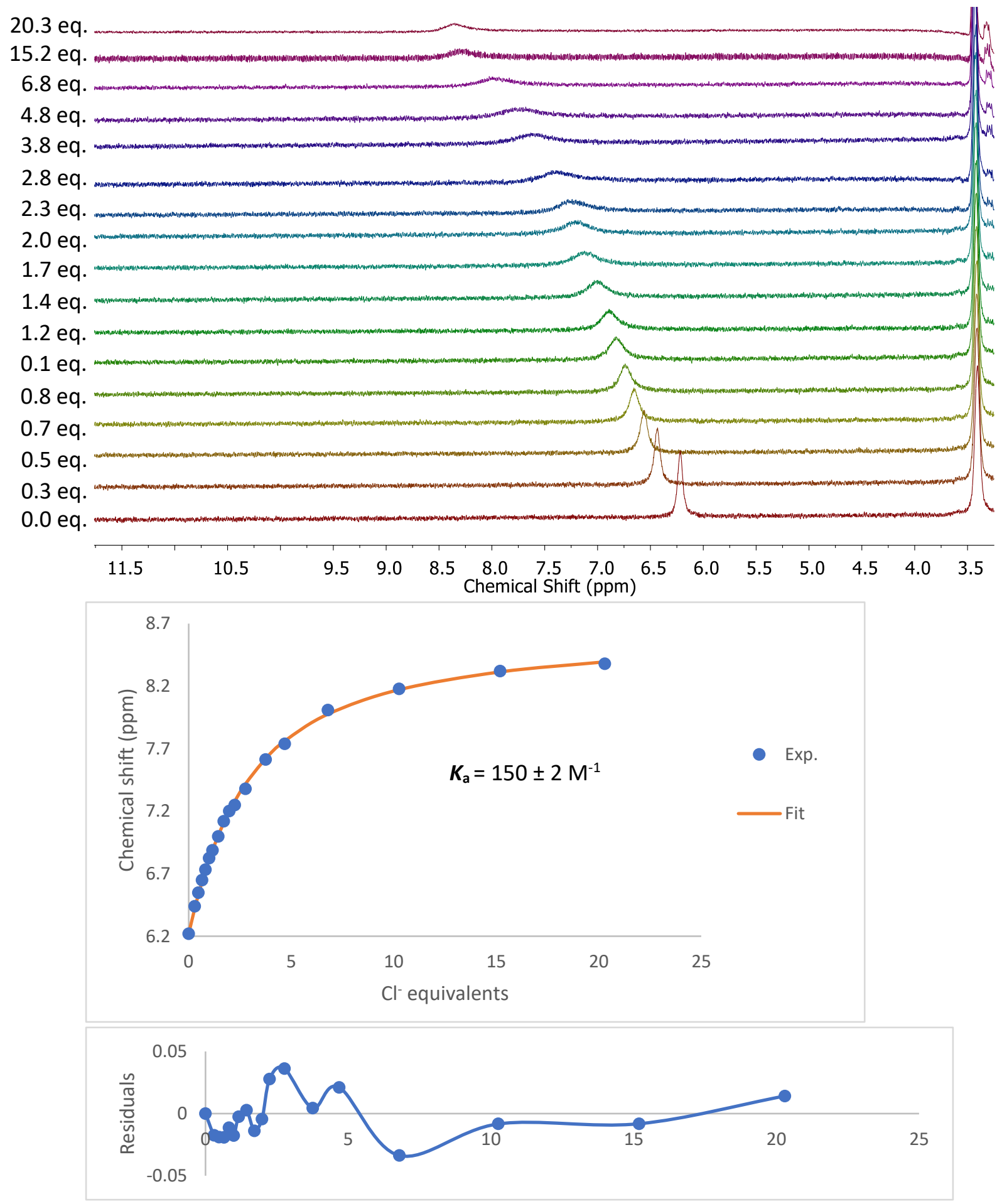

Fig. S4. ${ }^{1} \mathrm{H}$ NMR (400 MHz, $\left.\mathrm{CD}_{3} \mathrm{CN}\right)$ stack plot of $1(2.9 \mathrm{mM})$ TBACl at $300 \mathrm{~K}, 1: 1$ binding fit, residuals. Average $K_{a}$ of two titrations and fitting error shown. 
19.5 eq.

14.8 eq.

$9.1 \mathrm{eq}$.

6.2 eq.

4.5 eq.

3.7 eq.

2.7 eq.

2.2 eq.

$1.9 \mathrm{eq}$.

1.6 eq.

1.3 eq.

1.1 eq.

0.9 eq.

0.7 eq.

0.6 eq.

0.3 eq.

0.0 eq.
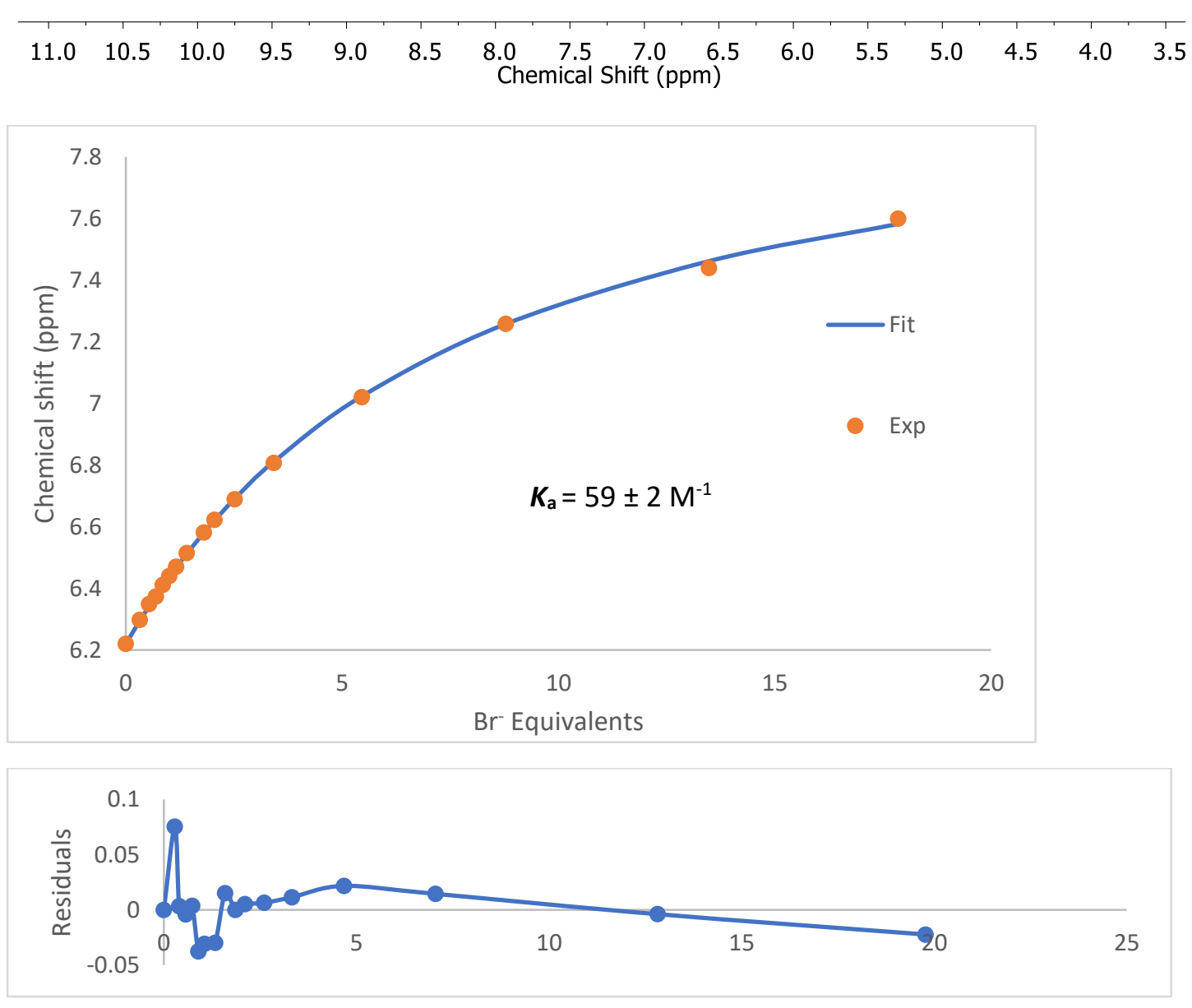

Fig. S5. ${ }^{1} \mathrm{H}$ NMR $\left(400 \mathrm{MHz}, \mathrm{CD}_{3} \mathrm{CN}\right)$ stack plot of $1(2.8 \mathrm{mM}) \mathrm{TBABr}$ at $300 \mathrm{~K}, 1: 1$ binding fit, residuals. Average $\mathrm{K}_{\mathrm{a}}$ of two titrations and fitting error shown. 

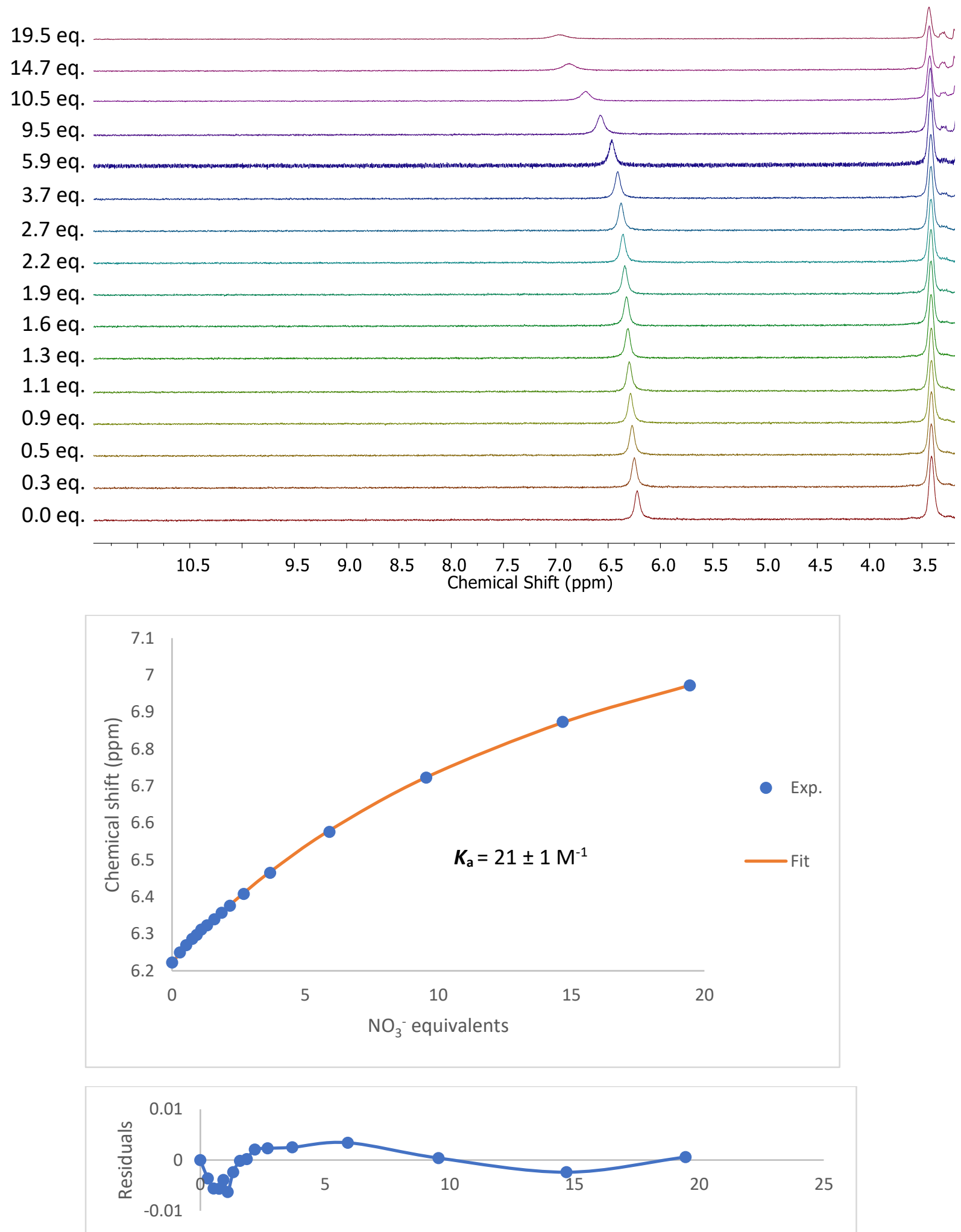

Fig S6. ${ }^{1} \mathrm{H}$ NMR $\left(400 \mathrm{MHz}, \mathrm{CD}_{3} \mathrm{CN}\right)$ stack plot of $1(2.8 \mathrm{mM}) \mathrm{TBANO}_{3}$ at $300 \mathrm{~K} .1: 1$ binding fit, residuals. Average $\mathrm{K}_{\mathrm{a}}$ of two titrations and fitting error shown. 
19.5 eq.

15.3 eq.

12.6 eq.

9.0 eq.

6.9 eq.

4.5 eq.

3.4 eq.

2.8 eq.

1.9 eq.

$1.6 \mathrm{eq}$.

1.3 eq.

1.0 eq.

0.8 eq.

0.6 eq.

0.4 eq.

0.0 eq.
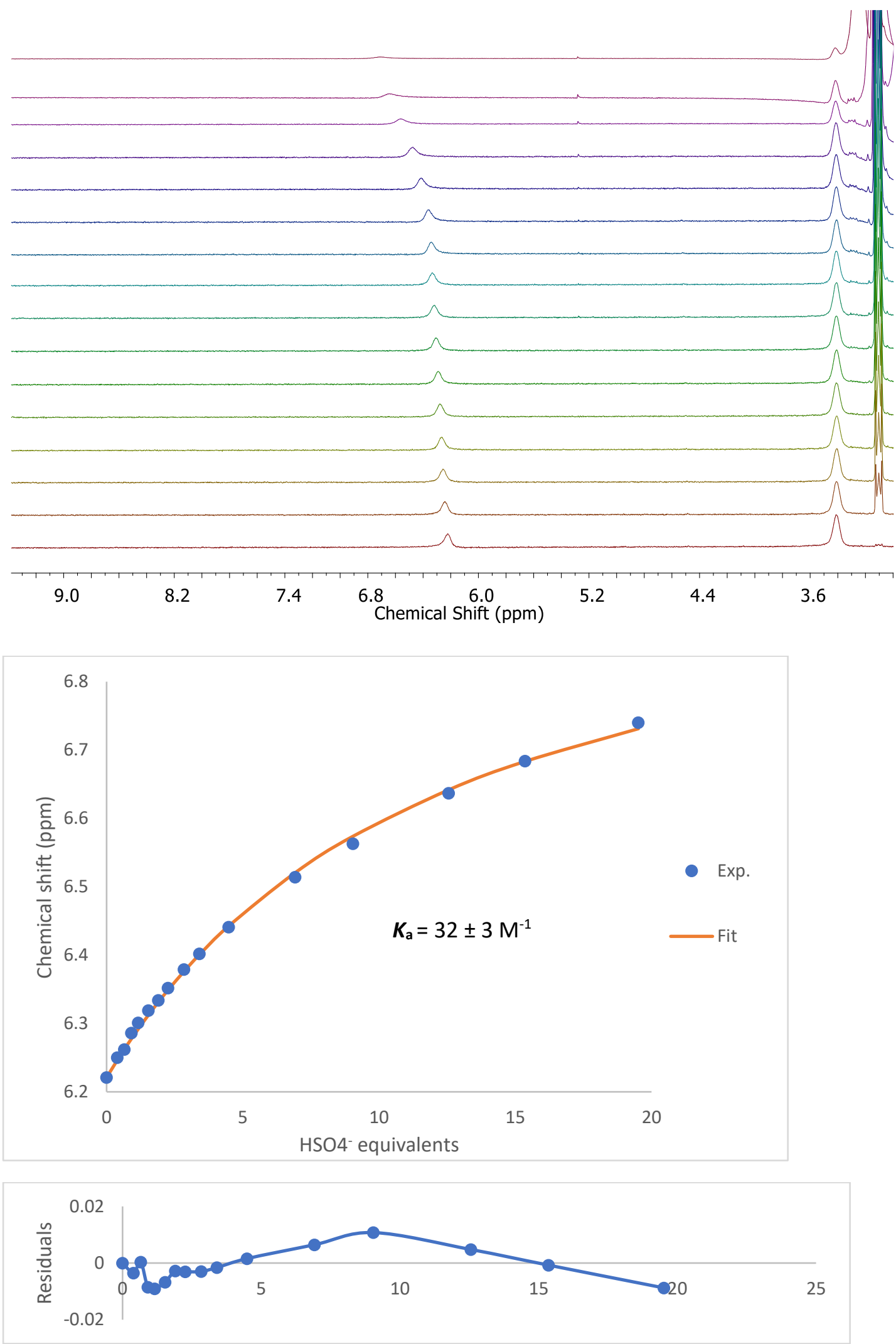

Fig. S7. ${ }^{1} \mathrm{H}$ NMR ( $400 \mathrm{MHz}, \mathrm{CD}_{3} \mathrm{CN}$ ) stack plot of 1 (2.8 mM) $\mathrm{TBAHSO}_{4}$ at $300 \mathrm{~K}$, binding fit, residuals. Average $\mathrm{K}_{\mathrm{a}}$ of two titrations and fitting error shown. 

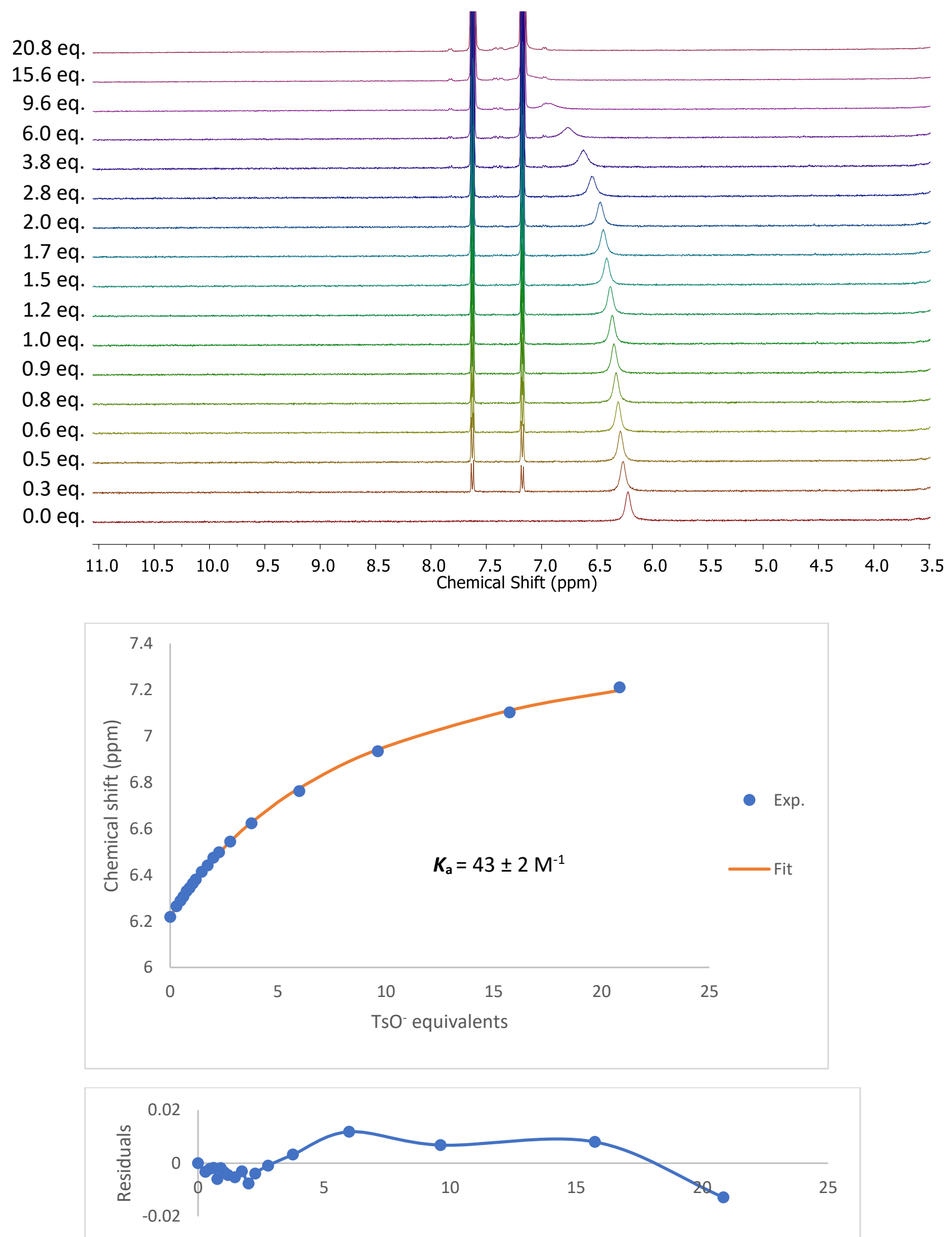

Fig. S8. ${ }^{1} \mathrm{H}$ NMR (400 MHz, $\left.\mathrm{CD}_{3} \mathrm{CN}\right)$ stack plot of 1 ( $2.8 \mathrm{mM}$ ) TBAOTs at $300 \mathrm{~K}, 1: 1$ binding fit, residuals. Average $\mathrm{K}_{\mathrm{a}}$ of two titrations and fitting error shown. 

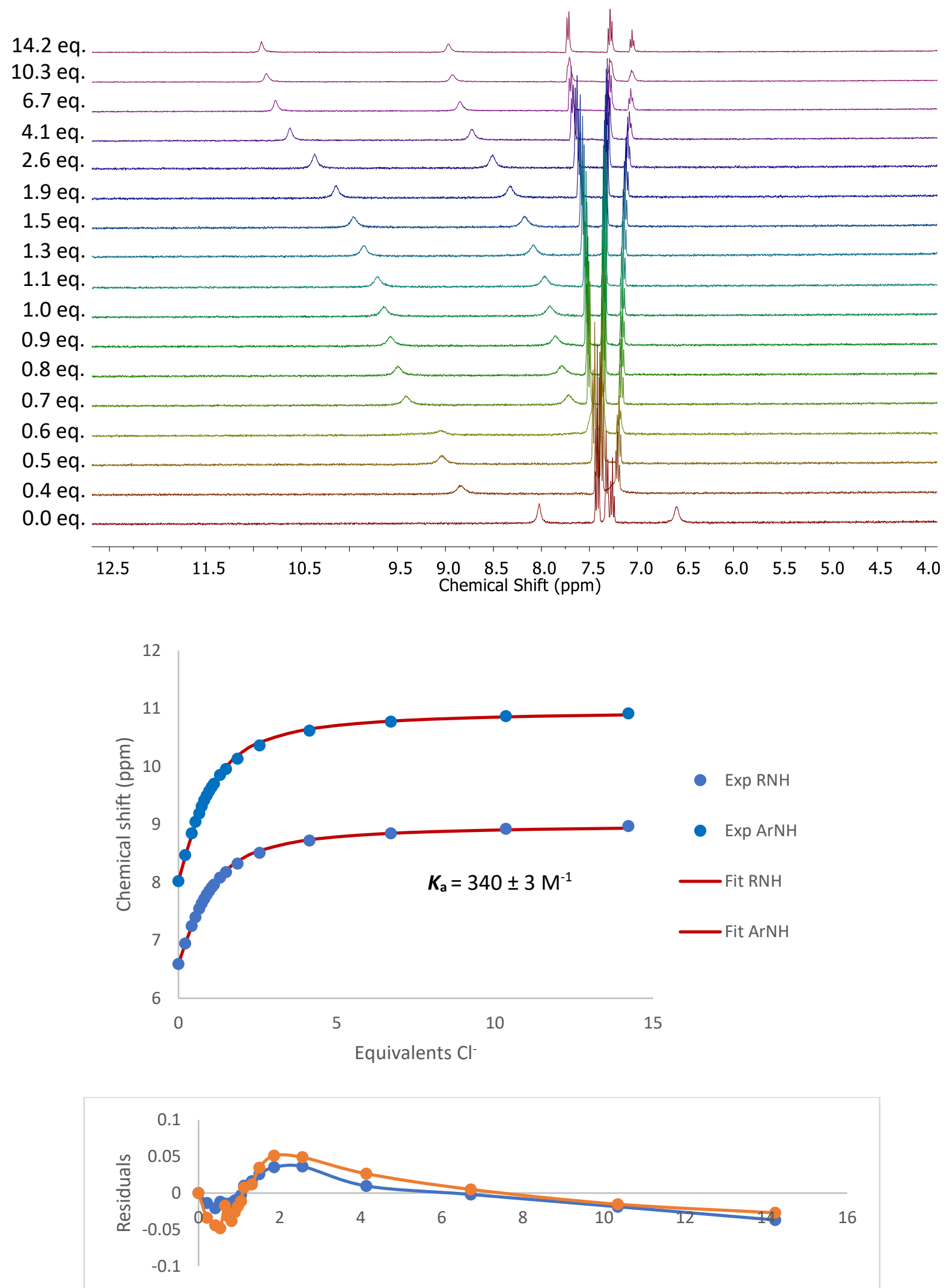

Fig. S9. ${ }^{1} \mathrm{H}$ NMR $\left(400 \mathrm{MHz}, \mathrm{CD}_{3} \mathrm{CN}\right)$ stack plot of $2(3.6 \mathrm{mM})$ TBACl at $300 \mathrm{~K}, 1: 1$ binding fit, residuals. Average $\mathrm{K}_{\mathrm{a}}$ of two titrations and fitting error shown. 

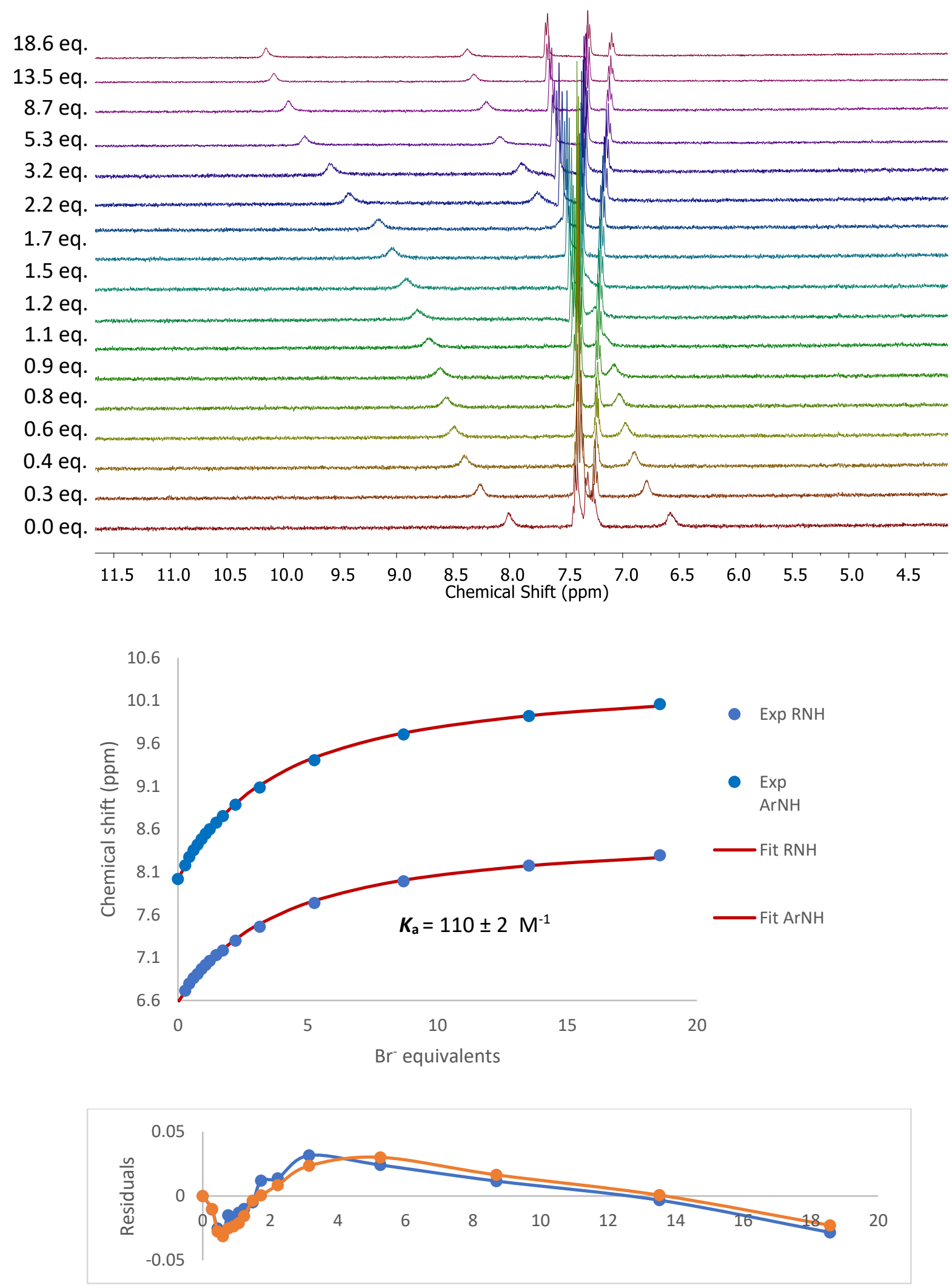

Fig. S10. ${ }^{1} \mathrm{H}$ NMR $\left(400 \mathrm{MHz}, \mathrm{CD}_{3} \mathrm{CN}\right)$ stack plot of $2(2.9 \mathrm{mM}) \mathrm{TBABr}$ at $300 \mathrm{~K}, 1: 1$ binding fit, residuals. Average $\mathrm{K}_{\mathrm{a}}$ of two titrations and fitting error shown. 

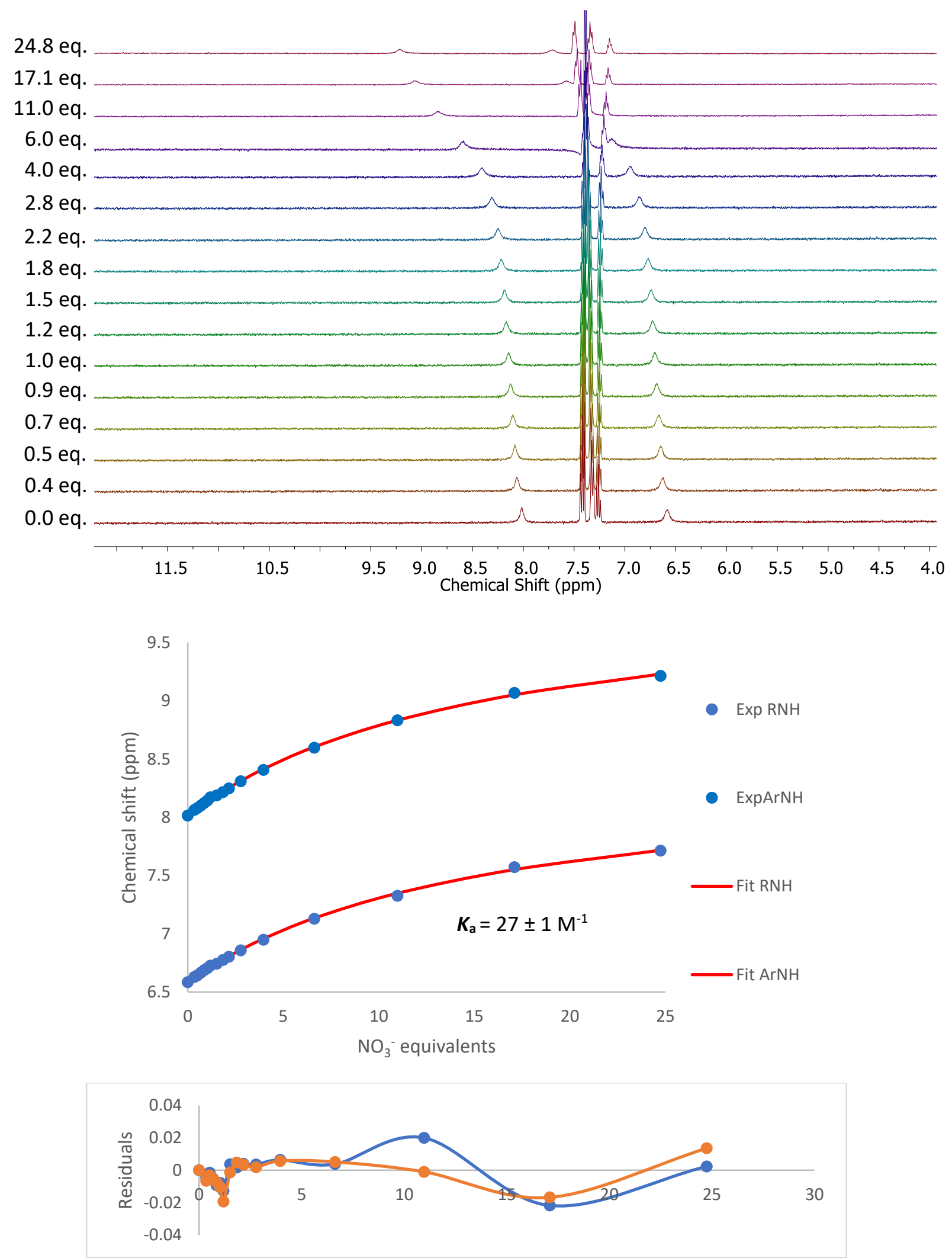

Fig. S11. ${ }^{1} \mathrm{H} \mathrm{NMR}\left(400 \mathrm{MHz}, \mathrm{CD}_{3} \mathrm{CN}\right)$ stack plot of $2(2.5 \mathrm{mM}) \mathrm{TBANO}_{3}$ at $300 \mathrm{~K}, 1: 1$ binding fit, residuals. Average $\mathrm{K}_{\mathrm{a}}$ of two titrations and fitting error shown. 

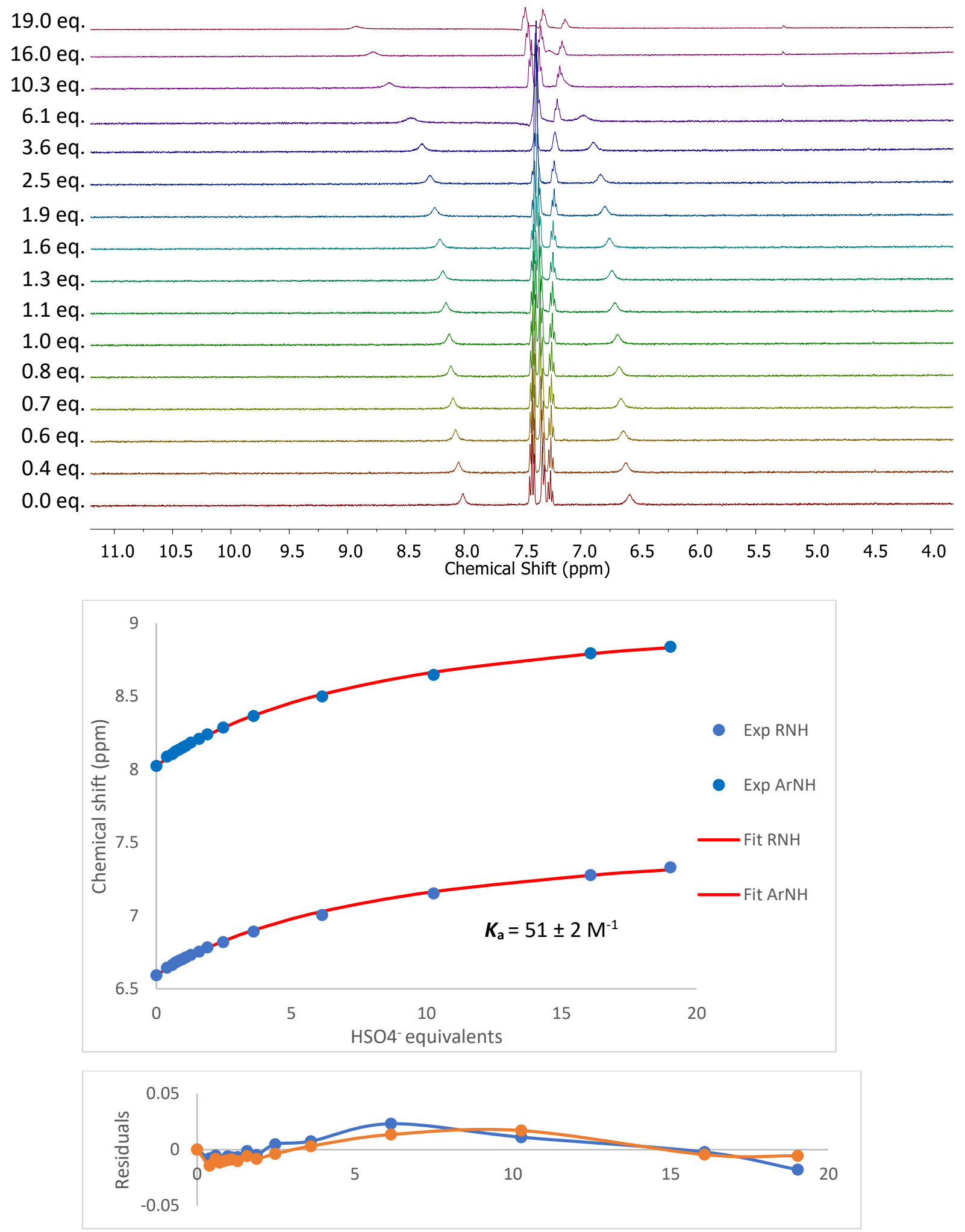

Fig. S12. ${ }^{1} \mathrm{H}$ NMR $\left(400 \mathrm{MHz}, \mathrm{CD}_{3} \mathrm{CN}\right)$ stack plot of $2(2.5 \mathrm{mM})$ with $\mathrm{TBAHSO}_{4}$ at $300 \mathrm{~K}, 1: 1$ binding fit, residuals. Average $\mathrm{K}_{\mathrm{a}}$ of two titrations and fitting error shown. 

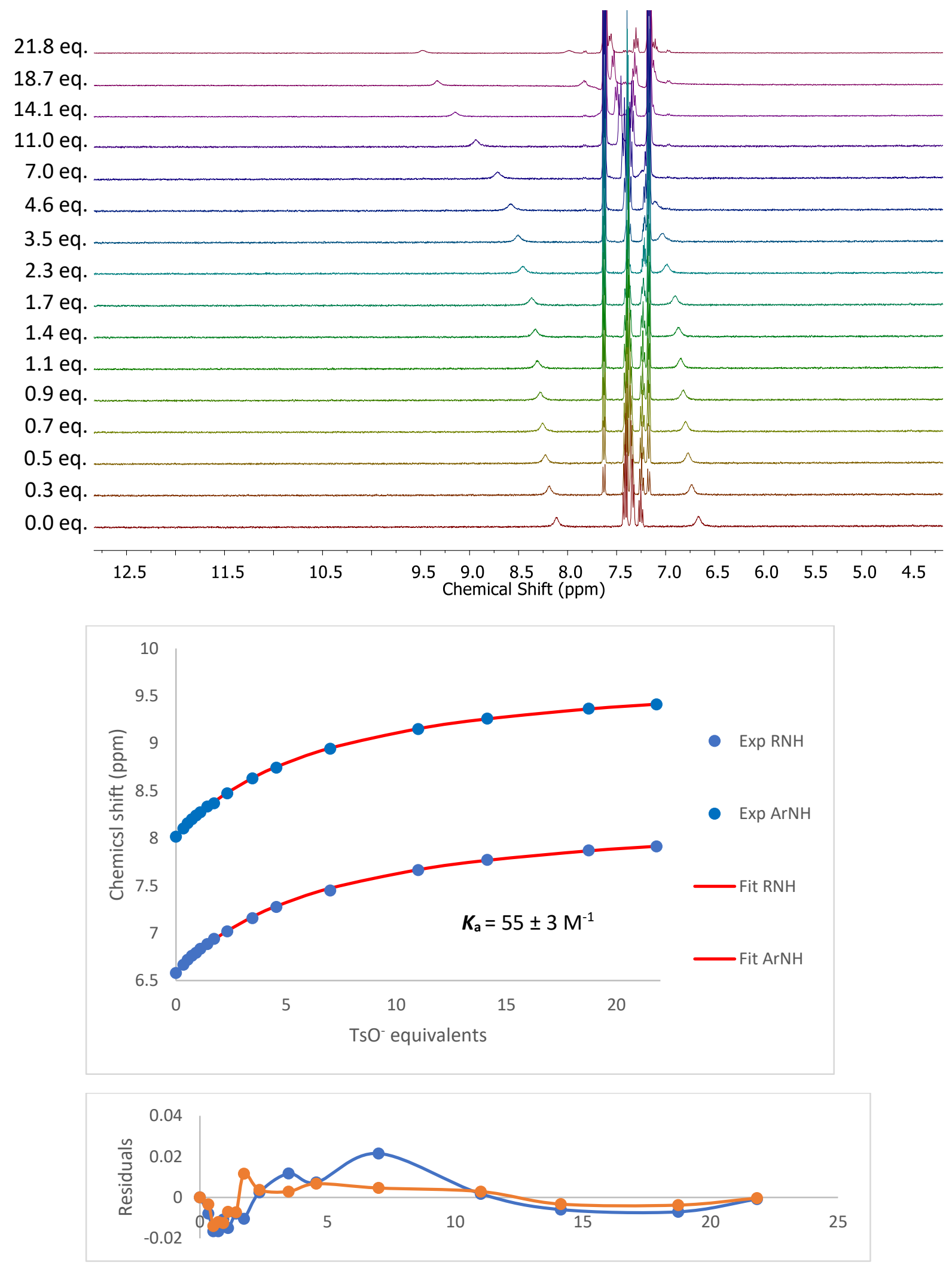

Fig. S13. ${ }^{1} \mathrm{H}$ NMR ( $400 \mathrm{MHz}, \mathrm{CD}_{3} \mathrm{CN}$ ) stack plot of 2 (2.5 mM) TBAOTs at $300 \mathrm{~K}, 1: 1$ binding fit, residuals. Average $\mathrm{K}_{\mathrm{a}}$ of two titrations and fitting error shown. 

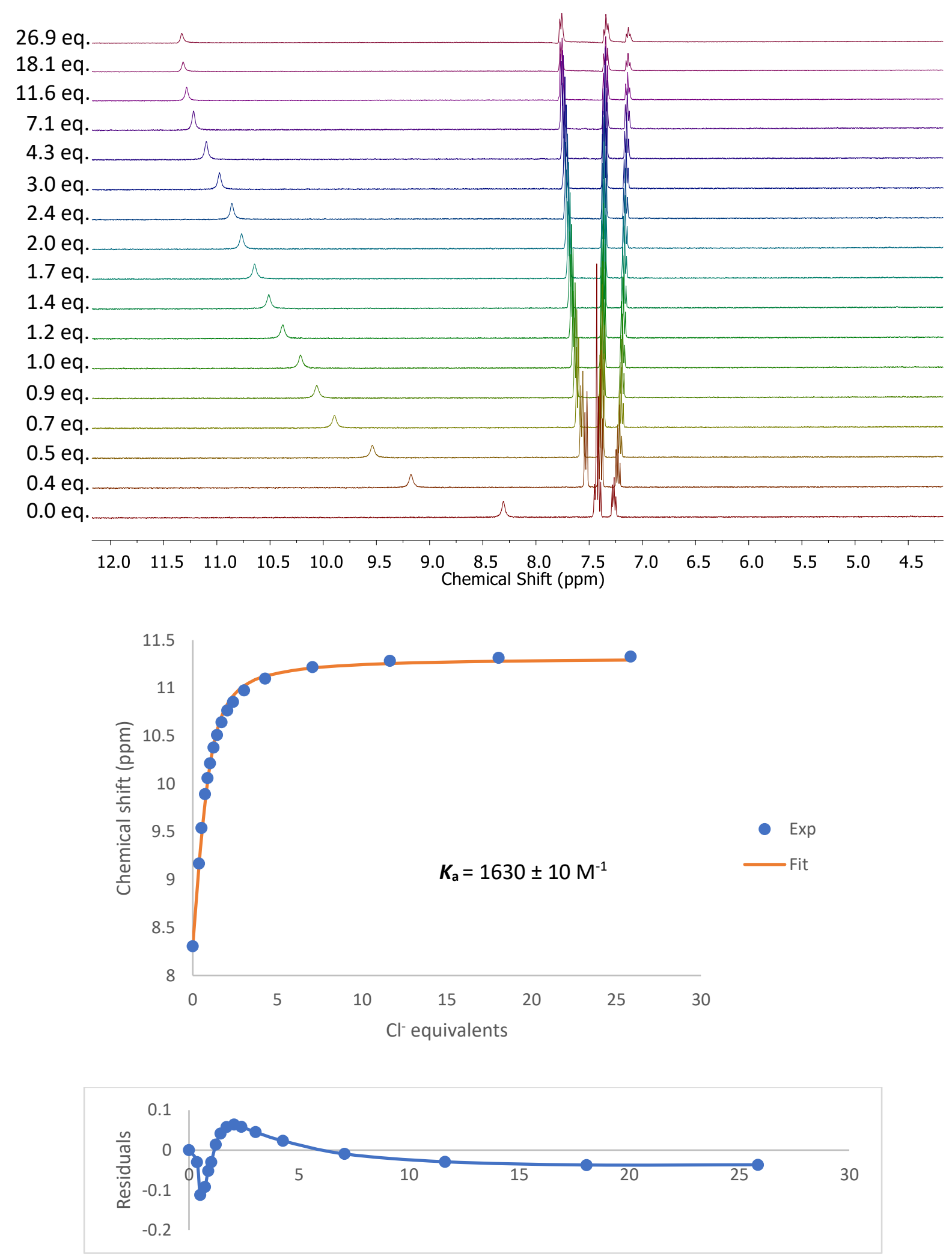

Fig. S14. ${ }^{1} \mathrm{H}$ NMR ( $400 \mathrm{MHz}, \mathrm{CD}_{3} \mathrm{CN}$ ) stack plot of 3 (2.6 mM) TBACl at $300 \mathrm{~K}, 1: 1$ binding fit, residuals. Average $\mathrm{K}_{\mathrm{a}}$ of two titrations and fitting error shown. 

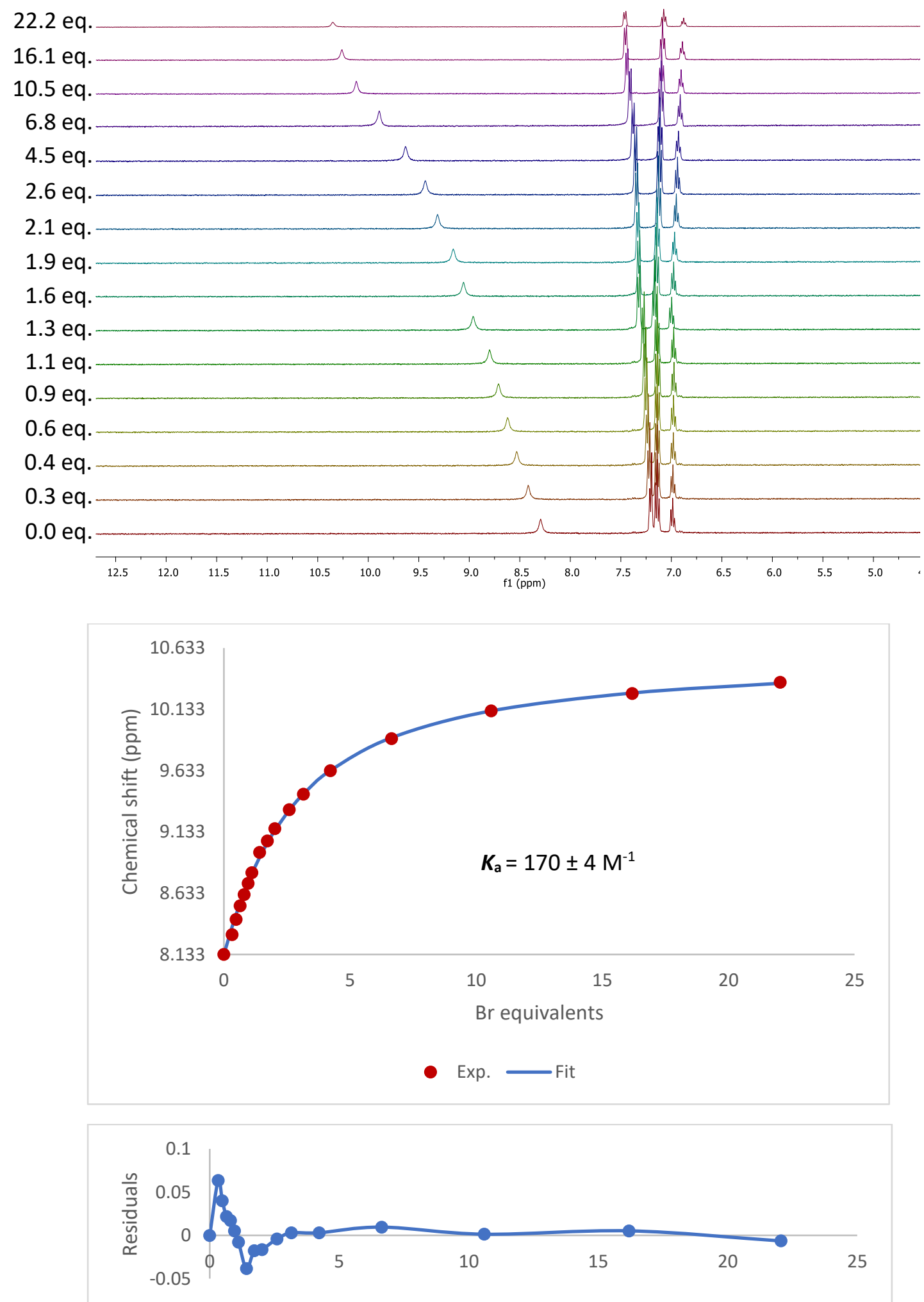

Fig. S15. ${ }^{1} \mathrm{H}$ NMR $\left(400 \mathrm{MHz}, \mathrm{CD}_{3} \mathrm{CN}\right)$ stack plot of $3(2.5 \mathrm{mM}) \mathrm{TBABr}$ in at $300 \mathrm{~K}, 1: 1$ binding fit, residuals. Average $\mathrm{K}_{\mathrm{a}}$ of two titrations and fitting error shown. 

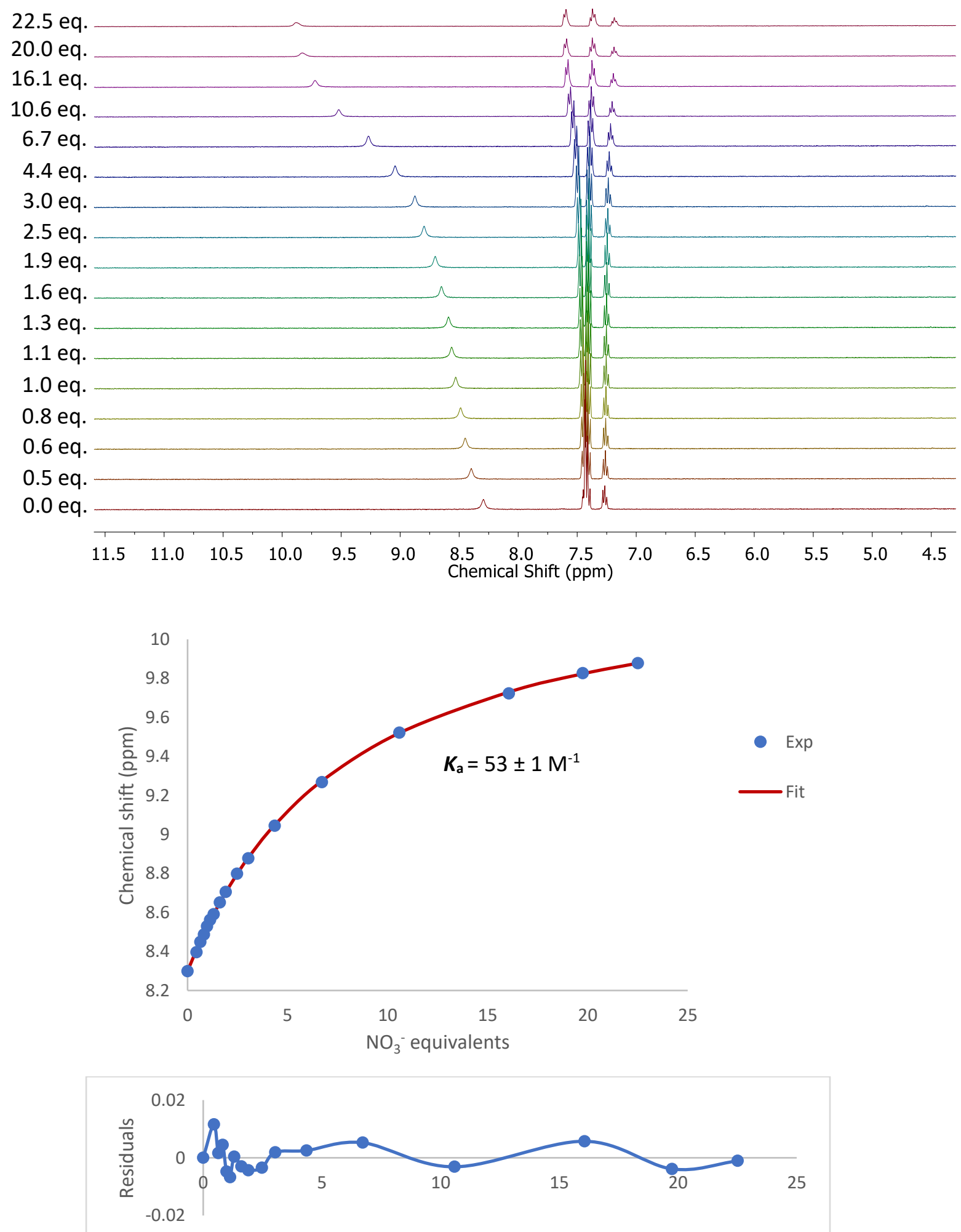

Fig. S16. ${ }^{1} \mathrm{H}$ NMR ( $400 \mathrm{MHz}, \mathrm{CD}_{3} \mathrm{CN}$ ) stack plot of $3\left(2.6 \mathrm{mM}\right.$ ) $\mathrm{TBANO}_{3}$ in at $300 \mathrm{~K}, 1: 1$ binding fit, residuals. Average $\mathrm{K}_{\mathrm{a}}$ of two titrations and fitting error shown. 

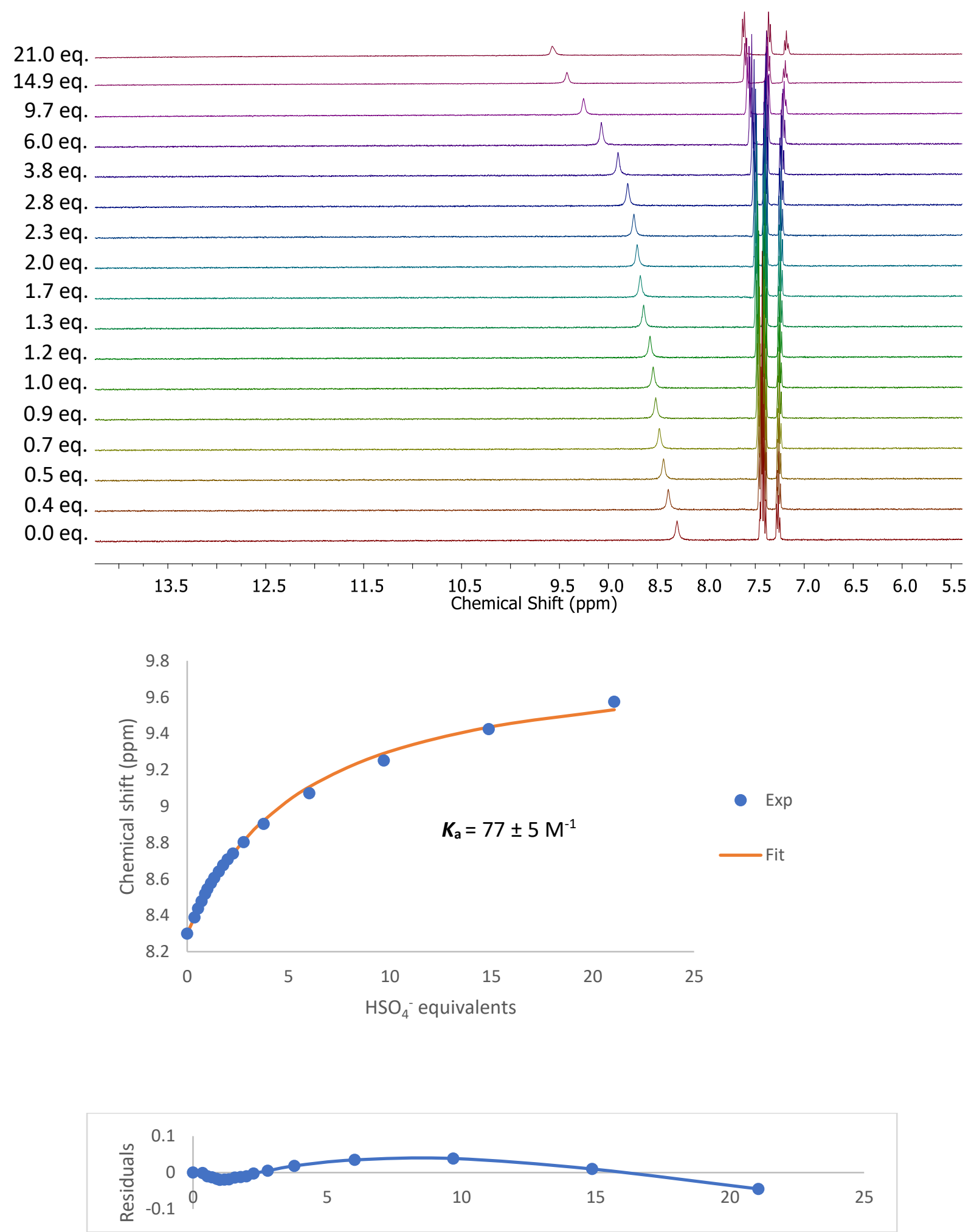

Fig. S17. ${ }^{1} \mathrm{H}$ NMR $\left(400 \mathrm{MHz}, \mathrm{CD}_{3} \mathrm{CN}\right)$ stack plot of $3(2.6 \mathrm{mM}) \mathrm{TBAHSO}_{4}$ at $300 \mathrm{~K}, 1: 1$ binding fit, residuals. Average $\mathrm{K}_{\mathrm{a}}$ of two titrations and fitting error shown. 

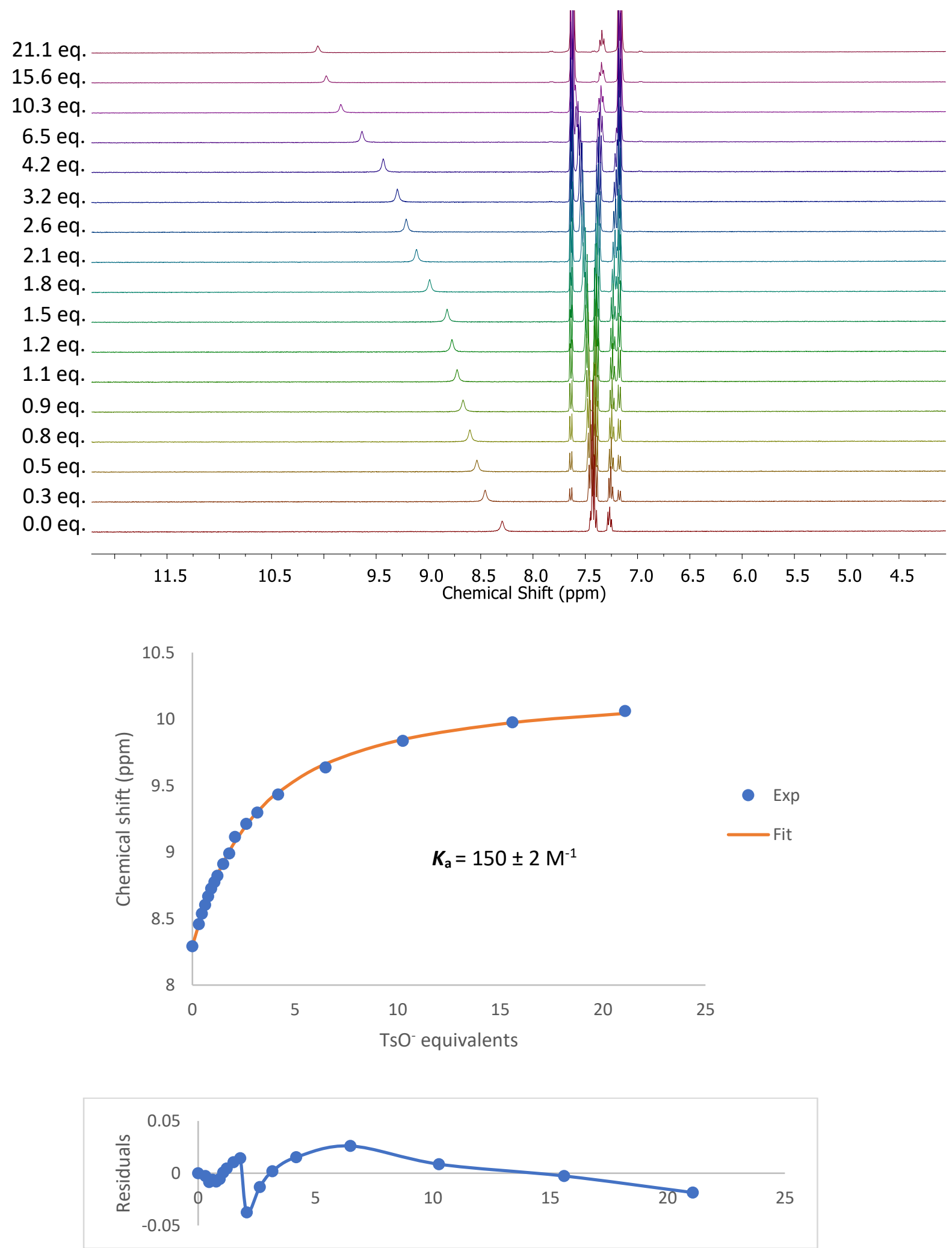

Fig. S18. ${ }^{1} \mathrm{H}$ NMR $\left(400 \mathrm{MHz}, \mathrm{CD}_{3} \mathrm{CN}\right)$ stack plot of $3(2.7 \mathrm{mM})$ TBAOTs at $300 \mathrm{~K}, 1: 1$ binding fit, residuals. Average $K_{a}$ of two titrations and fitting error shown. 
24.8 eq.

18.5 eq.

11.0 eq.

7.1 eq.

4.9 eq.

3.5 eq.

2.0 eq.

$1.6 \mathrm{eq}$.

$1.4 \mathrm{eq}$.

1.2 eq.

1.0 eq.

0.8 eq.

0.7 eq.

0.6 eq.

0.4 eq.

0.3 eq.

0.0 eq.
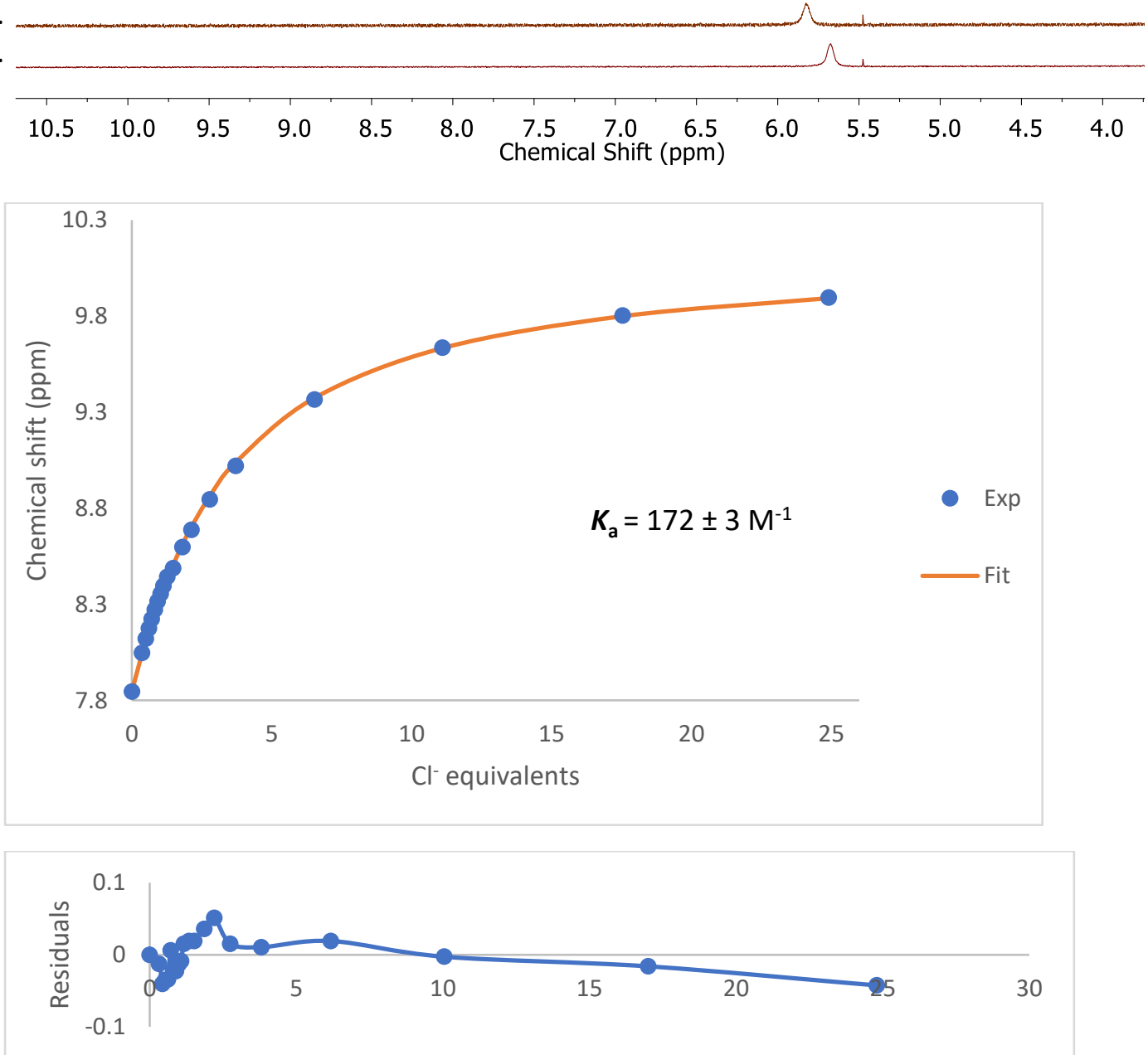

Fig. S19. ${ }^{1} \mathrm{H}$ NMR (400 MHz, $\left.\mathrm{CD}_{3} \mathrm{CN}\right)$ stack plot of 4 (3.1 mM) TBACl at $300 \mathrm{~K}, 1: 1$ binding fit, residuals. Average $\mathrm{K}_{\mathrm{a}}$ of two titrations and fitting error shown. 


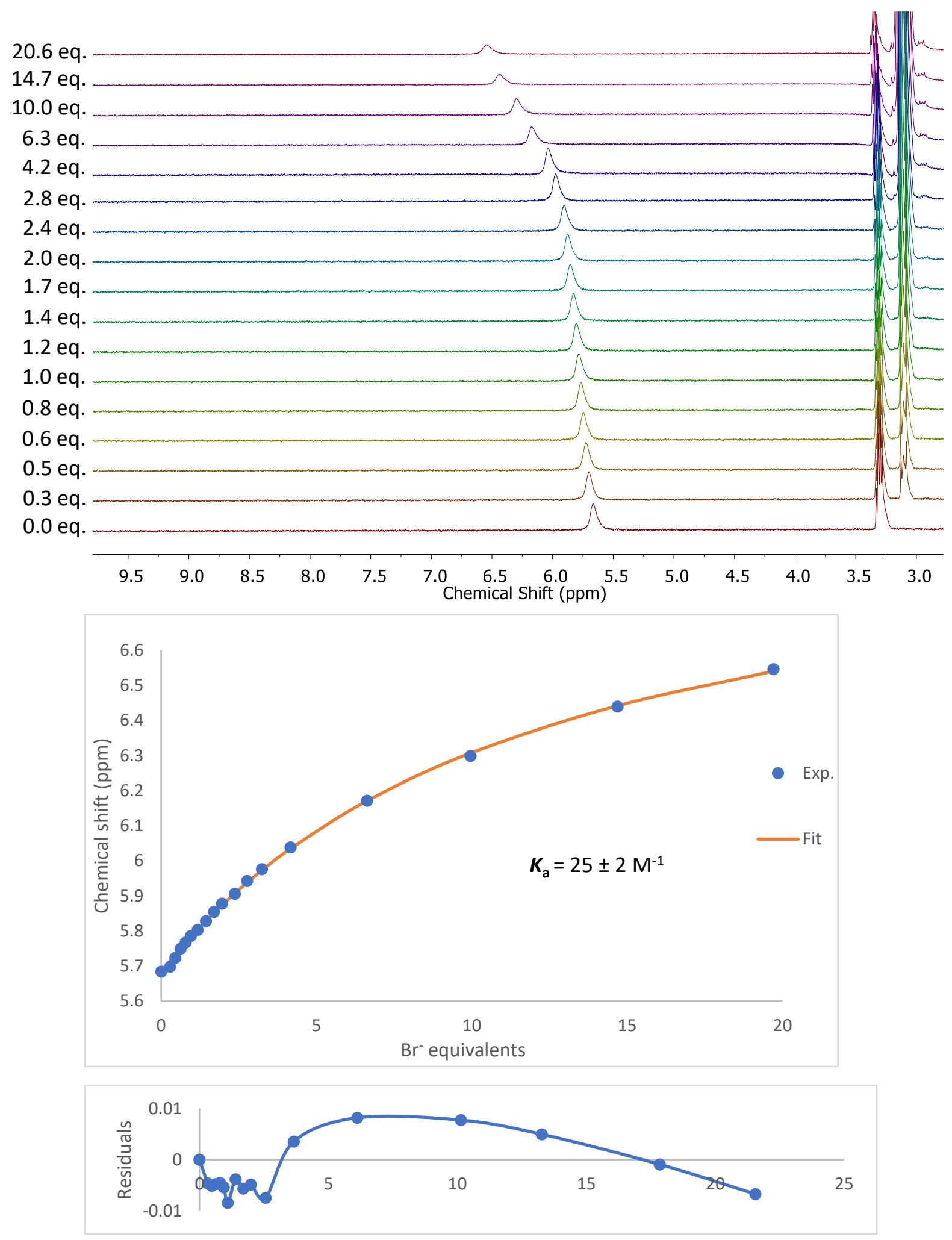

Fig. S20. ${ }^{1} \mathrm{H}$ NMR $\left(400 \mathrm{MHz}, \mathrm{CD}_{3} \mathrm{CN}\right)$ stack plot of $4(2.7 \mathrm{mM}) \mathrm{TBABr}$ at $300 \mathrm{~K}, 1: 1$ binding fit, residuals. Average $\mathrm{K}_{\mathrm{a}}$ of two titrations and fitting error shown. 
17.2 eq.

11.5 eq.

7.8 eq.

5.1 eq.

3.4 eq.

2.0 eq.

1.8 eq.

$1.6 \mathrm{eq}$.

1.2 eq.

1.0 eq.

0.9 eq.

0.8 eq.

0.6 eq.

0.5 eq.

0.3 eq.

0.0 eq.
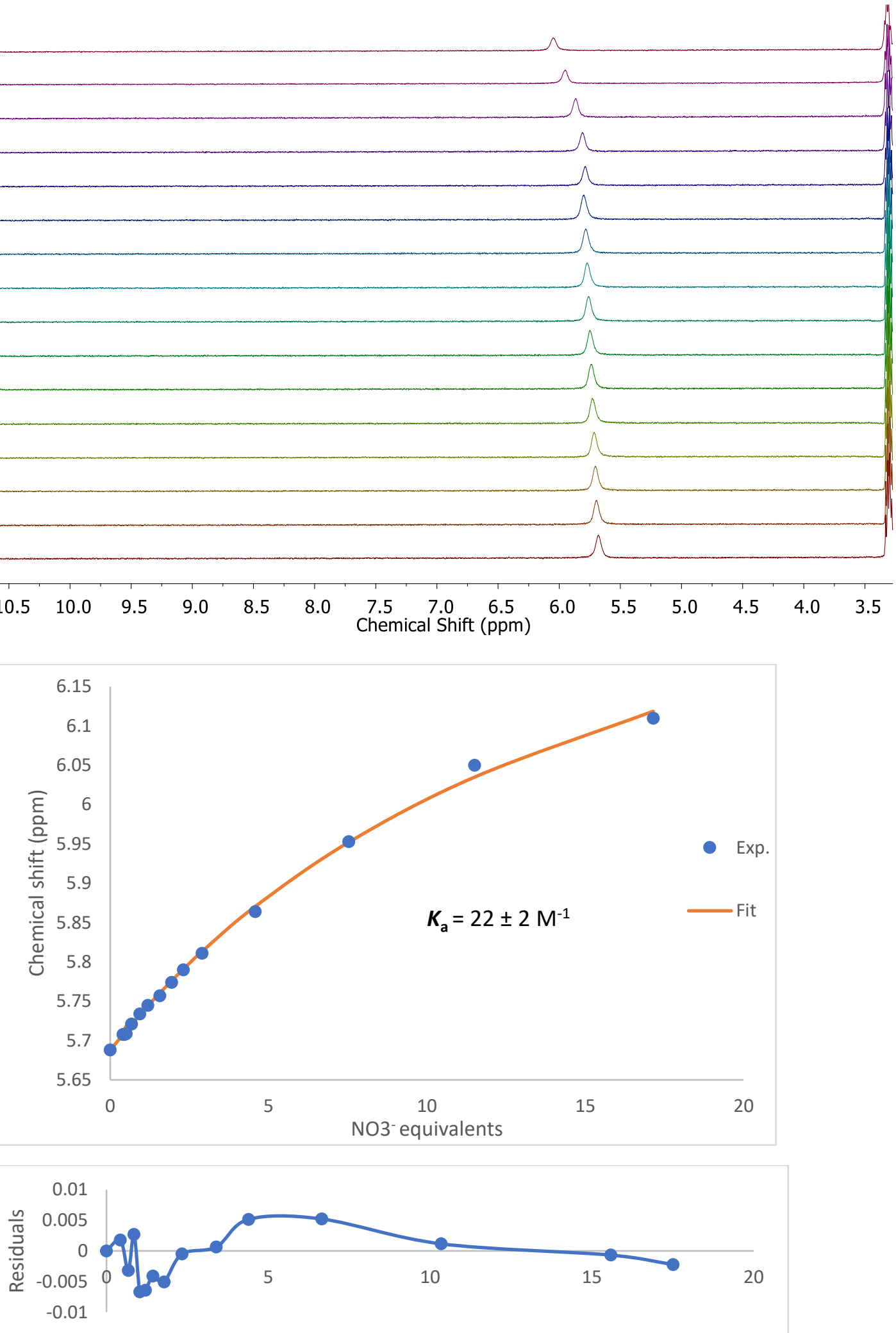

Fig. S21. ${ }^{1} \mathrm{H} \mathrm{NMR}\left(400 \mathrm{MHz}, \mathrm{CD}_{3} \mathrm{CN}\right)$ stack plot of $4(2.7 \mathrm{mM}) \mathrm{TBANO}_{3}$ at $300 \mathrm{~K}, 1: 1$ binding fit, residuals. Average $\mathrm{K}_{\mathrm{a}}$ of two titrations and fitting error shown. 
22.9 eq.

16.8 eq.

11.0 eq.

$6.9 \mathrm{eq}$.

$4.4 \mathrm{eq}$.

$3.2 \mathrm{eq}$.

$2.7 \mathrm{eq}$.

$2.1 \mathrm{eq}$.

$1.6 \mathrm{eq}$.

1.3 eq.

$1.1 \mathrm{eq}$.

$0.9 \mathrm{eq}$.

$0.7 \mathrm{eq}$.

0.5 eq.

0.3 eq.

0.0 eq.
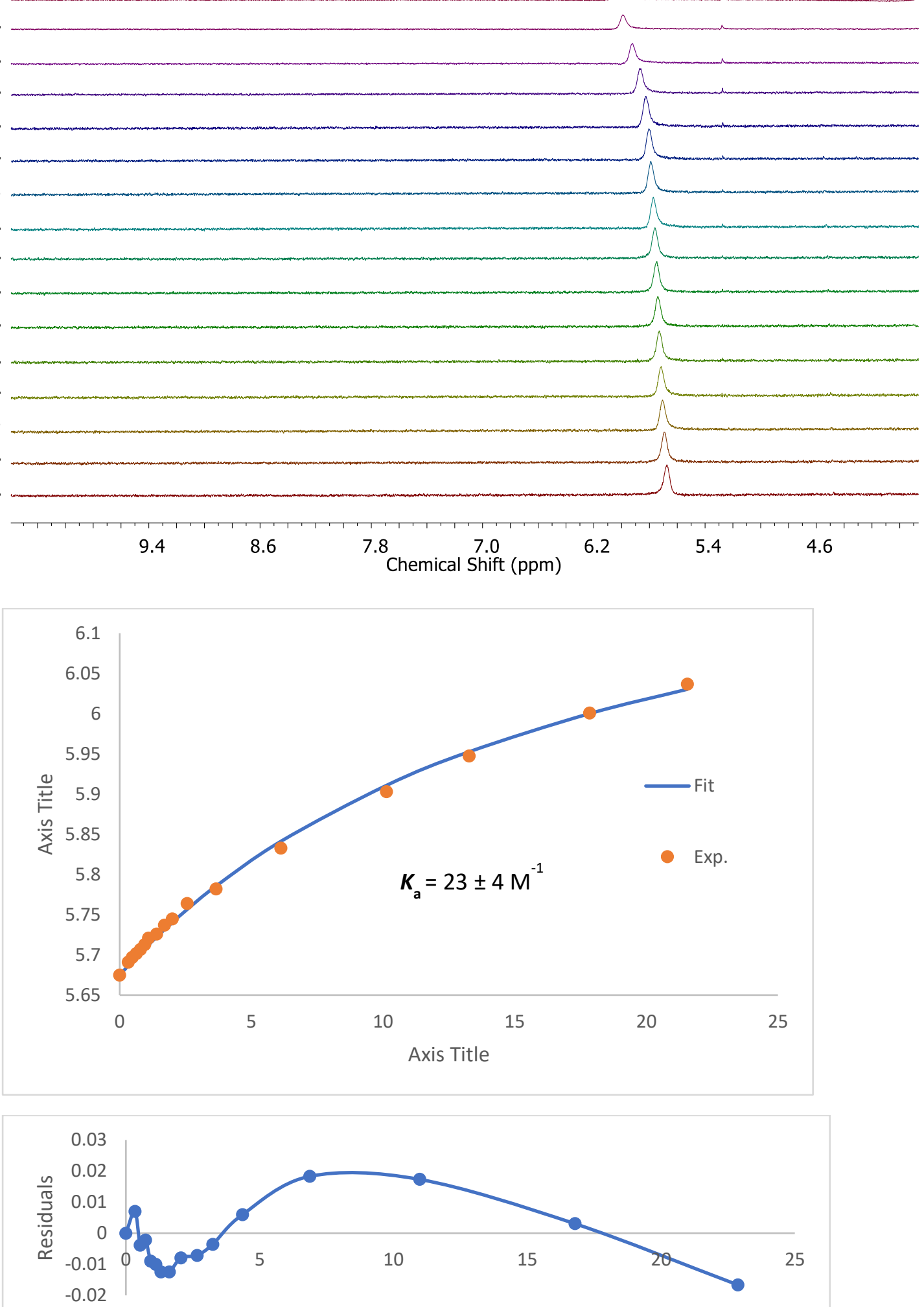

Fig. S22. ${ }^{1} \mathrm{H}$ NMR $\left(400 \mathrm{MHz}, \mathrm{CD}_{3} \mathrm{CN}\right)$ stack plot of $4(2.6 \mathrm{mM}) \mathrm{TBAHSO}_{4}$ at $300 \mathrm{~K}, 1: 1$ binding fit, residuals. Average $\mathrm{K}_{\mathrm{a}}$ of two titrations and fitting error shown. 

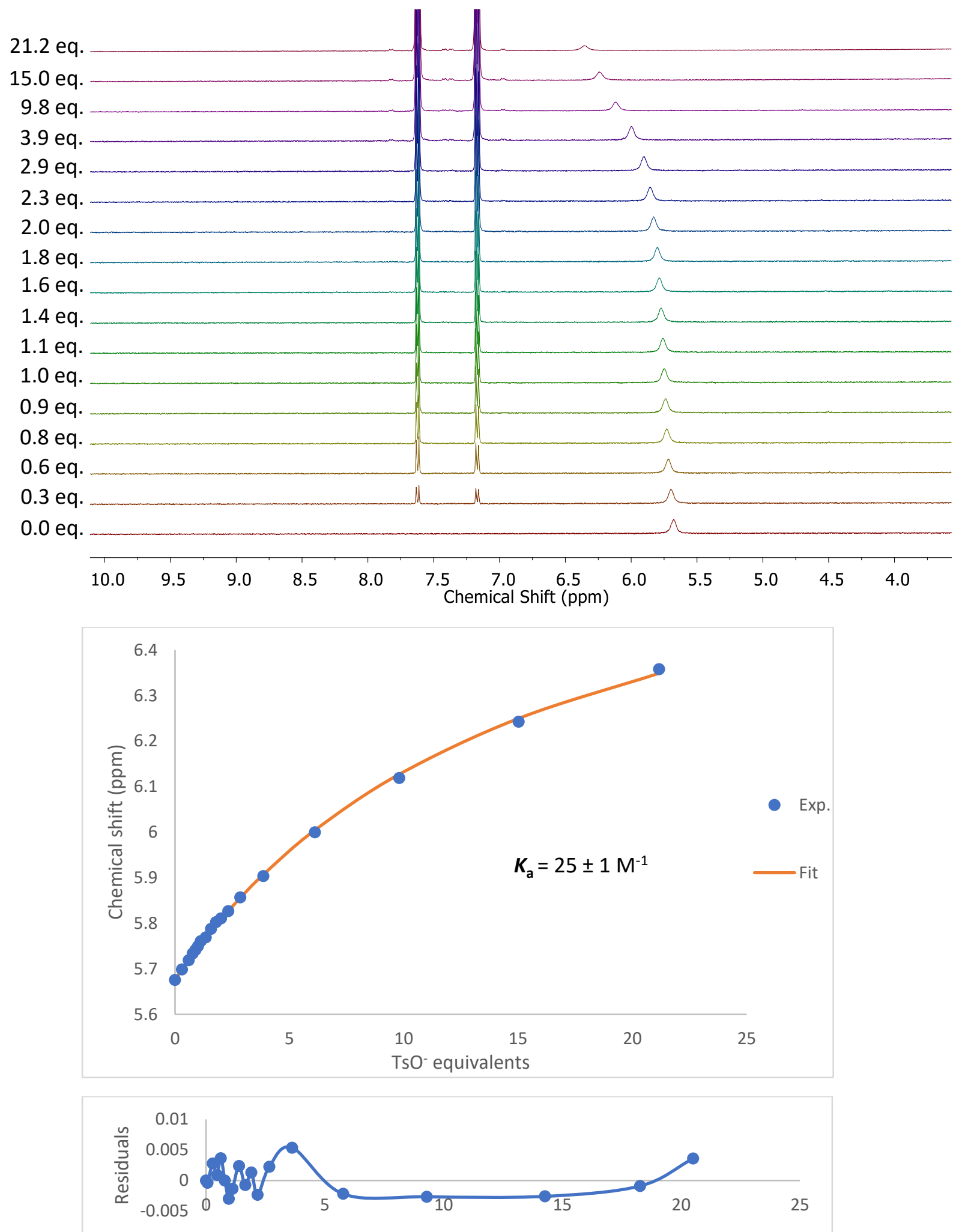

Fig. S23. ${ }^{1} \mathrm{H}$ NMR $\left(400 \mathrm{MHz}, \mathrm{CD}_{3} \mathrm{CN}\right)$ stack plot of $4(2.5 \mathrm{mM})$ TBAOTs at $300 \mathrm{~K}, 1: 1$ binding fit, residuals. Average $K_{a}$ of two titrations and fitting error shown. 

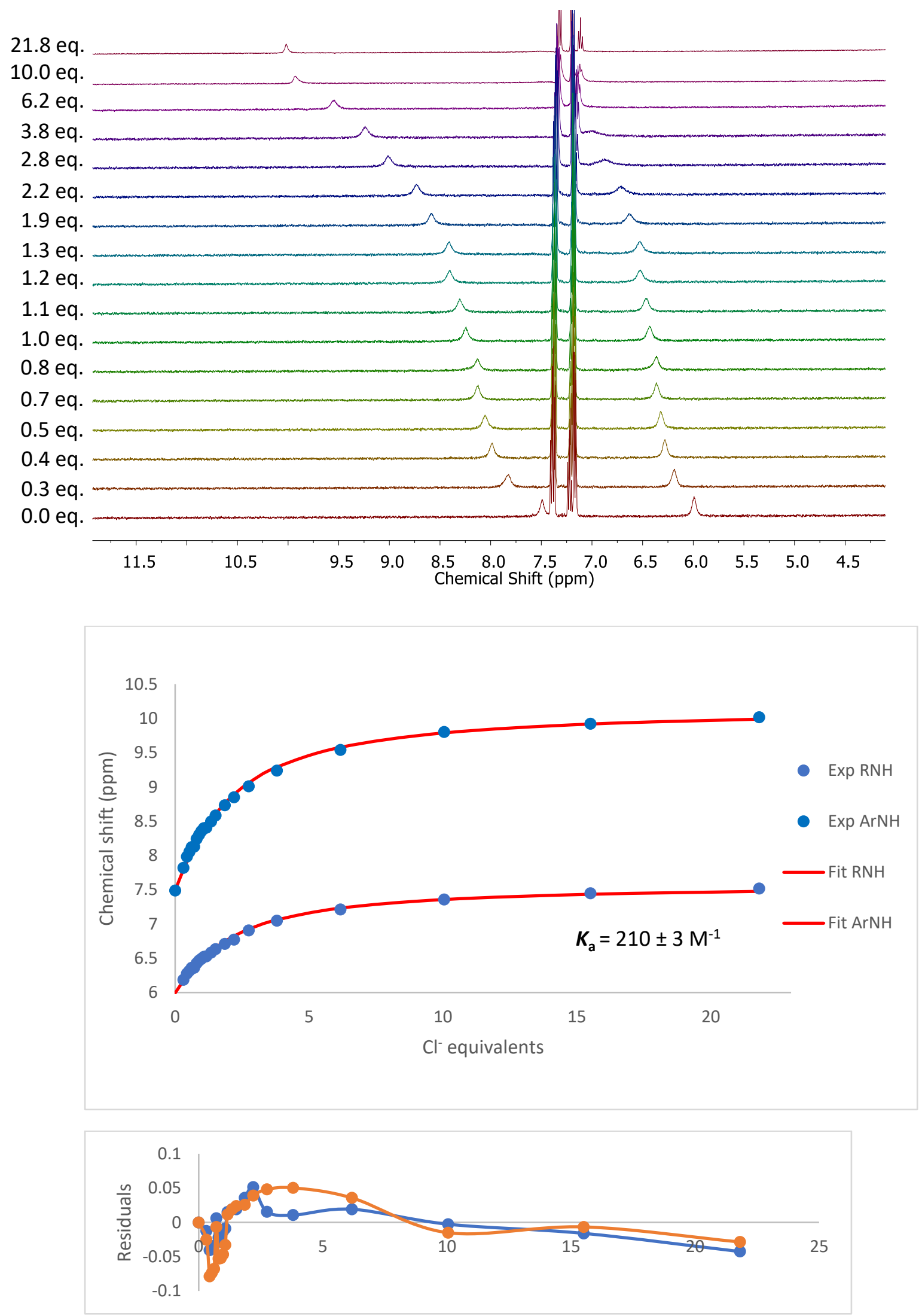

Fig. S24. ${ }^{1} \mathrm{H}$ NMR (400 MHz, $\mathrm{CD}_{3} \mathrm{CN}$ ) stack plot of 5 (3.1 mM) TBACl at $300 \mathrm{~K}, 1: 1$ binding, residuals. Average $\mathrm{K}_{\mathrm{a}}$ of two titrations and fitting error shown. 

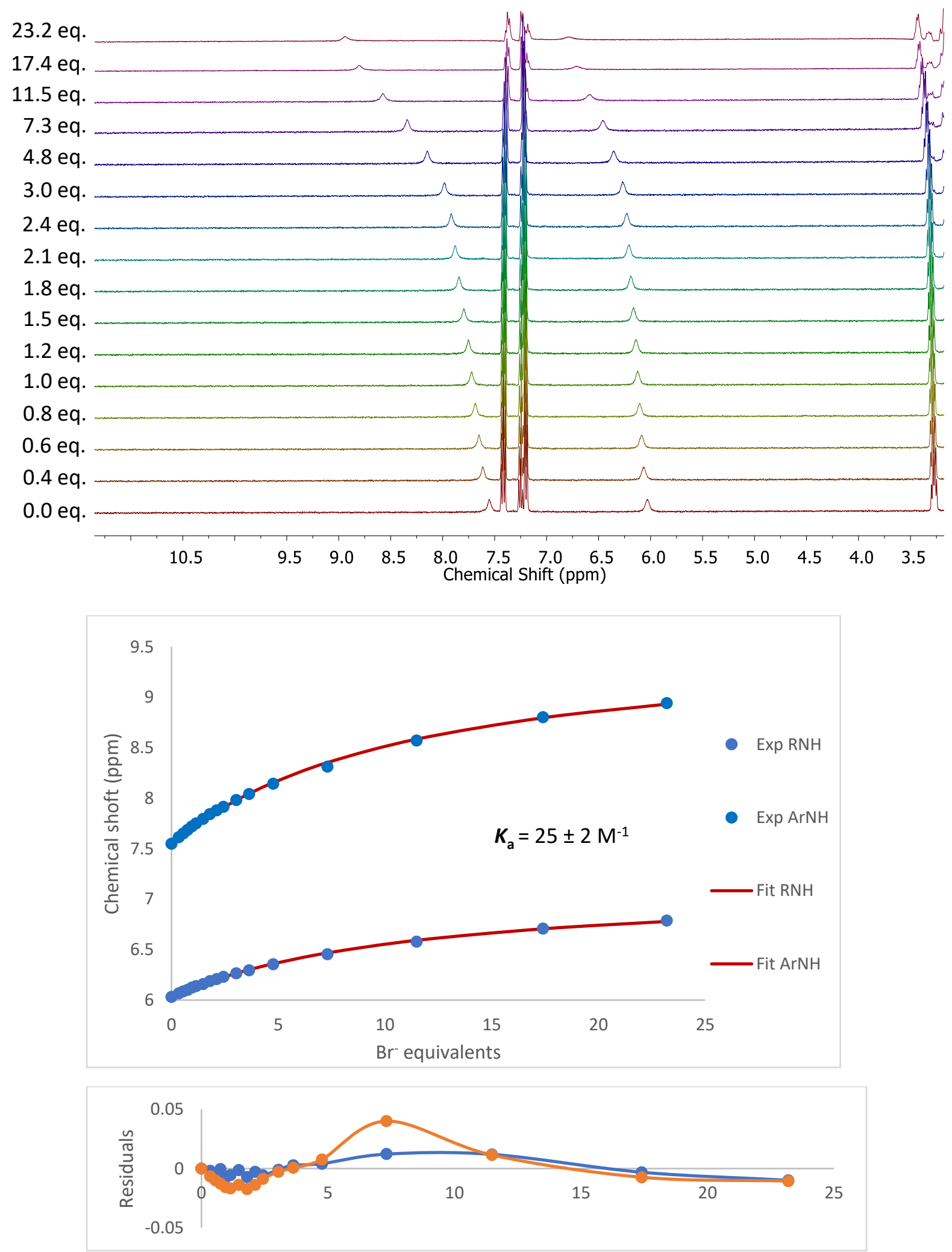

Fig. S25. ${ }^{1} \mathrm{H}$ NMR ( $400 \mathrm{MHz}, \mathrm{CD}_{3} \mathrm{CN}$ ) stack plot of 5 ( $\left.2.6 \mathrm{mM}\right) \mathrm{TBABr}$ at $300 \mathrm{~K}, 1: 1$ binding fit, residuals. Average $\mathrm{K}_{\mathrm{a}}$ of two titrations and fitting error shown. 

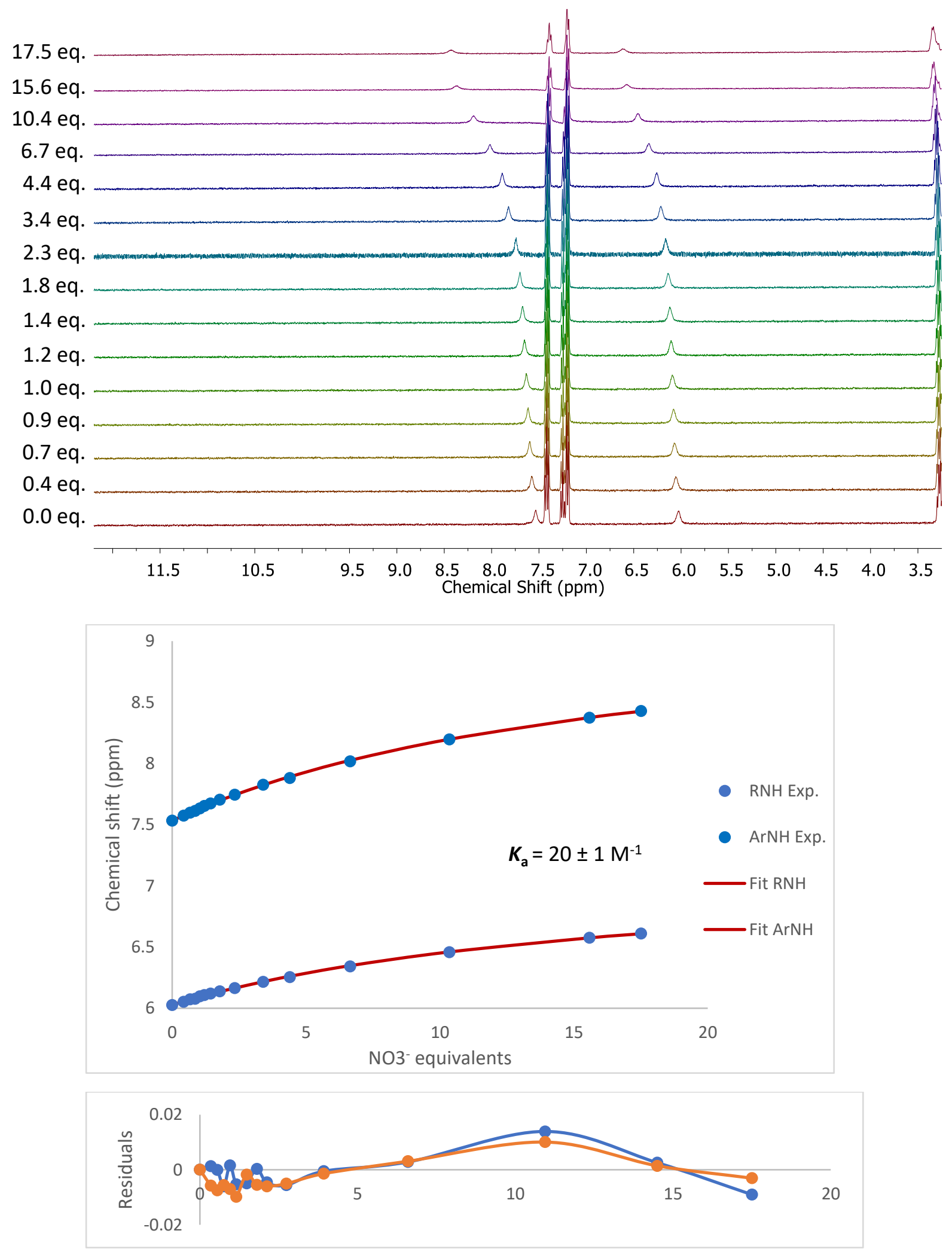

Fig. S26. ${ }^{1} \mathrm{H}$ NMR $\left(400 \mathrm{MHz}, \mathrm{CD}_{3} \mathrm{CN}\right)$ stack plot of $5(2.8 \mathrm{mM}) \mathrm{TBANO}_{3}$ at $300 \mathrm{~K}, 1: 1$ binding fit, residuals. Average $\mathrm{K}_{\mathrm{a}}$ of two titrations and fitting error shown. 

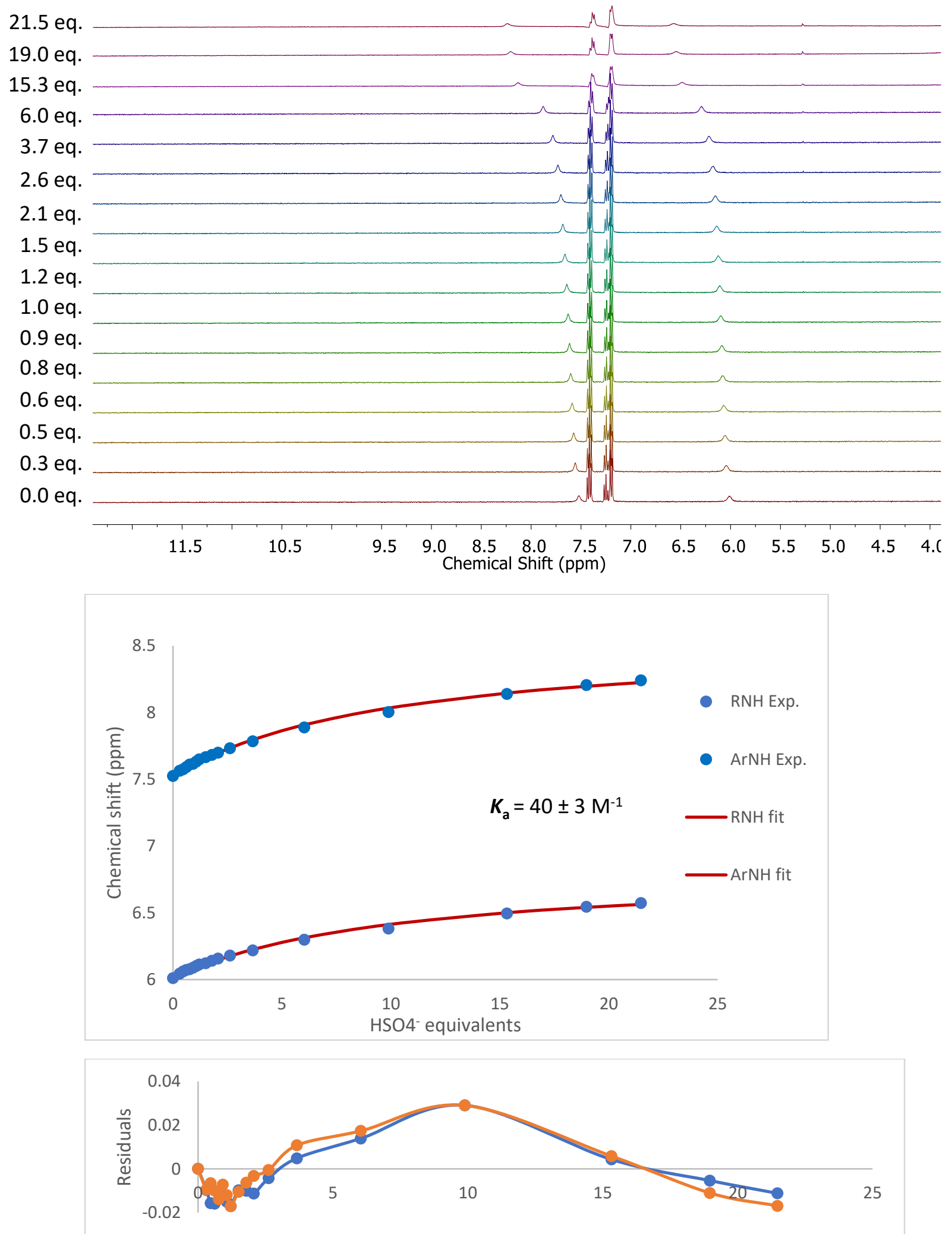

Fig. S27. ${ }^{1} \mathrm{H}$ NMR $\left(400 \mathrm{MHz}, \mathrm{CD}_{3} \mathrm{CN}\right)$ stack plot of $5(2.7 \mathrm{mM}) \mathrm{TBAHSO}_{4}$ at $300 \mathrm{~K}, 1: 1$ binding fit, residuals. Average $K_{a}$ of two titrations and fitting error shown. 

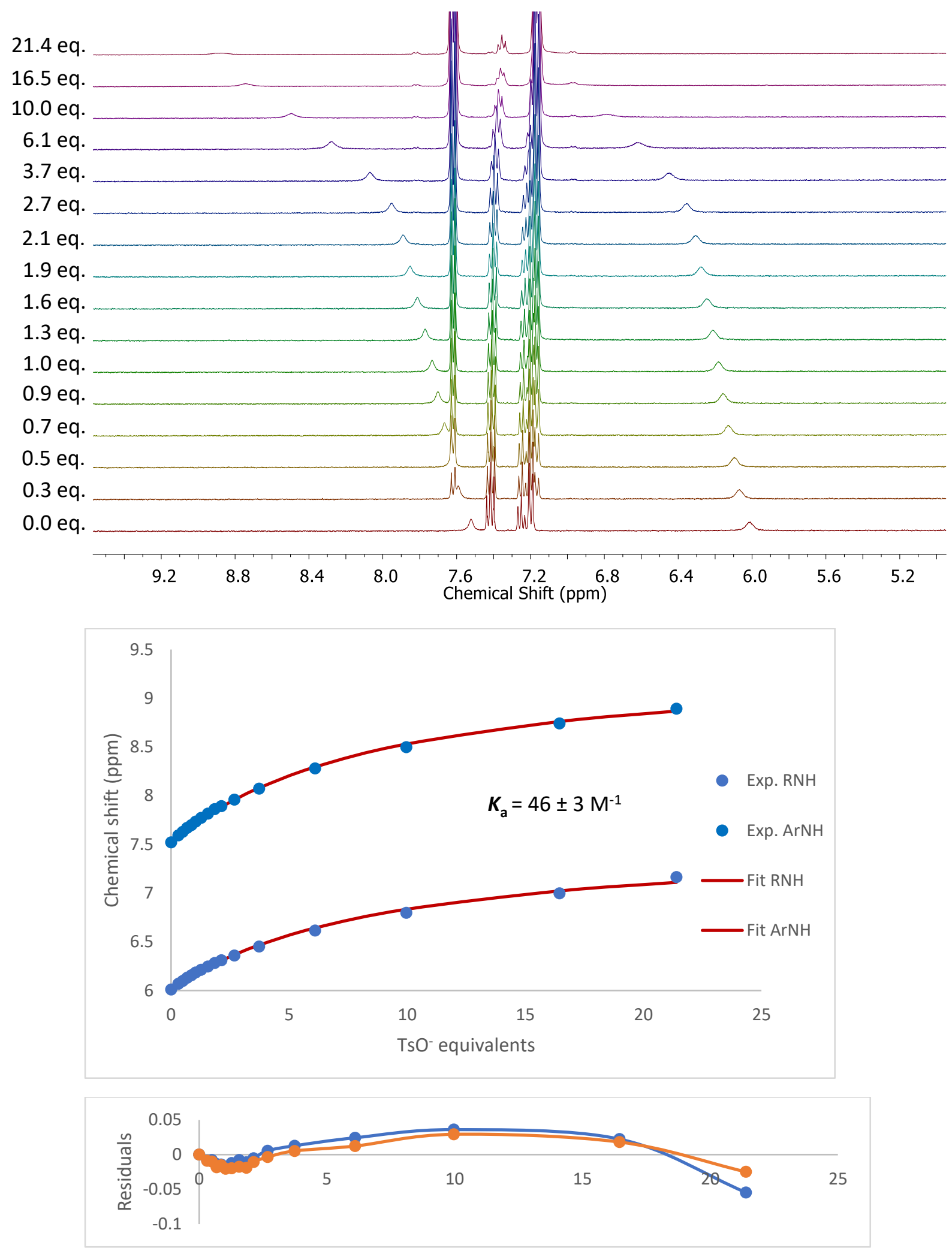

Fig. S28. ${ }^{1} \mathrm{H}$ NMR ( $\left.400 \mathrm{MHz}, \mathrm{CD}_{3} \mathrm{CN}\right)$ stack plot of $5(2.7 \mathrm{mM})$ TBAOTs at $300 \mathrm{~K}, 1: 1$ binding fit, residuals. Average $\mathrm{K}_{\mathrm{a}}$ of two titrations and fitting error shown. 

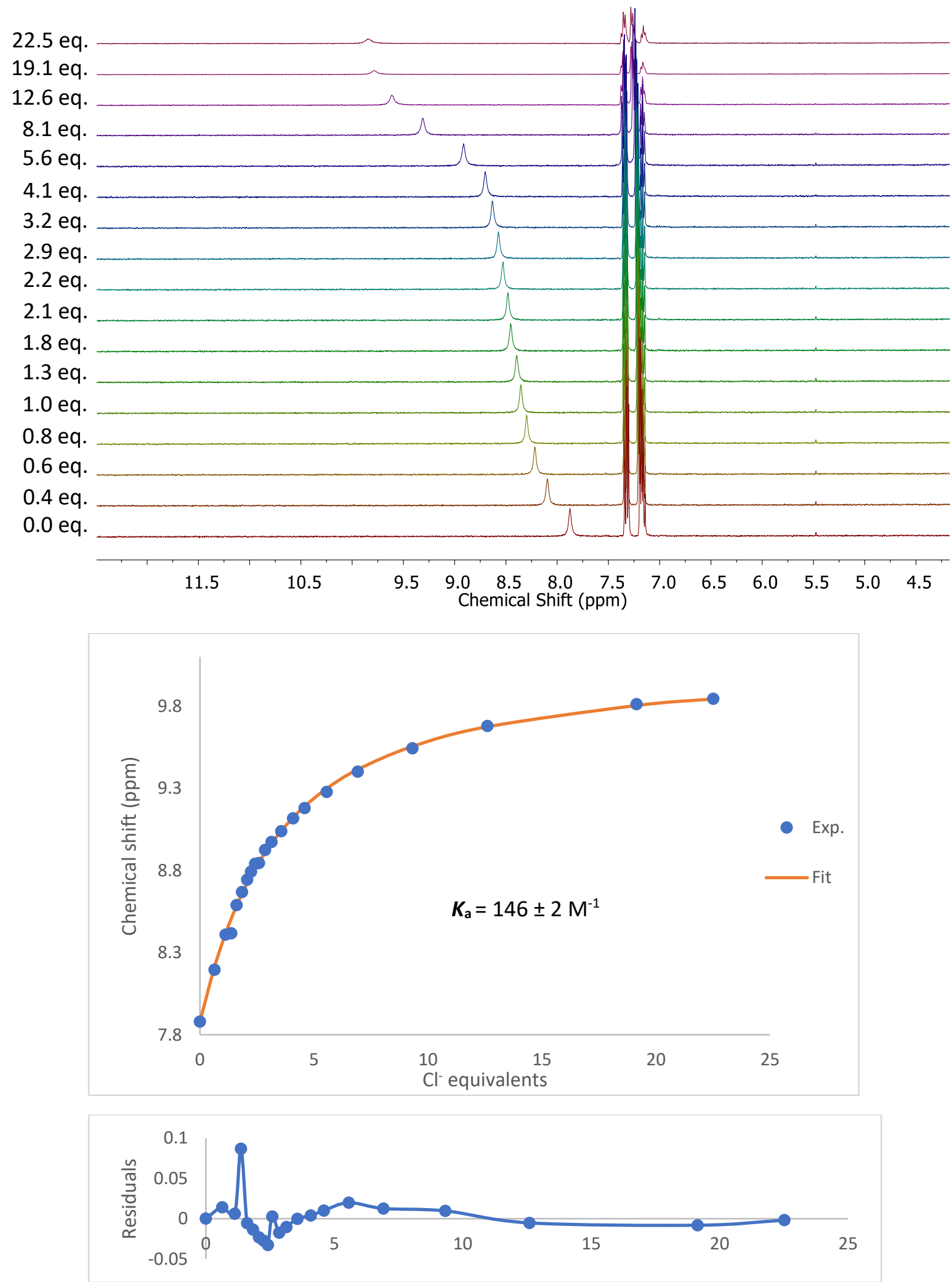

Fig. S29. ${ }^{1} \mathrm{H}$ NMR (400 MHz, $\left.\mathrm{CD}_{3} \mathrm{CN}\right)$ stack plot of 6 ( $\left.3.8 \mathrm{mM}\right)$ TBACl at $300 \mathrm{~K}, 1: 1$ binding fit, residuals. Average $\mathrm{K}_{\mathrm{a}}$ of two titrations and fitting error shown. 

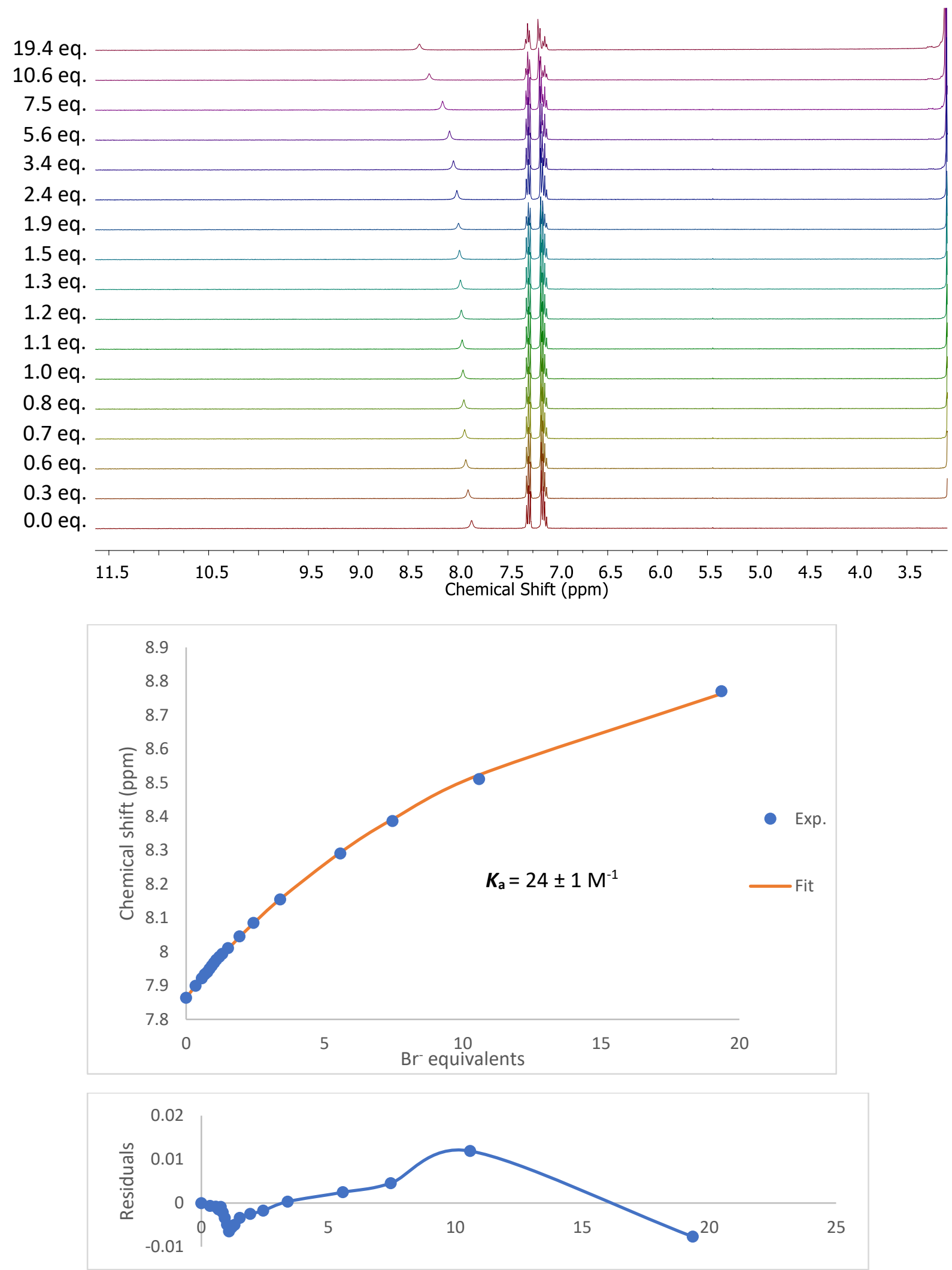

Fig. S30. ${ }^{1} \mathrm{H}$ NMR $\left(400 \mathrm{MHz}, \mathrm{CD}_{3} \mathrm{CN}\right)$ stack plot of $6(3.0 \mathrm{mM}) \mathrm{TBABr}$ at $300 \mathrm{~K}, 1: 1$ binding fit, residuals. Average $\mathrm{K}_{\mathrm{a}}$ of two titrations and fitting error shown. 

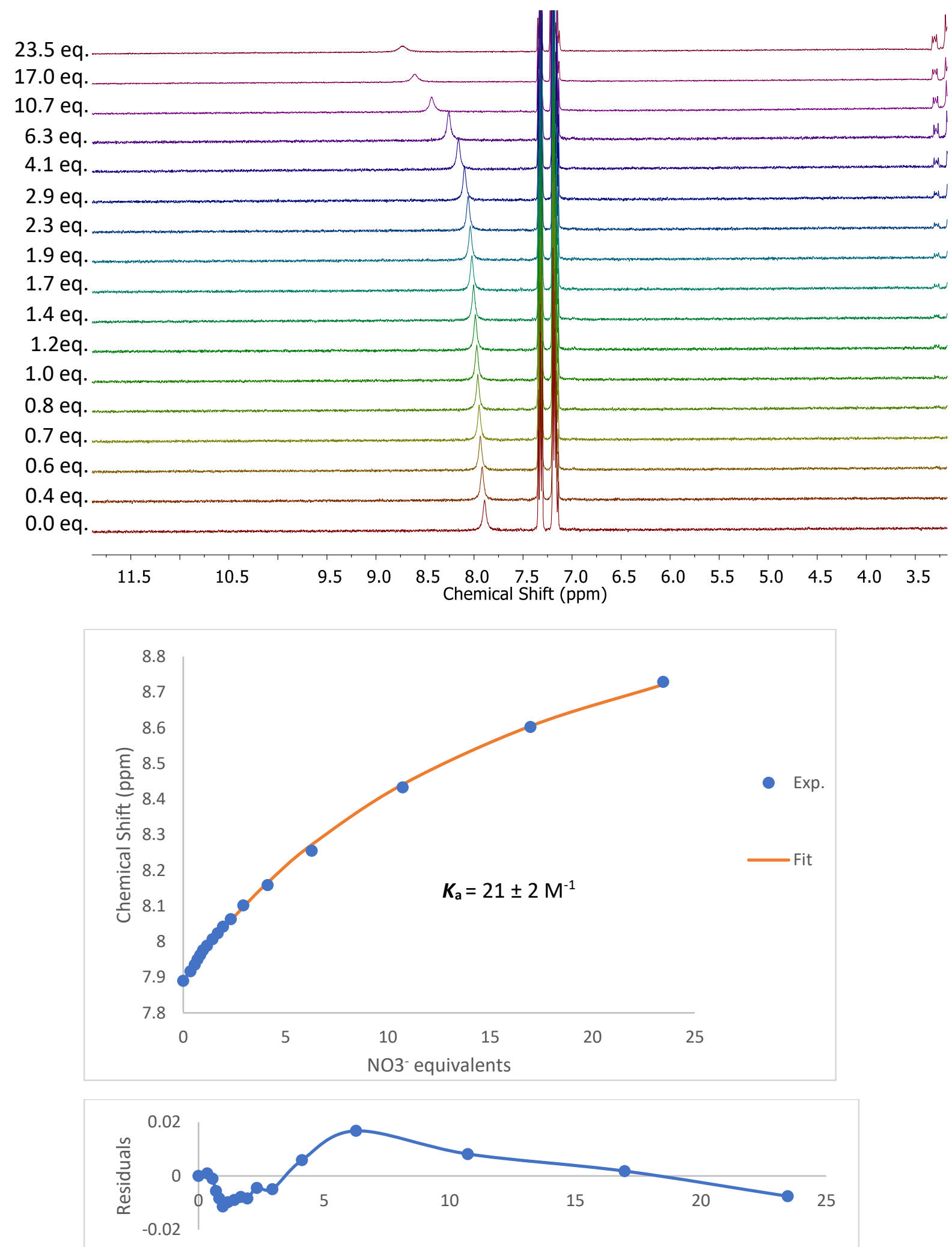

Fig. S31. ${ }^{1} \mathrm{H}$ NMR $\left(400 \mathrm{MHz}, \mathrm{CD}_{3} \mathrm{CN}\right)$ stack plot of $6(2.8 \mathrm{mM}) \mathrm{TBANO}_{3}$ at $300 \mathrm{~K}, 1: 1$ binding fit, residuals. Average $\mathrm{K}_{\mathrm{a}}$ of two titrations and fitting error shown. 

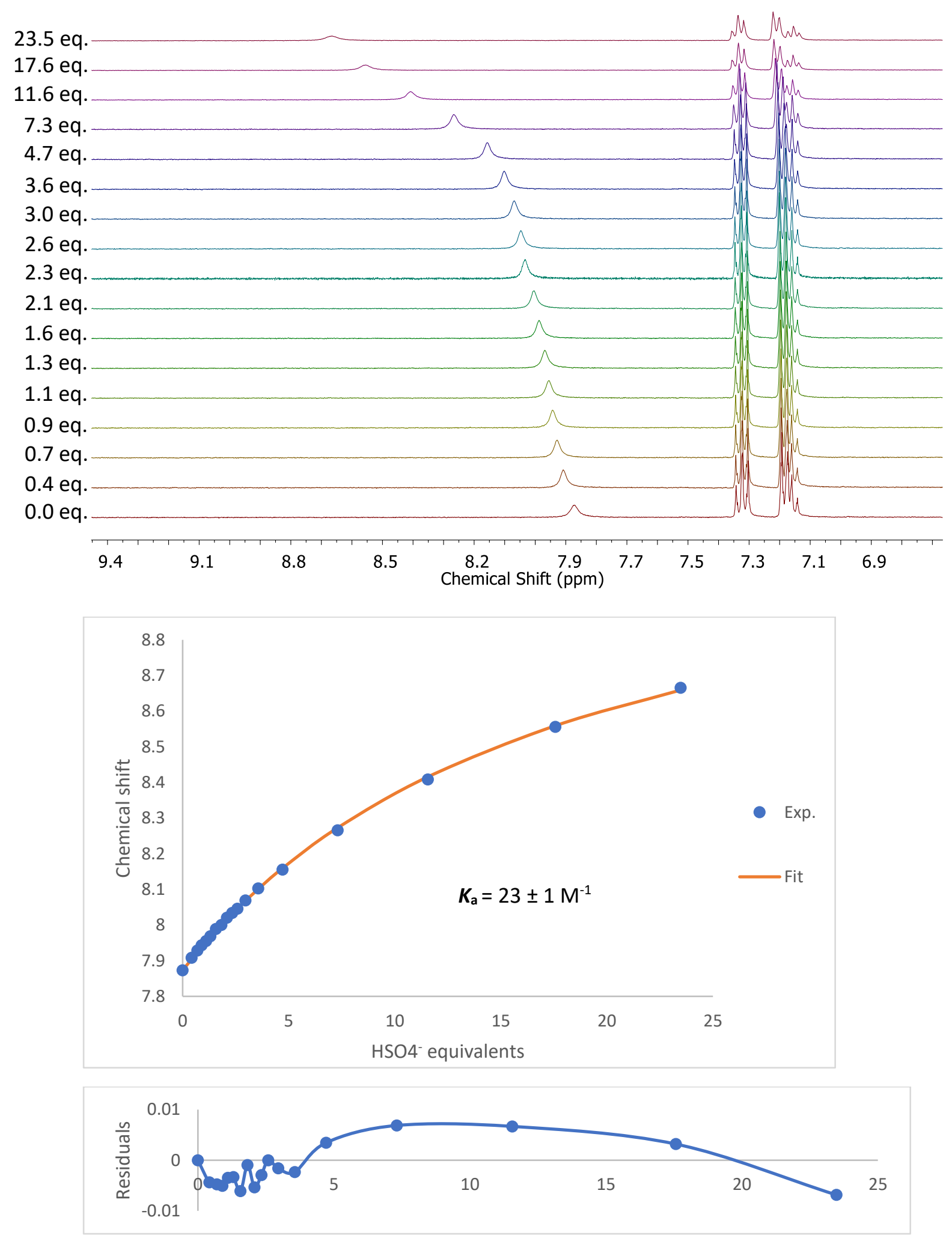

Fig. S32. ${ }^{1} \mathrm{H}$ NMR (400 MHz, $\left.\mathrm{CD}_{3} \mathrm{CN}\right)$ stack plot of $6(2.6 \mathrm{mM}) \mathrm{TBAHSO}_{4}$ at $300 \mathrm{~K}, 1: 1$ binding fit, residuals. Average $K_{a}$ of two titrations and fitting error shown. 

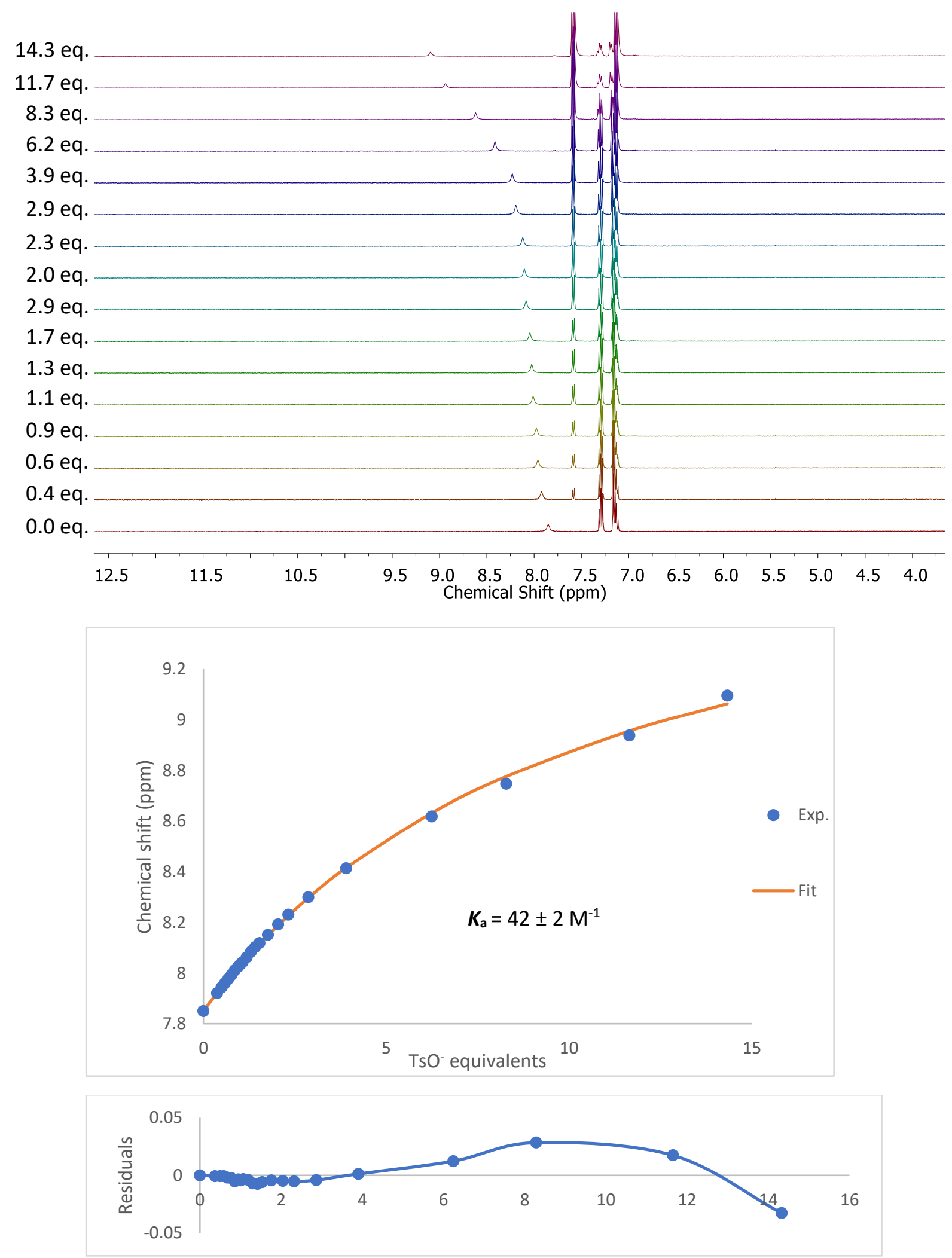

Fig. S33. ${ }^{1} \mathrm{H}$ NMR (400 MHz, $\mathrm{CD}_{3} \mathrm{CN}$ ) stack plot of 6 (3.7 mM) TBAOTs at $300 \mathrm{~K}, 1: 1$ binding fit, residuals. Average $K_{a}$ of two titrations and fitting error shown. 


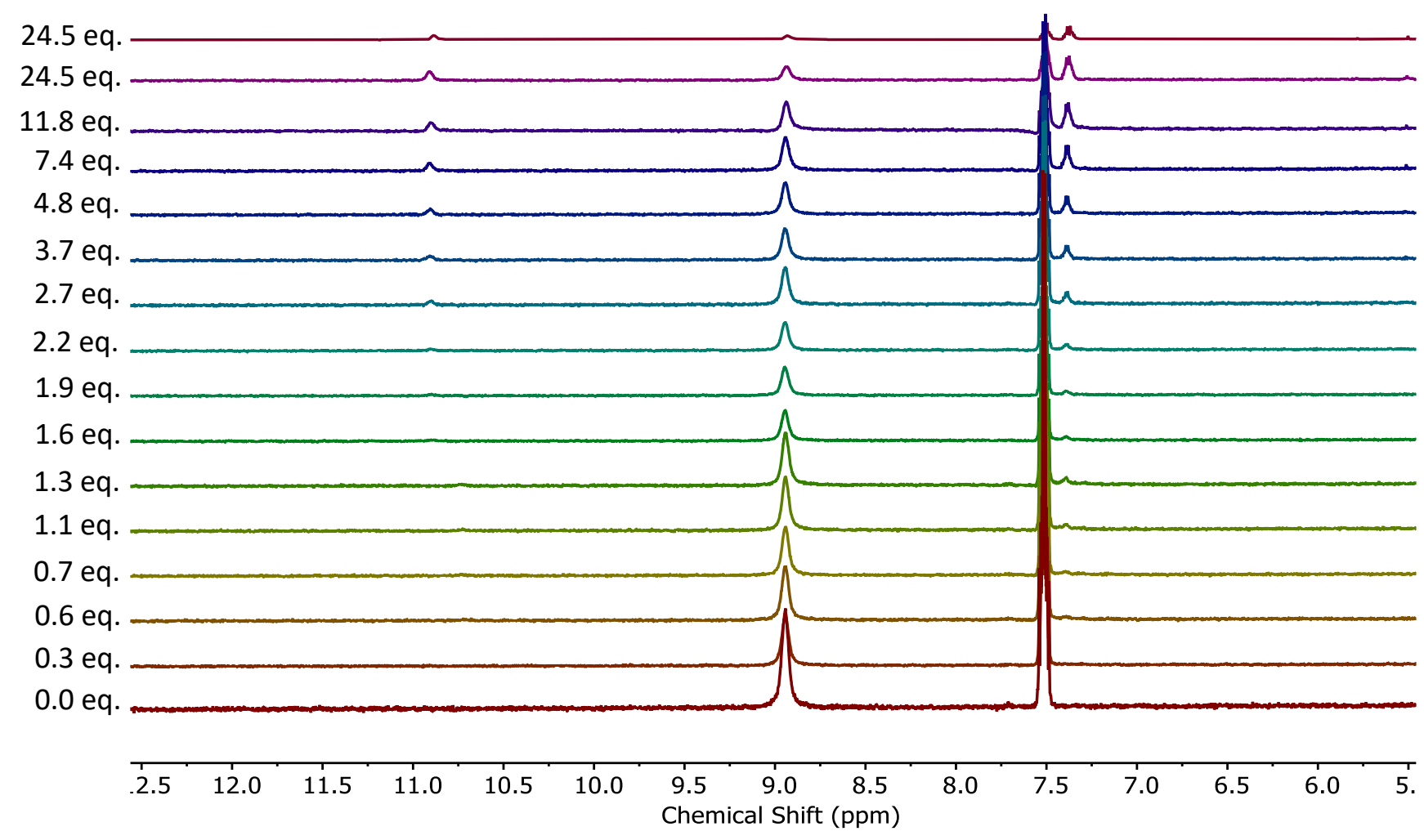

$\mathrm{K}_{\mathrm{a}}=\frac{[H G]}{[H][G]}$

Slow exchange binding. $\mathrm{K}_{\mathrm{a}}$ calculated directly from relative integration ratios.

$$
K_{\mathrm{a}}=10 \mathrm{M}^{-1}
$$

Fig. S34. ${ }^{1} \mathrm{H}$ NMR (400 MHz, CD ${ }_{3} \mathrm{CN}$ ) stack plot of 7 (3.3 mM) $\mathrm{TBA}_{2} \mathrm{SO}_{4}$ at $300 \mathrm{~K}, \mathrm{~K}_{\mathrm{a}}$ calculated. Average $\mathrm{K}_{\mathrm{a}}$ of two titrations shown. 


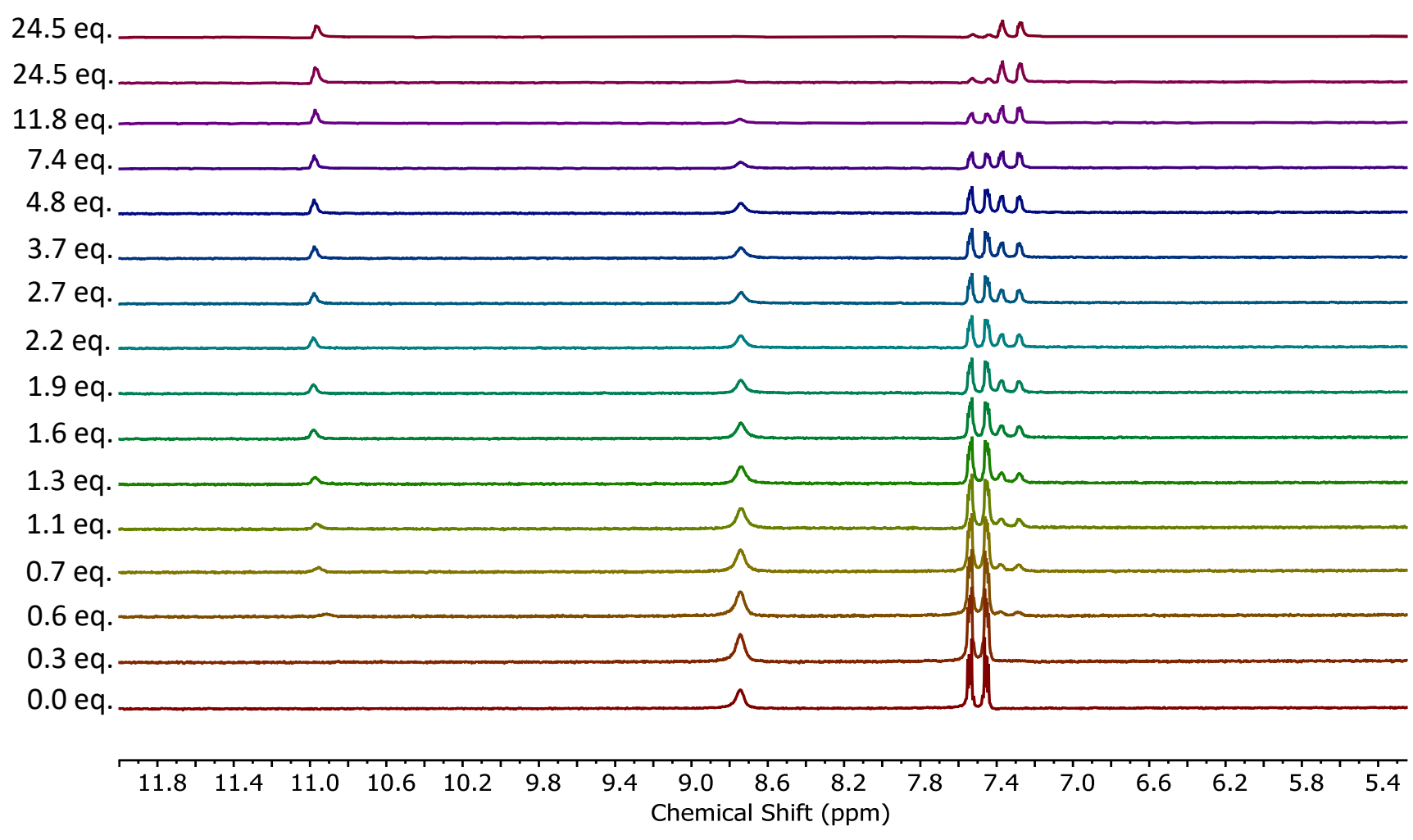

$\mathrm{K}_{\mathrm{a}}=\frac{[H G]}{[H][G]}$

$\mathrm{K}_{\mathrm{a}}$ calculated directly from relative integration ratios.

$K_{\mathrm{a}}=180 \mathrm{M}^{-1}$

Fig. S35. ${ }^{1} \mathrm{H}$ NMR (400 MHz, DMSO-d 6 ) stack plot of $7(2.4 \mathrm{mM}) \mathrm{TBA}_{2} \mathrm{SO}_{4}$ at $300 \mathrm{~K}, \mathrm{~K}_{\mathrm{a}}$ calculated. Average $\mathrm{K}_{\mathrm{a}}$ of two titrations shown. 

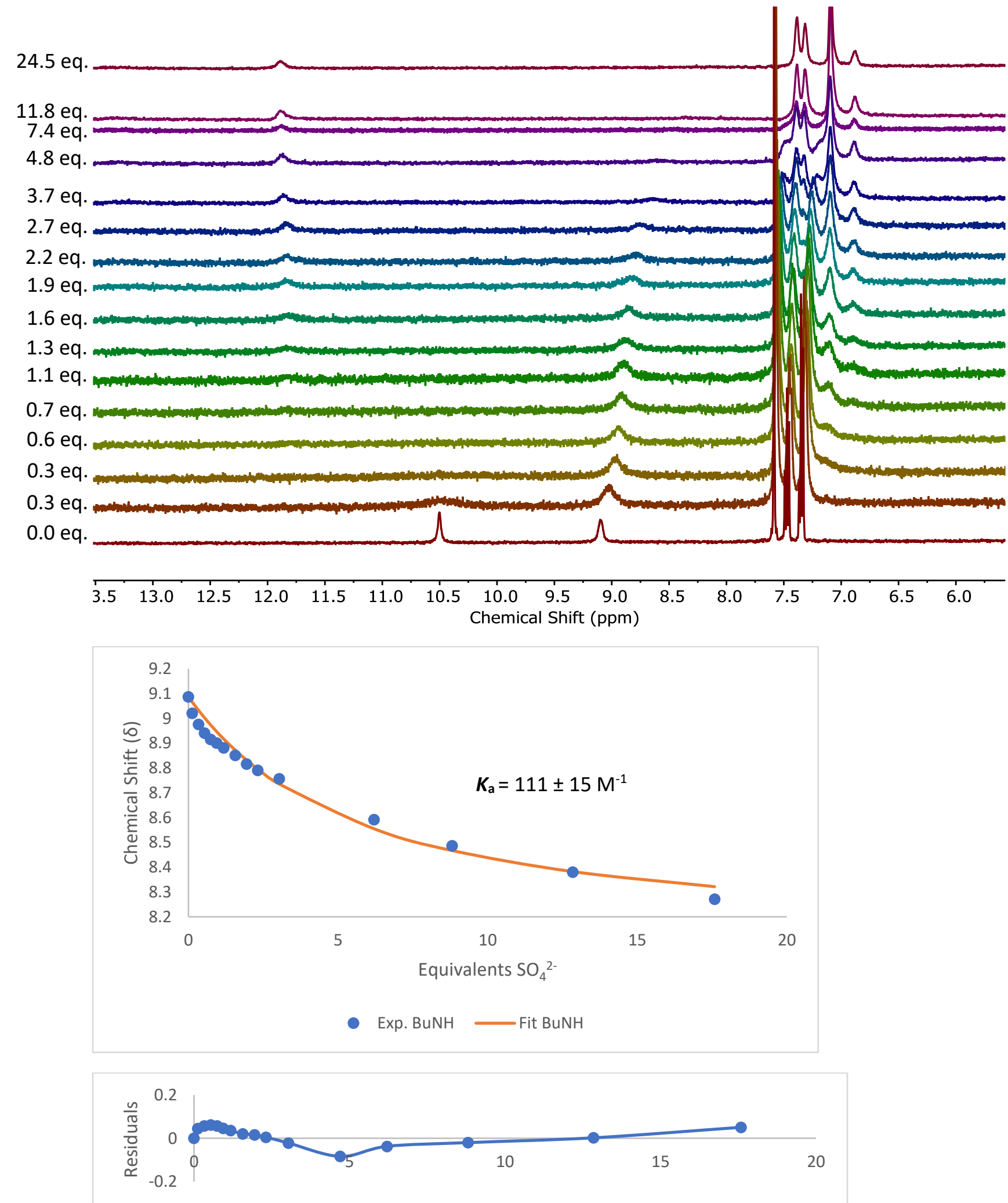

Fig. S36. ${ }^{1} \mathrm{H}$ NMR (400 MHz, $\mathrm{CD}_{3} \mathrm{CN}$ ) stack plot of $8\left(2.3 \mathrm{mM}\right.$ ) $\mathrm{TBA}_{2} \mathrm{SO}_{4}$ at $300 \mathrm{~K}, 1: 1$ binding fit, residuals. Average $\mathrm{K}_{\mathrm{a}}$ of two titrations and fitting error shown. 
24.5 eq

$11.8 \mathrm{eq}$

$7.4 \mathrm{eq}$

$4.8 \mathrm{eq}$

$3.7 \mathrm{eq}$

$2.7 \mathrm{eq}$

$2.2 \mathrm{eq}$

1.9 eq

$1.6 \mathrm{eq}$

1.3 eq.

$1.3 \mathrm{eq}$

$1.1 \mathrm{eq}$

0.7 eq.

$0.6 \mathrm{eq}$

0.3 eq.
0.0 eq.
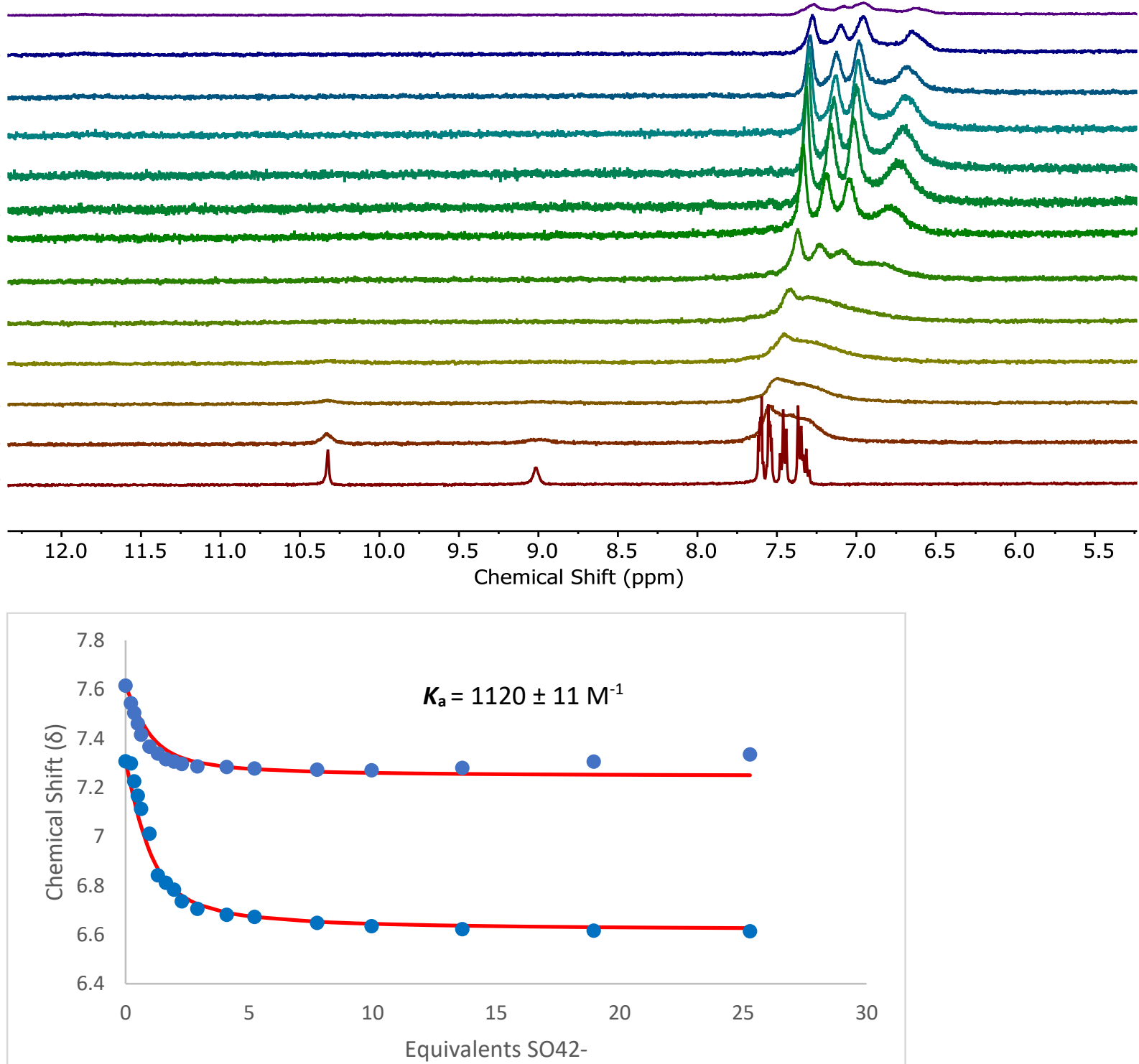

- Exp. $\mathrm{ArH} 1$

- Exp. $\mathrm{ArH} 2 \longrightarrow$ Fit $\mathrm{ArH} 1$

$\longrightarrow$ Fit ArH2

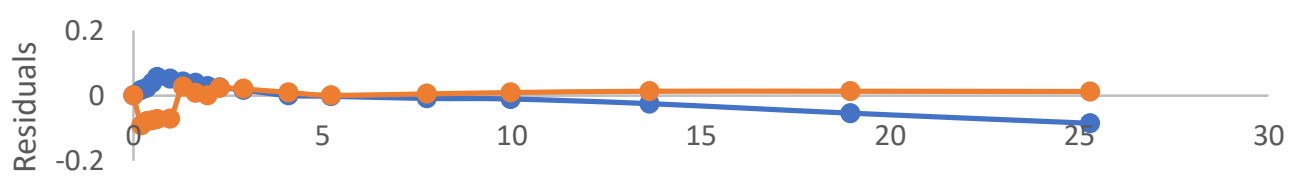

Fig. S37. ${ }^{1} \mathrm{H}$ NMR $\left(400 \mathrm{MHz}\right.$, DMSO-d 6 ) stack plot of $8(2.3 \mathrm{mM}) \mathrm{TBA}_{2} \mathrm{SO}_{4}$ at $300 \mathrm{~K}, 1: 1$ binding fit, residuals. Average $\mathrm{K}_{\mathrm{a}}$ of two titrations and fitting error shown. 
24.5 eq.
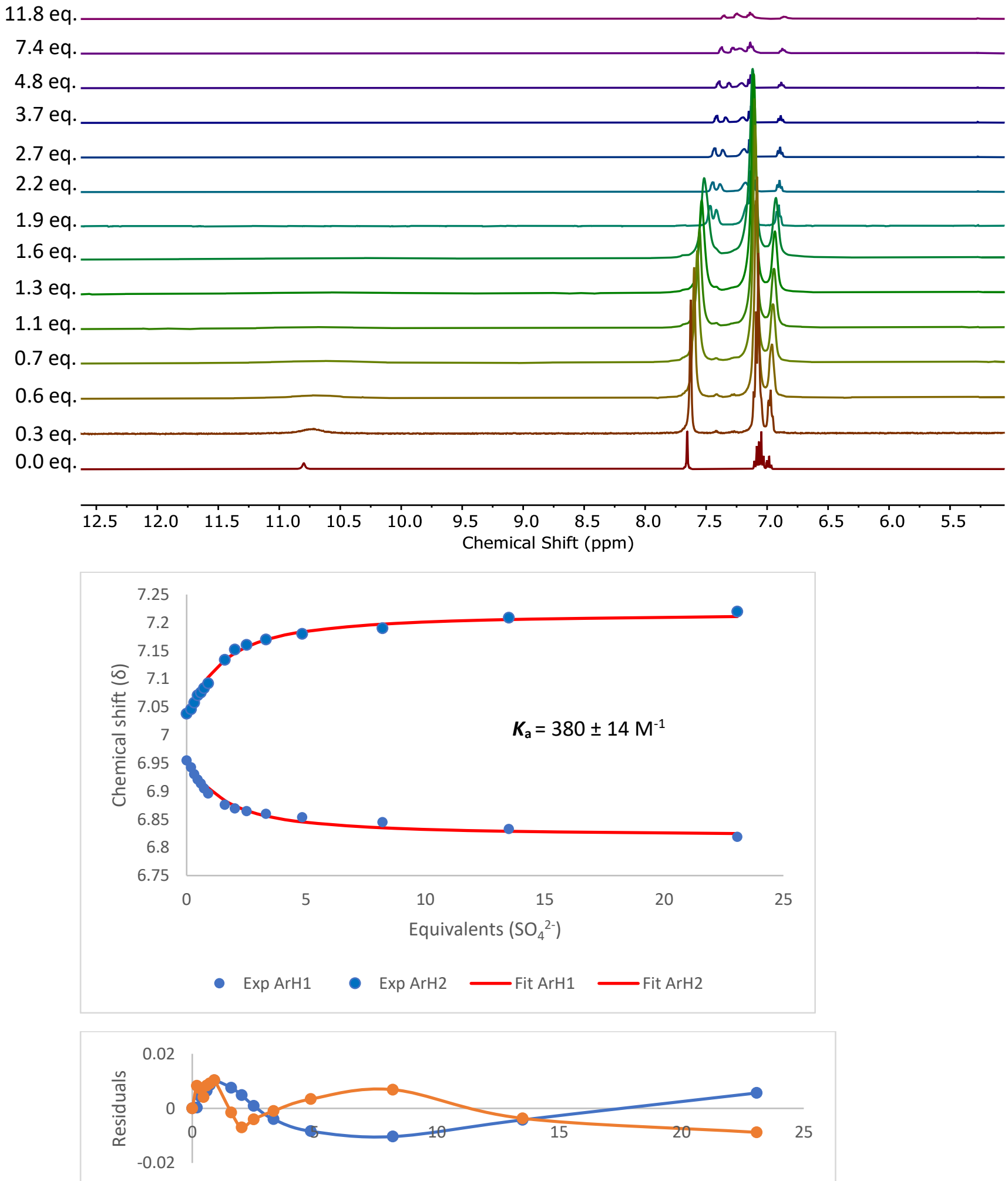

Fig. S38. ${ }^{1} \mathrm{H} N M R\left(400 \mathrm{MHz}, \mathrm{CD}_{3} \mathrm{CN}\right)$ stack plot of $9(2.3 \mathrm{mM}) \mathrm{TBA}_{2} \mathrm{SO}_{4}$ at $300 \mathrm{~K}, 1: 1$ binding, residuals. 


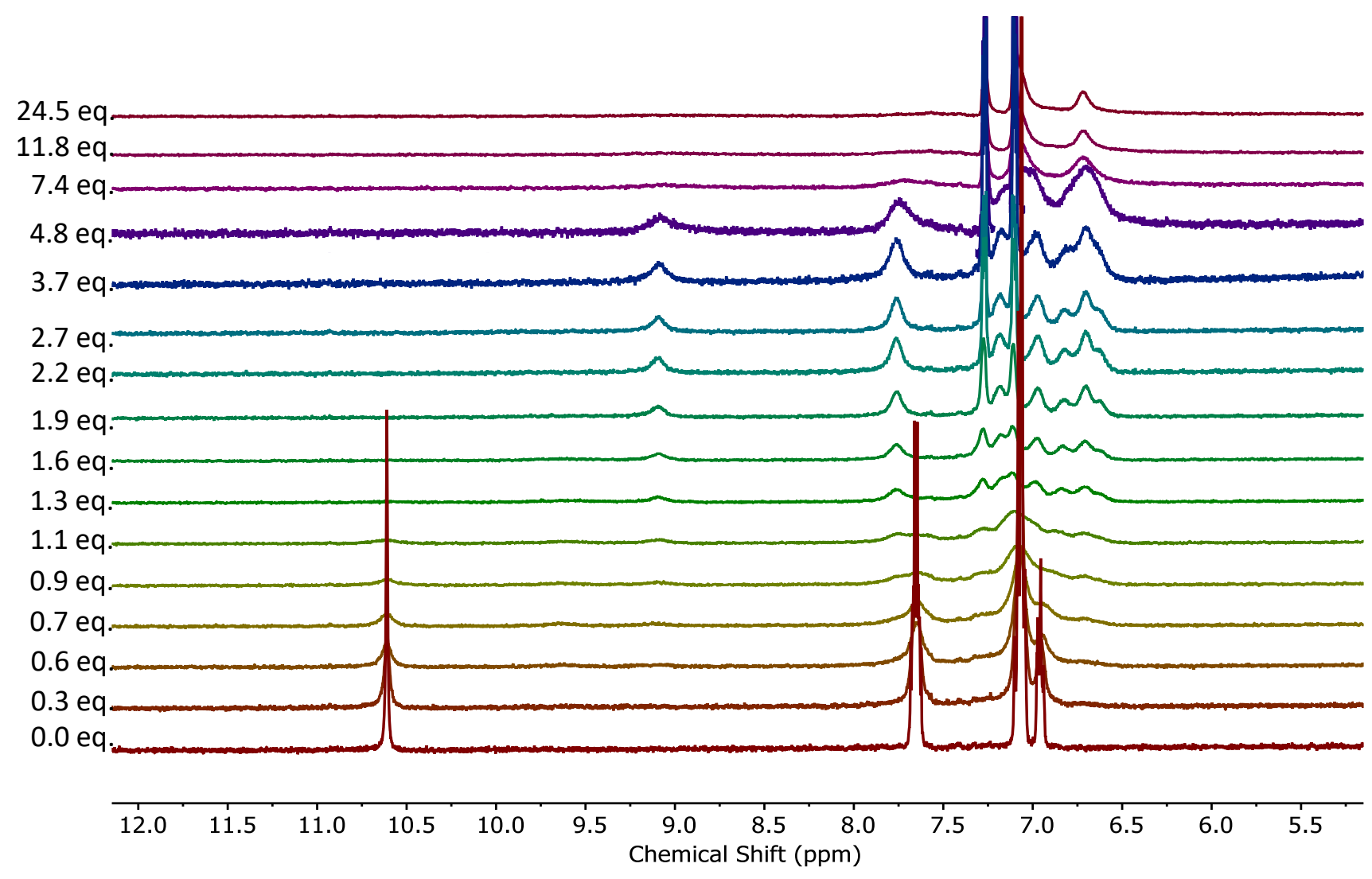

Probable deprotonation due to similarities with $\mathrm{F}^{-}$titration.

Fig. S39. ${ }^{1} \mathrm{H}$ NMR (400 MHz, DMSO-d $)$ stack plot of $9(2.3 \mathrm{mM}) \mathrm{TBA}_{2} \mathrm{SO}_{4}$ at $300 \mathrm{~K}$. 


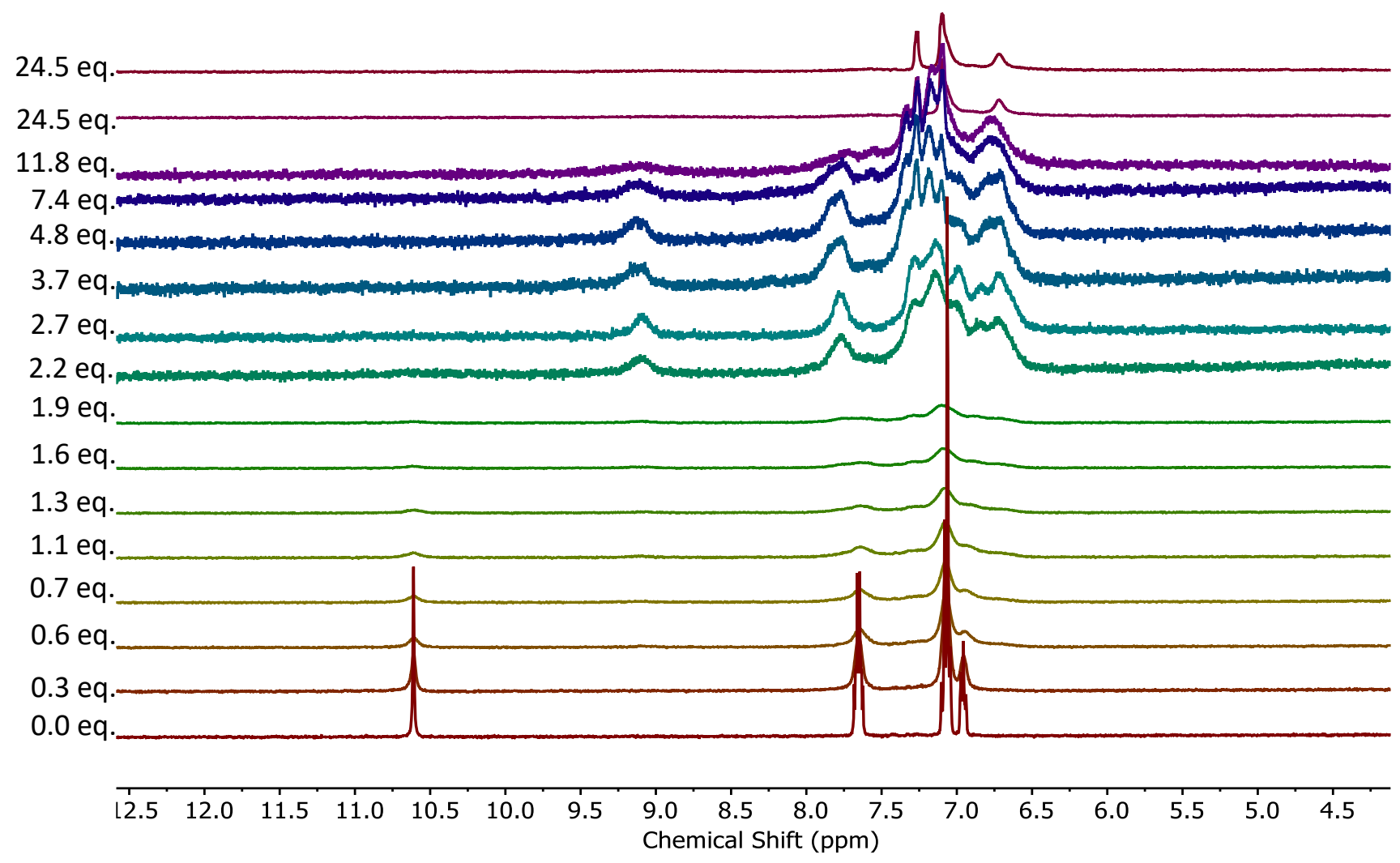

Receptor deprotonates.

Fig. S40. ${ }^{1} \mathrm{H}$ NMR (400 MHz, DMSO- $\left.\mathrm{d}_{6}\right)$ stack plot of $9(2.3 \mathrm{mM})$ TBAF at $300 \mathrm{~K}$. 
High resolution mass spectrometry (HRMS) data

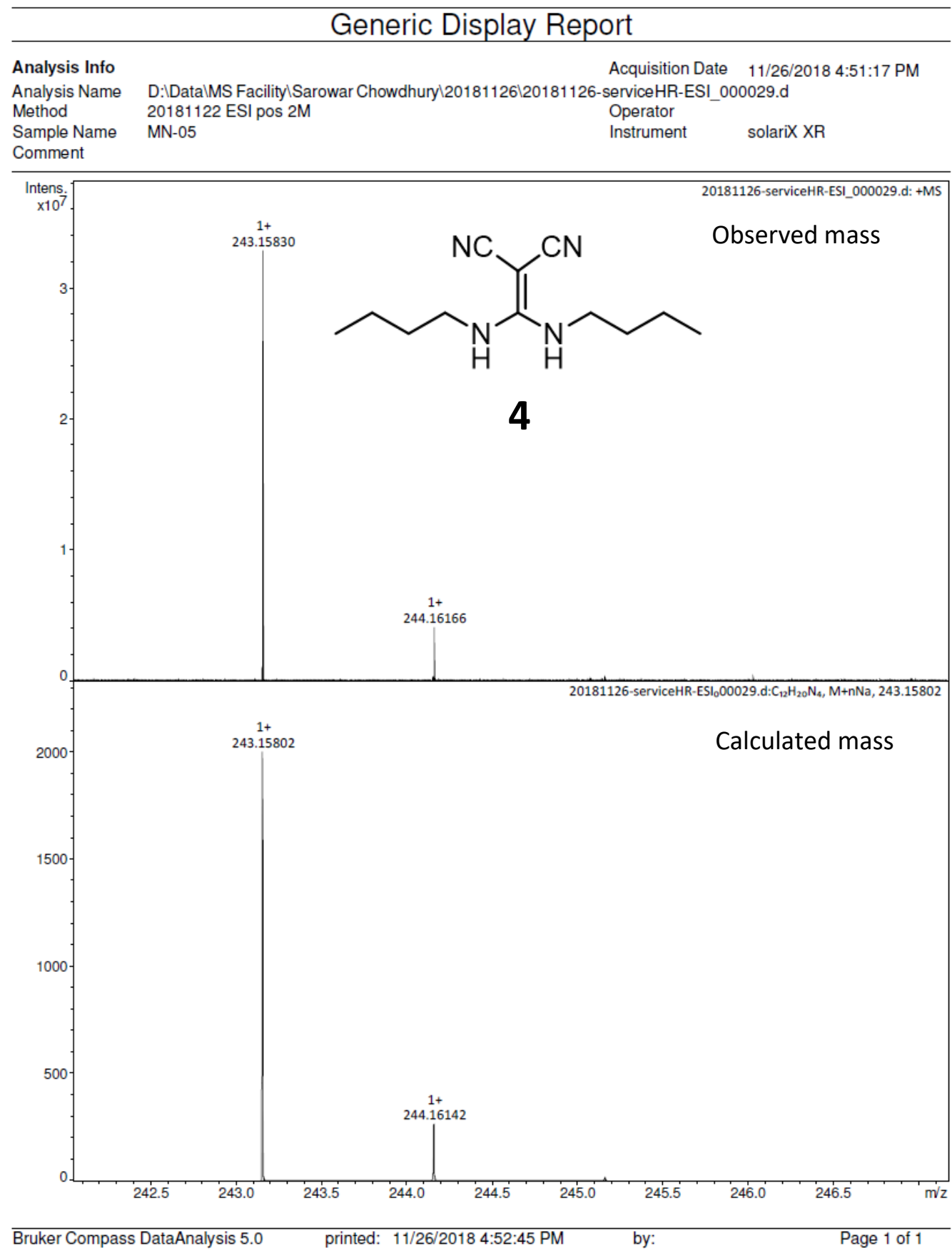




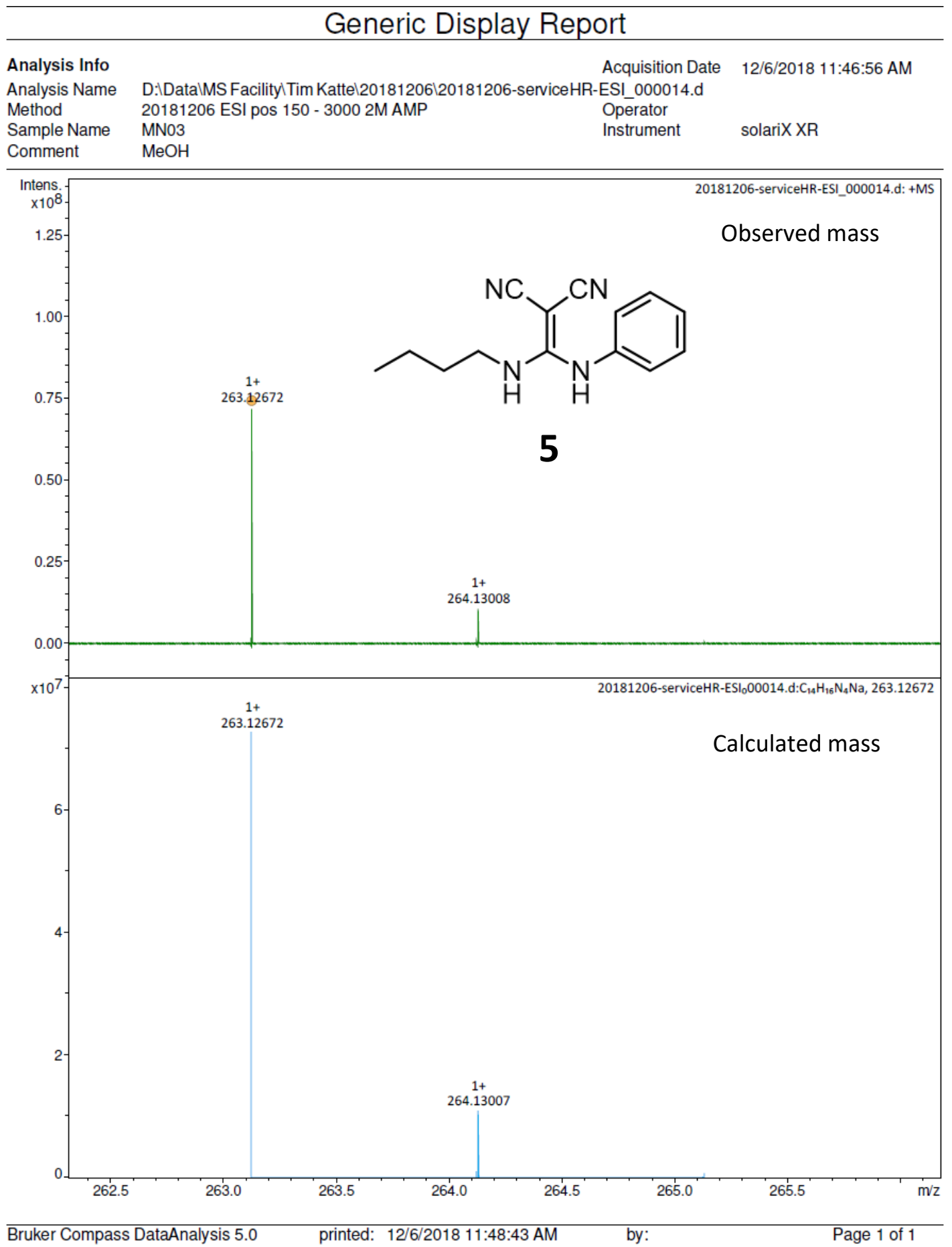




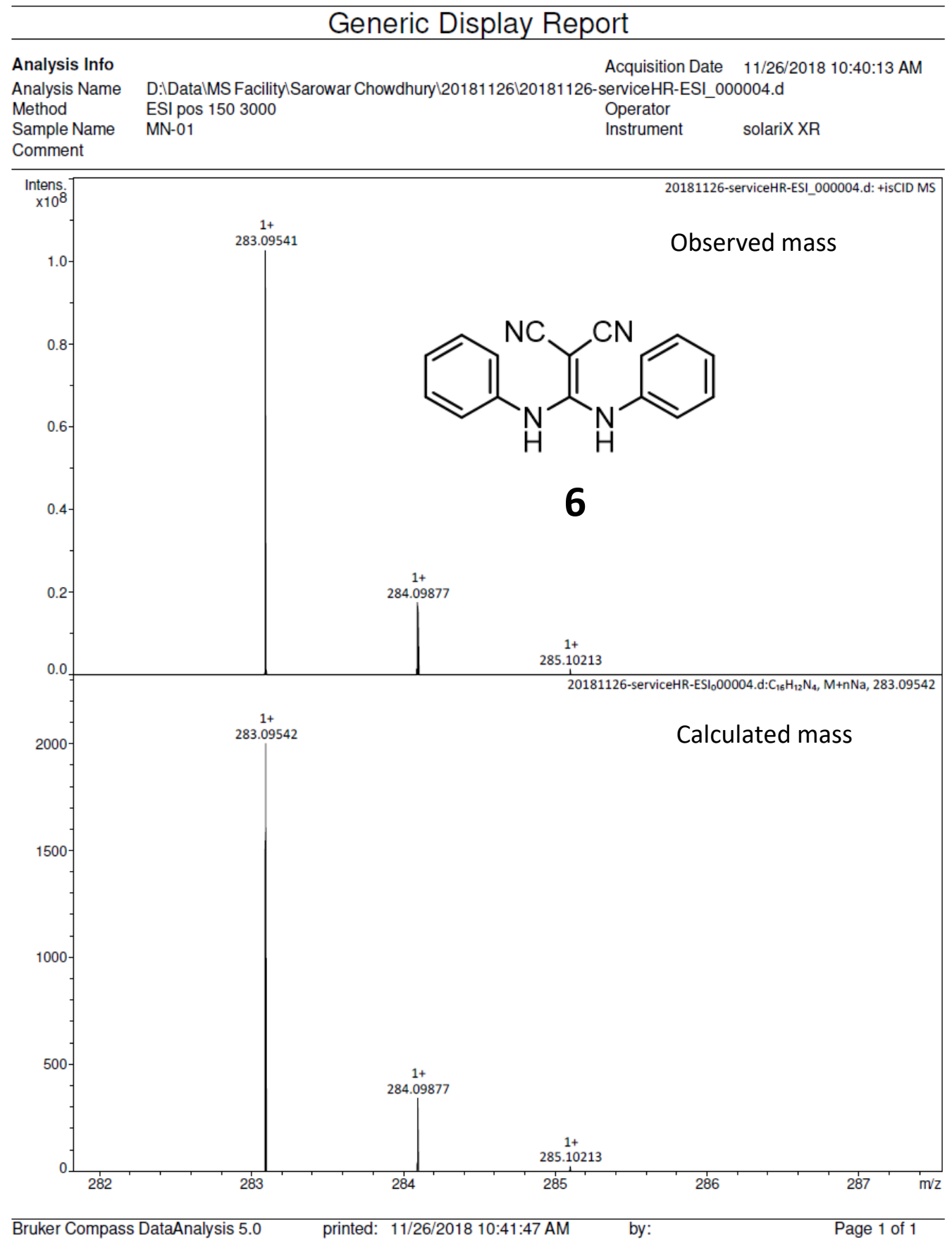




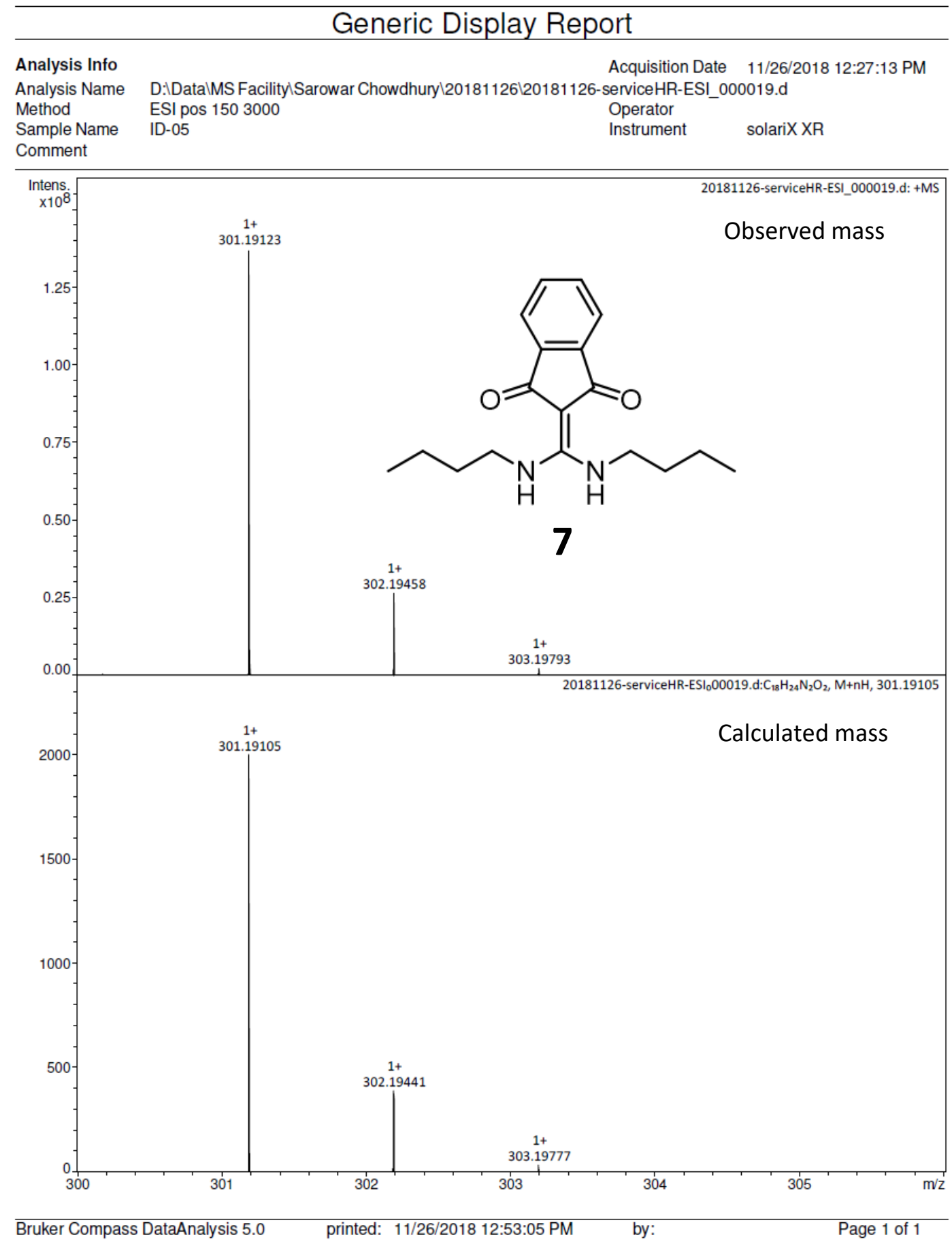




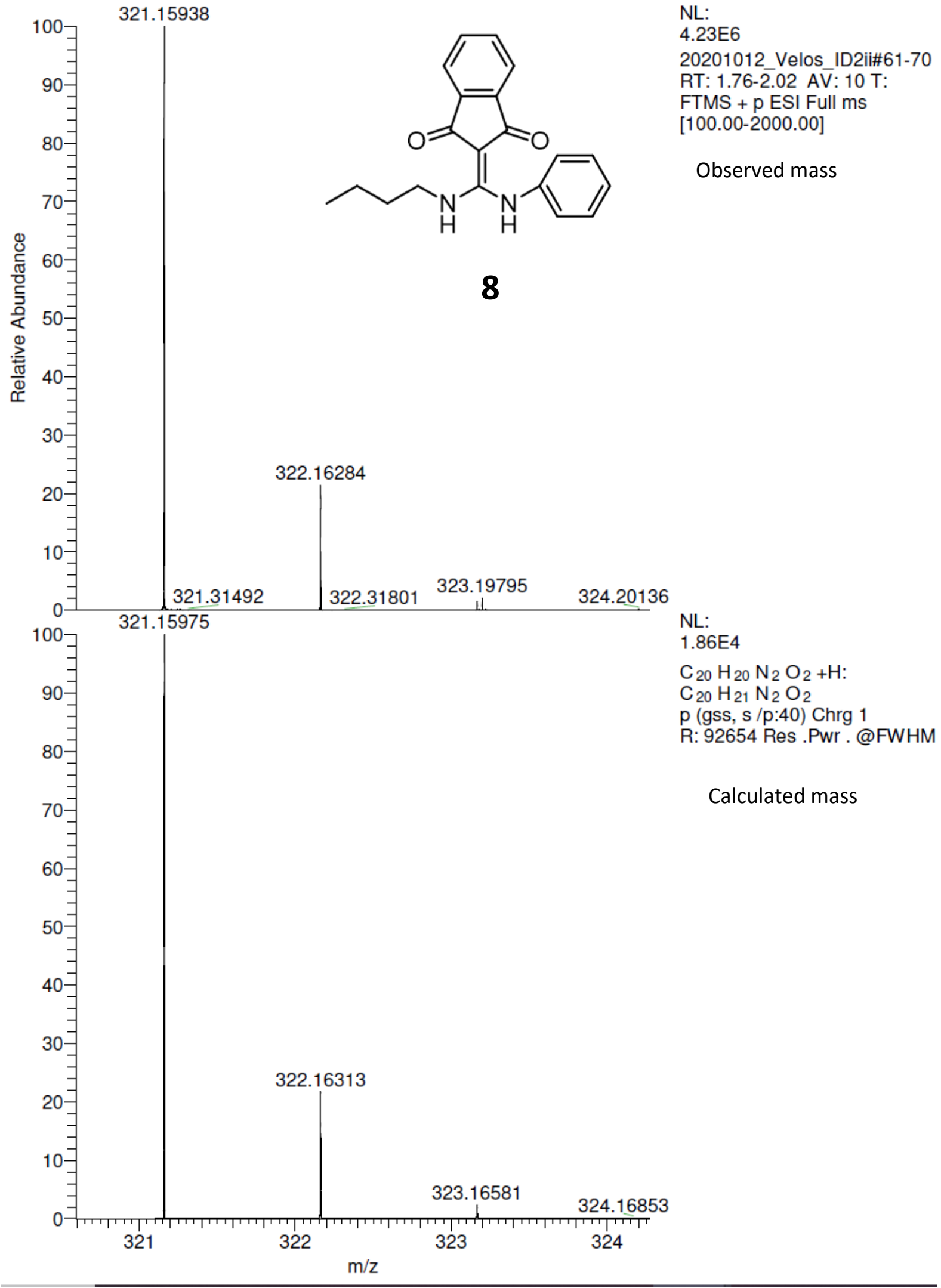




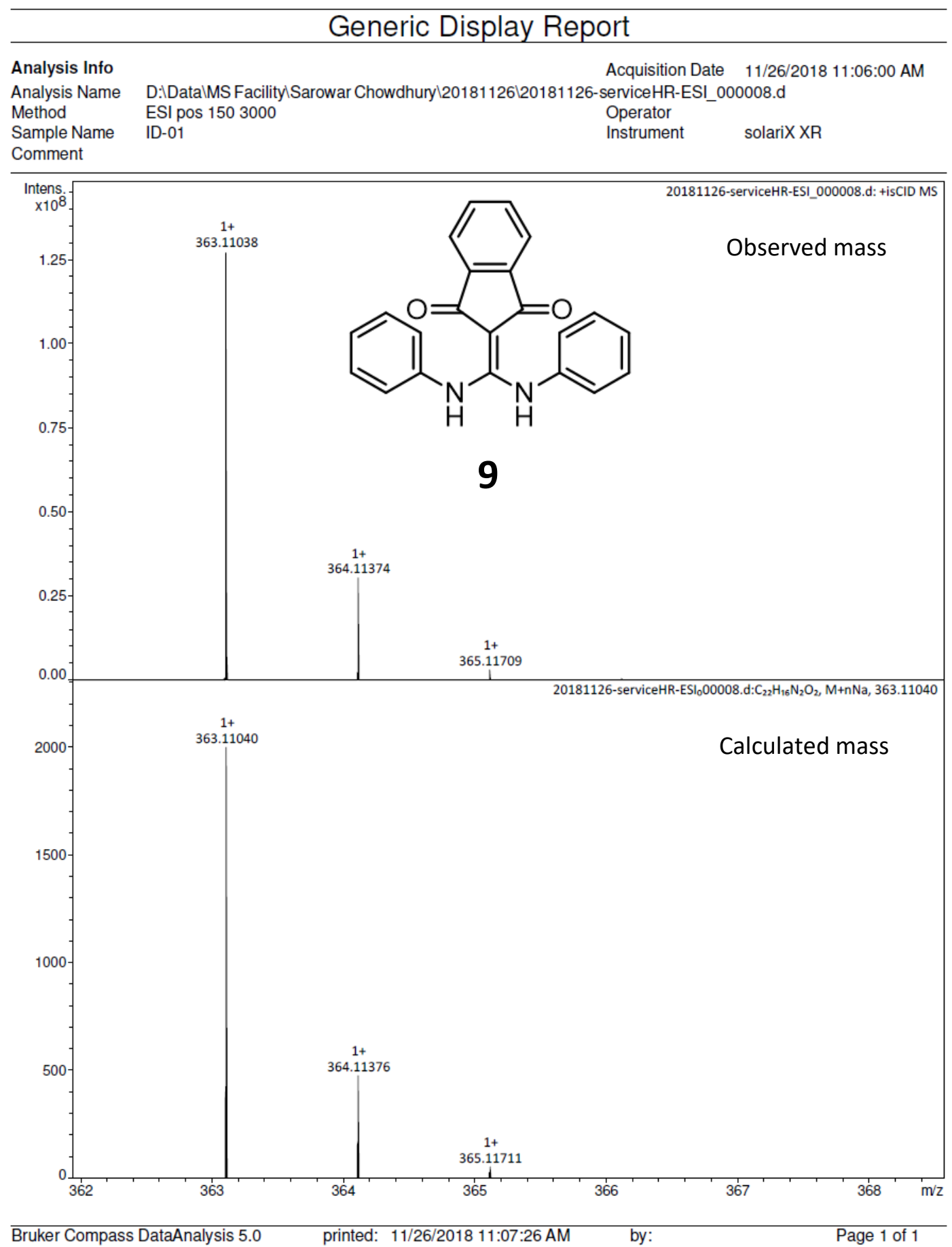




\section{Computational details}

All electronic structure calculations were performed using the Gaussian 16 program. ${ }^{2}$ Geometries and frequency calculations were obtained at the M06-2X/6-31G(d) level ${ }^{3}$ in conjunction with the SMD implicit solvent model ${ }^{4}$ to simulate solvent effects in acetonitrile. This level of theory was chosen for compatibility with the parameterisation of the SMD model. Systematic conformer searches were performed by generating a set of starting geometries corresponding to 2-fold or 3-fold rotation about each rotatable bond that were subsequently geometry-optimised (in SMD) and their ranked in terms of electronic energies. Scheme S1 illustrates this conformer generation process for compound $\mathbf{5}$ which results in a starting pool of 54 geometries.

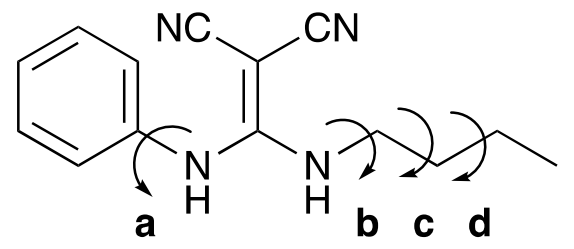

\begin{tabular}{cc} 
bond & $n$-fold rotation \\
\hline $\mathrm{a}$ & 2 \\
$\mathrm{~b}$ & 3 \\
$\mathrm{c}$ & 3 \\
$\mathrm{~d}$ & 3
\end{tabular}

Total $=2 \times 3 \times 3 \times 3=54$

Scheme S1. 
M06-2X/6-31G(d) + SMD (acetonitrile) optimised geometries. All optimised geometries have zero imaginary frequencies.

\begin{tabular}{|c|c|c|c|}
\hline & $\begin{array}{l}(\text { syn,anti) } \\
=-862.47651\end{array}$ & $10 \mathrm{H}$ & \\
\hline & -0.1607881842 & 0.8383482093 & -0.3225463735 \\
\hline & -1.0033330528 & 0.3883773407 & 0.62046 \\
\hline & 0.9533847002 & 0.1145768622 & -0.5230239922 \\
\hline & -0.4392975367 & 2.2611946918 & -1.2346001084 \\
\hline & -0.7774583591 & -0.4804630654 & 1.0912288276 \\
\hline & 1.5899233607 & 0.4663253292 & -1.2273322281 \\
\hline & 1.3154148725 & -1.104341 & 0.18762 \\
\hline & 4603 & -1.632 & -0.32 \\
\hline & 3.06 & -2.911 & 975 \\
\hline & 47745 & -3.444 & -0.1 \\
\hline & 55198 & -1.863 & 9785857 \\
\hline & 1.3 & -0.89 & \\
\hline & 3.41 & -0.859 & -0.1 \\
\hline & 99941 & -1.821 & -1.4 \\
\hline & 32461 & -3.673 & 0.2 \\
\hline & 3.13 & -2.71 & \\
\hline & 4.34 & -3.67 & -1 . \\
\hline & 4.6942014451 & -4.361 & 0.40 \\
\hline & 5.20 & -2.707 & 23 \\
\hline & -2.25 & 0.97 & \\
\hline & -3.4 & 0.44 & 17 \\
\hline & -3.6537626155 & -1.054460475 & 0.225 \\
\hline & $-4 \cdot 8 c$ & -1.55 & -0 \\
\hline & -2.2 & & \\
\hline & $-2.4 \xi$ & 0.7 & \\
\hline & -3.219251675 & 0.6973 & -0.96 \\
\hline & -4.34 & 0.98 & 0 . \\
\hline & -3.85 & -1.25 & \\
\hline & -2.7 & -1.58 & -0 \\
\hline & $-5 \cdot 7<$ & -1.05 & -0 \\
\hline & $-4 \cdot 95$ & -2.63 & -0 \\
\hline & $-4 \cdot 61$ & $-1 \cdot 34$ & -1 \\
\hline \multicolumn{4}{|c|}{$(\operatorname{ant} i, \operatorname{ant} i)$} \\
\hline & \multicolumn{3}{|c|}{$=-862.4754333720$ Hartree } \\
\hline & 0.19 & 2.479 & -0 \\
\hline & -0.94 & & -0 \\
\hline & 1.20 & $1.68 \mathrm{r} \cdot \mathrm{r} \mathrm{r} \mathrm{r} \mathrm{r} \mathrm{r} \mathrm{c}$ & 35 \\
\hline & 0.35 & 4.17 & -0 \\
\hline $\mathrm{H}$ & -0.9757194772 & 0.83 & -0.2 \\
\hline $\mathrm{H}$ & 1.0 & & 03 \\
\hline & 82988 & 2.113 & 0.5 \\
\hline & 3.46 & 2.17 & -0.65 \\
\hline $\mathrm{C}$ & 4.87 & 2.62 & -0.2 \\
\hline C & 5.7 & & -1 \\
\hline & 20298 & 1.40 & 584 \\
\hline & 2.5 & 3.095 & 1.0 \\
\hline & 3.0 & & -1 \\
\hline $\mathrm{H}$ & 3.4 & & -1 \\
\hline $\mathrm{H}$ & 5.2968255698 & 1.9402504532 & 22634 \\
\hline & 4.8 & & \\
\hline & 5.88 & 1.687 & -1.97 \\
\hline $\mathrm{H}$ & 6.8074179676 & 3.001 & -1.23 \\
\hline $\mathrm{H}$ & 5.413 & 3.3704797544 & -2.2605086693 \\
\hline & -2.19 & & -0 . \\
\hline C & -3.0630341442 & & 0.3405 \\
\hline & -4.371 & 3.511 & 0518718 \\
\hline
\end{tabular}




$\begin{array}{llcl}\mathrm{C} & -5.250679857 & 3.9033007954 & 1.0862448721 \\ \mathrm{H}-2.7308035875 & 1.7451861486 & -1.4833173114 \\ \mathrm{H}-1.9651141277 & 3.3406590273 & -1.4611269468 \\ \mathrm{H}-2.494708894 & 3.5511389751 & 0.9746332926 \\ \mathrm{H}-3.2751552102 & 1.9664375006 & 0.9436427415 \\ \mathrm{H}-4.9197877391 & 2.8217393178 & -0.752921513 \\ \mathrm{H}-4.1463203133 & 4.4008309056 & -0.7010697903 \\ \mathrm{H}-5.510082727 & 3.0249061997 & 1.6876193848 \\ \mathrm{H}-6.1832425307 & 4.3713520601 & 0.7557893369 \\ \mathrm{H}-4.7313786545 & 4.6130047031 & 1.7397299603\end{array}$

\section{2 (syn, anti)}

$E=-936.2629385710$ Hartree

C $-0.19376148140 .652557359 \quad-0.5059702946$

$\begin{array}{llll}\mathrm{N}-1.3585822638 & 0.4224585798 & 0.1607525027\end{array}$

$\begin{array}{llll}\mathrm{N} & 0.9408039451 & 0.2608308386 & 0.0796495936\end{array}$

S - $0.2371020635 \quad 1.4213845192-2.0231722866$

C $-1.5522691178-0.2484632786 \quad 1.3939374648$

$\begin{array}{llll}\text { C }-2.4144160923 & 0.322018753 & 2.3345315826\end{array}$

C $-2.667245511-0.3368806234 \quad 3.5333593593$

C $-2.0518310639-1.5571687752 \quad 3.8102704604$

C $-1.1968071764-2.1258690531 \quad 2.8686483301$

C $-0.9542493102-1.4859090127 \quad 1.6555858327$

$\mathrm{H}-2.1654202528 \quad 0.8938525602 \quad-0.2319994956$

$\mathrm{H} \quad 0.8987544148 \quad-0.0558070302 \quad 1.0443004192$

$\mathrm{H}-2.879702926 \quad 1.2794265865 \quad 2.1173111688$

$\mathrm{H}-3.3409366932 \quad 0.1114848329 \quad 4.2571511107$

$\mathrm{H}-2.2436686433-2.0647239432 \quad 4.7502717343$

$\mathrm{H}-0.7267584133-3.0844273747 \quad 3.0662808175$

$\mathrm{H}-0.3241788636-1.9551499373 \quad 0.905589158$

C $2.2726502417 \quad 0.4494870101-0.4851525581$

$\begin{array}{llll}\text { C } & 3.3152374436 & -0.2114917652 & 0.4073043794\end{array}$

C $3.1549708387-1.7271758308 \quad 0.5234947641$

C $4.2704564975-2.3575244193 \quad 1.3519073777$

H $2.4758525517 \quad 1.5210486703-0.5931528826$

$\mathrm{H} \quad 2.2904424817 \quad 0.0096052072-1.4876281313$

$\mathrm{H} \quad 3.2821309931 \quad 0.2442299605 \quad 1.4064504957$

$\mathrm{H} \quad 4.3020075396 \quad 0.0196507516 \quad-0.0105094111$

$\mathrm{H} \quad 3.1442563874-2.1638176141-0.4834457408$

$\mathrm{H} \quad 2.1849402781-1.9672158582 \quad 0.9781283609$

H $\quad 5.2494889736-2.1647510635 \quad 0.899496658$

$\mathrm{H} \quad 4.1446244181-3.4415547239 \quad 1.4331209965$

$\mathrm{H} \quad 4.2847878677-1.9439013251 \quad 2.3664762338$

\section{2 (anti, anti)}

$\mathbf{E}=-936.2598071600$ Hartree

C $-0.2277556568 \quad 1.2145103072 \quad 0.1746869843$

$\mathrm{N}-1.2550535197 \quad 0.3468756632-0.0516453614$

$\mathrm{N} \quad 0.982714249 \quad 0.6748276651 \quad-0.040669284$

S - $0.4370293721 \quad 2.8260569501 \quad 0.6676725366$

$\begin{array}{llll}\text { C }-2.6471181845 & 0.5647434961 & 0.0817306775\end{array}$

C $-3.4787869745 \quad 0.0981996936-0.939479044$

C $-4.8604663579 \quad 0.2271854094 \quad-0.82880525$

C $-5.4202941948 \quad 0.8308162788 \quad 0.2951568629$

C $-4.5871721496 \quad 1.2876766275 \quad 1.3158543106$

C $-3.2054779586 \quad 1.1480179793 \quad 1.2221680685$

$\mathrm{H}-1.0142810702-0.509828235-0.5433617797$

$\mathrm{H} \quad 1.0233880218-0.2892262977-0.3578062939$

$\mathrm{H}-3.034792593-0.3630872337-1.8175957638$

$\mathrm{H}-5.4971024049-0.1399891478-1.6280662322$

$\mathrm{H}-6.4972383297 \quad 0.9381866139 \quad 0.3794065279$

$\mathrm{H}-5.0146592227 \quad 1.7452245412 \quad 2.2030550921$ 


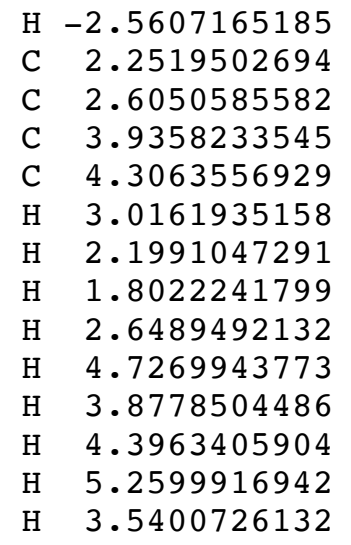

1.4813851861

1.3851103498

2.0776943661

$2.8207636017-1.2074700384$

$3.5055274765-2.519692787$

$0.6448455983 \quad 0.2713259933$

$2.1134278367 \quad 0.8292577732$

$2.7803006287-1.5534863317$

$1.3284262405-2.098585849$

$2.1154475944-0.9216595915$

$3.5667155407-0.4043648398$

$2.773888109-3.33028959$

$4.0365259034-2.4390819667$

$4.2325952568-2.8104699413$

\section{3 (syn, anti)}

$E=-1010.0462753600$ Hartree

C $-0.2586557355 \quad 1.5583504905$

$\mathrm{N}-1.4611100487 \quad 1.4225367562$

N 0.7656487574

0.0864016929

S -0.1472582687

$87960334 \quad 0.648351522$

C -1.7813978628

2.5011495499

C -1.7813978628

0.7537918098

$-1.3172382981$

C -3.3154311822

$-0.032559315$

1.9109884098

C -2.5408817488

$-0.6581527944$

1.9431927787

C -1.3929705173

$-0.5165495911$

3.1263181395

C -1.0141120749

C 2.1292476361

0.2722485928

4.2770437778

0.9195987679

4.2420302955

3.0686388272

C 2.7802045585

C 4.1358807698

0.8705775979

0.2600514858

$-0.3628439397$

0.1989683849

C 4.8431940867

$-0.4175975484$

$-0.113857979$

C 4.188877454

$0.7541141793-0.3730534248$

C 2.8376350497

$1.9838389331-0.3014348988$

$\mathrm{H}-2.2438268789$

2.0506777176

0.0241736899

H 0.5175984934

1.7856795273

0.1673739976

$\mathrm{H}-3.5279294164$

0.1575919163

1.3217464581

H -4.2153187425

$-0.1455928044$

1.039175223

$\mathrm{H}-2.8343665411$

$-1.2651932399$

3.1447306661

$-1.0122673699$

5.1969497216

H -0.7922995031

0.402128643

5.1369841129

H -0.1404662353

1.5640842588

3.0566386221

H 2.2190020851

$-1.2724611706 \quad 0.3948977675$

H $\quad 4.6352741834-1.3804991984-0.1595036371$

H $\quad 5.898763296 \quad 0.7113928643 \quad-0.6225438038$

$\mathrm{H} \quad 4.7360221045 \quad 2.903131743 \quad-0.4871415068$

$\begin{array}{llll}\mathrm{H} & 2.3337327268 & 3.0068215908 & 0.1014069301\end{array}$

\section{3 (anti, anti)}

$E=-1010.0430998500$ Hartree

C -0.2473910421

$\mathrm{N}-1.3652452482$

0.9586215199

0.3719919801

$-0.0000207433$

N 0.8921853739

S -0.2710959951

0.4153146419

0.5013281246

0.501282827

C -2.7205584044

$2.1954330692-1.1507281465$

C -3.5628612986

0.6533795336

0.1892284847

C -4.9156779317

$-0.4240018875-0.0911464865$

C -5.4298307409

$-0.2037529419-0.3375401702$

C -4.5854336513

$1.0906248996-0.314718568$

C -3.2355868845

$2.1626026447-0.0258869795$

C 2.2356196969

$\begin{array}{ll}1.9507482717 & 0.2374421871\end{array}$

C 3.1196187065

$0.7497921697 \quad 0.1902536409$

C 4.4629127652

$-0.2934458965-0.0908364932$

C 4.9259573811

$-0.0200814394-0.3361642038$

$1.2934120373-0.311574636$ 


$\begin{array}{cccc}\mathrm{C} & 4.0400530184 & 2.3311684533 & -0.0220637332 \\ \mathrm{C} & 2.6994053248 & 2.0662859711 & 0.2402146632 \\ \mathrm{H} & -1.2338178121 & -0.4696139456 & 1.056899588 \\ \mathrm{H} & 0.7933794968 & -0.4313946389 & 1.0558744174 \\ \mathrm{H} & -3.1518858758 & -1.4295804274 & -0.1157604954 \\ \mathrm{H} & -5.5639726032 & -1.0473055333 & -0.554032419 \\ \mathrm{H} & -6.4828484691 & 1.2637756784 & -0.5137921389 \\ \mathrm{H} & -4.981217692 & 3.1731541284 & 0.0083853675 \\ \mathrm{H} & -2.5828674631 & 2.7796102429 & 0.4836189767 \\ \mathrm{H} & 2.7483621619 & -1.3143203627 & -0.1168335908 \\ \mathrm{H} & 5.1438797728 & -0.8373398995 & -0.5532092217 \\ \mathrm{H} & 5.9714968721 & 1.5079055101 & -0.509810176 \\ \mathrm{H} & 4.3959266277 & 3.3564090355 & 0.0135780042 \\ \mathrm{H} & 2.0145639141 & 2.8686571852 & 0.4869219208\end{array}$

\section{4 (syn, anti)}

\section{$E=-688.0243974620$ Hartree}

C $0.3961818694 \quad 2.0153311682 \quad-0.4668497582$

$\mathrm{N}-0.9040936286 \quad 1.7088793539-0.4350337667$

$\mathrm{N} \quad 1.2704368673 \quad 1.0124170722-0.62897662$

C $0.9002354833 \quad 3.3504894268 \quad-0.3695725698$

$\mathrm{H}-1.1385500551 \quad 0.7281457135-0.3261287938$

$\mathrm{H} \quad 2.2459237881 \quad 1.2086732972 \quad-0.4323646878$

C $\quad 0.9269584372-0.3675851521-0.9572061638$

C $2.1654976686-1.1009973186-1.4579810526$

C $2.7338153725-0.520852437-2.7524995677$

C $3.9463178272-1.3054903861-3.2439689573$

$\mathrm{H} \quad 0.1574229268-0.3565691073-1.7381567577$

$\mathrm{H} \quad 0.5115840524-0.8750949852 \quad-0.0773673691$

H $\quad 1.8908711647-2.1505957098-1.612631606$

$\mathrm{H} \quad 2.9334869182-1.0878553206 \quad-0.67279851$

$\mathrm{H} \quad 3.0136485449 \quad 0.5289677428 \quad-2.5987108598$

$\mathrm{H} \quad 1.9491641767-0.5257169685-3.5199231137$

H $\quad 4.7485876981-1.292309245-2.4978669515$

$\mathrm{H} \quad 4.3442948185 \quad-0.8844739466-4.1722784977$

H $\quad 3.6842665225 \quad-2.3522584676 \quad-3.4337492017$

C $-2.0383979942 \quad 2.6190485886 \quad-0.5668029594$

C $-2.7150722203 \quad 2.9287412862 \quad 0.7656185085$

$\begin{array}{llll}\text { C }-3.7984871073 & 3.9923904952 & 0.6065633751\end{array}$

C $-4.5034303799 \quad 4.2992240667 \quad 1.9241395567$

$\mathrm{H}-2.7517409914 \quad 2.1401906492-1.2448644707$

$\mathrm{H}-1.6950745065 \quad 3.5321226404-1.0578898134$

$\mathrm{H}-1.9630819652 \quad 3.273582682 \quad 1.4842566411$

$\mathrm{H}-3.1494271187 \quad 2.0053566654 \quad 1.1702372073$

$\mathrm{H}-4.5314506249 \quad 3.6600339272-0.1398545367$

$\mathrm{H}-3.3389274016 \quad 4.9075875832 \quad 0.2107293337$

$\mathrm{H}-4.9847526766 \quad 3.40217822 \quad 2.3291356711$

$\mathrm{H}-5.2739232726 \quad 5.0658501568 \quad 1.7964691908$

$\mathrm{H}-3.7887954786 \quad 4.6615140522 \quad 2.6715057152$

C $\quad 0.1841435774 \quad 4.4237666055 \quad 0.2012524497$

C $2.24060004643 .5935539833-0.7454049309$

$\mathrm{N}-0.3865660796 \quad 5.3206941861 \quad 0.6761899801$

$\mathrm{N} \quad 3.3467467409 \quad 3.7662404817 \quad-1.0644881134$

\section{4 (anti, anti)}

$E=-688.0214383600$ Hartree

C $0.2512667178 \quad 2.5466616716 \quad-0.1347096844$

$\begin{array}{llll}\mathrm{N} & -0.9374495498 & 1.9630907638 & 0.0815279976\end{array}$

$\mathrm{N} \quad 1.3140066001 \quad 1.7307792073-0.206569558$

C $0.3784420466 \quad 3.9610282581-0.2804524738$

$\mathrm{H}-0.9094426636 \quad 0.9916264062 \quad 0.3767520094$

$\mathrm{H} \quad 1.1094128479 \quad 0.7402122257 \quad-0.2993060705$

C $2.7159061552 \quad 2.0642020657 \quad 0.0351913426$ 
C 3.5752852592

C 5.0001504449

C 5.8884160763

$\mathrm{H} \quad 3.0848339653$

$\mathrm{H} \quad 2.7666707286$

H 3.1205571373

H 3.5888335482

$\mathrm{H} \quad 5.4340130518$

$\mathrm{H} \quad 4.9638214581$

$\mathrm{H} \quad 5.9587085339$

$\mathrm{H} \quad 6.9028651227$

$\mathrm{H} \quad 5.4822520435$

C -2.262460967

C -3.1087687555

C -4.4291691235

C -5.3107936756

$\mathrm{H}-2.7591716507$

$\mathrm{H}-2.1412064302$

$\mathrm{H}-2.5448445418$

$\mathrm{H}-3.3014794949$

$\mathrm{H}-4.9671853962$

$\mathrm{H}-4.2121176714$

$\mathrm{H}-5.5602880928$

$\mathrm{H}-6.2482182465$

$\mathrm{H}-4.7977770873$

C -0.5668775912

C 1.455812653

$\mathrm{N}-1.3341526888$

N 2.3334352362
$1.9384716339-1.2191779737$

$2.4277016935-0.9721071356$

$2.2647391616-2.201568089$

$1.3827718048 \quad 0.8093509923$

$3.0743200148 \quad 0.4466099484$

$2.5194550068-2.0294263572$

$0.8889136215-1.5411427818$

$1.8812021255-0.1247433051$

$3.4851668933-0.6787714149$

$1.2120456387-2.4969799196$

$2.6296129887-2.0132392921$

$2.8239859554-3.0517221996$

$2.4825820871-0.2493477493$

$2.766523548 \quad 0.9879444825$

$3.4386933279 \quad 0.6200484568$

$3.686942401 \quad 1.8399930369$

$1.734698322-0.8768397684$

$3.381469408-0.8571875353$

$3.4101029044 \quad 1.6725110836$

$1.8229336623 \quad 1.5151285977$

$2.8171346695-0.1073591946$

$4.39164001 \quad 0.1196656455$

$2.7450083185 \quad 2.3408788695$

$4.1788459808 \quad 1.5625553953$

$4.3263252745 \quad 2.5669661891$

$4.851906099 \quad 0.2756114452$

$4.5400677226-0.9878981835$

$5.5941908925 \quad 0.7395770566$

$5.0259992351-1.5783248626$

\section{5 (syn, syn)}

\section{$E=-761.8104711540$ Hartree}

C $0.707597187 \quad 1.5570192942$

$\begin{array}{llll}\mathrm{N} & -0.445857148 & 0.8531446354 & 0.2537330624\end{array}$

$\begin{array}{lllll}\mathrm{N} & 1.8763870761 & 0.9220259543 & 0.0454335265\end{array}$

C $0.6586402578 \quad 2.9729086932 \quad 0.2079874318$

$\mathrm{H}-1.2952426349 \quad 1.3694554719 \quad 0.0442859887$

$\begin{array}{llll}\mathrm{H} & 2.7078654254 & 1.4942065157 & 0.1630804872\end{array}$

$\begin{array}{llll}\text { C }-0.6440138486 & -0.437893491 & 0.8075095308\end{array}$

$\begin{array}{llll}\text { C }-0.5072529854 & 3.6478055472 & 0.6353768386\end{array}$

C $1.7808301778 \quad 3.7246705865-0.2095610995$

C $0.1850262132-0.9492007106 \quad 1.8086796523$

C $-0.0796757455-2.2101142197 \quad 2.3368966495$

C $-1.1715706811-2.952844483 \quad 1.8920096689$

C $-2.005189019-2.425919998 \quad 0.9066600644$

C $-1.742444919-1.1745844094 \quad 0.3589555968$

H $\quad 1.0228340909-0.3649524085 \quad 2.1760963849$

$\mathrm{H} \quad 0.5690952558-2.6051154351 \quad 3.1126689891$

$\mathrm{H}-1.3734110501-3.9327824136 \quad 2.3126039913$

$\mathrm{H}-2.862192481-2.9920405962 \quad 0.5550076474$

$\mathrm{H}-2.3814768488-0.760666364-0.4161743506$

$\begin{array}{llll}\mathrm{N} & -1.4768903944 & 4.187679684 & 0.9826548594\end{array}$

$\begin{array}{llllll}\mathrm{N} & 2.7238750328 & 4.3091448116 & -0.5589156028\end{array}$

C $2.0947176192-0.3851570705-0.5780823618$

C $3.2581711795-0.3015340884-1.5610471043$

C $3.01369383820 .666286416 \quad-2.7170522407$

C $4.1666509823 \quad 0.66712063-3.7162187218$

H $\quad 1.1761690832-0.6706097158-1.0988133143$

$\mathrm{H} \quad 2.2936811472-1.1367362832 \quad 0.1932582553$

$\mathrm{H} \quad 3.4329315741-1.3113305859-1.9510793988$

$\mathrm{H} \quad 4.1683030968-0.0119959194 \quad-1.0180768371$

H $2.8704244207 \quad 1.6809134079 \quad-2.3240609571$ 


\begin{tabular}{|c|c|c|c|}
\hline & 2.079565509 & .3911712893 & -3.2237911818 \\
\hline & 6167 & 59475 & -3.22 \\
\hline & 838648844 & 3697953268 & 977 \\
\hline & & -0.3 & \\
\hline & \multicolumn{3}{|l|}{$($ anti, anti) } \\
\hline & & & \\
\hline & 403 & 1.96 & 0 \\
\hline & -0.71 & & \\
\hline & 074 & 368 & -0 \\
\hline & 4824 & 3.27612 & 428 \\
\hline & 01 & 0.0 & 0.2 \\
\hline & 37 & & -0 . \\
\hline & -2.11 & 508 & 0 . \\
\hline & -1.21 & 3.5189285233 & 0895 \\
\hline & 51 & 4.4 & 0 . \\
\hline & -2 & 51 & -0 . \\
\hline & -4 & 268 & -1 \\
\hline & $-4.87 \varepsilon$ & 485 & -0.2 \\
\hline & -4 & 0.40 & 0.5 \\
\hline & -2 & & 0.74 \\
\hline & 562 & 911 & 2886 \\
\hline & -4.42 & 2.93 & 36281291 \\
\hline & -5 & 1.4 & -0 . \\
\hline & -5 . & -0 & 87 \\
\hline 音 & -2 . & -0 & 26264 \\
\hline & -2.1319887034 & 3.728773 & 61913055 \\
\hline & 8203 & 5.33 & 286593 \\
\hline 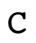 & 2 & 2. & 4067 \\
\hline & 3.3624 & 2.893527199 & 5947564 \\
\hline & 4.6166203183 & 3.6773123614 & -0.6905634817 \\
\hline & 5.292 & 4.30 & 47980527 \\
\hline & 3.3 & 1.4 & 6276172 \\
\hline $\mathrm{H}$ & 2.5581385303 & 2.9652979538 & 0.9495239211 \\
\hline & 2.636335218 & 3.5643450577 & -1.5423616304 \\
\hline & 3.6118 & 2.1 & 9863112 \\
\hline$H$ & & 3.0128325191 & -0.1750420576 \\
\hline $\mathrm{H}$ & 4.3401107795 & 4.4604725907 & 0.0275287286 \\
\hline & 5.595486549 & 3.5395934087 & -2.6256006252 \\
\hline & 52604 & 58 & 60663 \\
\hline & 4.6098440051 & 4.9949019484 & -2.416276696 \\
\hline
\end{tabular}
6 (syn, syn)
$E=-835.5964216200$ Hartree
C $\quad 0.3927491811 \quad 1.2069986205 \quad 0.2473966582$
$\mathrm{N}-0.8152904674 \quad 0.6993270502 \quad 0.5722734485$
$\begin{array}{lllll}\mathrm{N} & 1.466874704 & 0.4028884603 & 0.0980760377\end{array}$
C $\quad 0.5348241999 \quad 2.5987155336 \quad 0.0611465765$
$\mathrm{H}-1.6161283728 \quad 1.2909159357 \quad 0.3691320431$
$\mathrm{H} \quad 2.374336016 \quad 0.8557500199 \quad 0.1614446405$
C $-1.1053396056-0.5029057165 \quad 1.2669061907$
$\begin{array}{llll}\text { C }-0.4781047147 & 3.4891066192 & 0.4898119855\end{array}$
C $\quad 1.6926169461 \quad 3.1273570514 \quad-0.5574238952$
C $-0.245724879-1.0166599582 \quad 2.2397468385$
$\begin{array}{llll}C & -0.5787437878 & -2.204588847 & 2.8833779246\end{array}$
C $-1.7685804835-2.8663000333 \quad 2.582714181$
C $-2.6327022173-2.3311309656 \quad 1.6292359201$
C $-2.3015857414-1.1545778778 \quad 0.9643709581$
$\mathrm{H} \quad 0.6734321169-0.4951032093 \quad 2.4877537265$
$\mathrm{H} \quad 0.0947569982 \quad-2.6077696102 \quad 3.6333875709$
$\mathrm{H}-2.0220885347-3.7904568367 \quad 3.0922399963$
$\mathrm{H}-3.5646330459-2.8343849624 \quad 1.3910662384$
$\mathrm{H}-2.9591323921 \quad-0.7391524541 \quad 0.2056042804$ 


$\begin{array}{lrll}\mathrm{C} & 1.4938907813 & -0.9714871925 & -0.2517366849 \\ \mathrm{C} & 2.5445371987 & -1.746018017 & 0.2416729965 \\ \mathrm{C} & 0.5238550276 & -1.5394703999 & -1.0798223514 \\ \mathrm{C} & 0.5971665532 & -2.8955868081 & -1.3829912061 \\ \mathrm{C} & 1.6394898419 & -3.6788181326 & -0.8889441693 \\ \mathrm{C} & 2.6176792025 & -3.0965345806 & -0.0850011055 \\ \mathrm{H} & 3.2913921375 & -1.2865448863 & 0.8833620741 \\ \mathrm{H} & -0.279703307 & -0.9287079617 & -1.4793739474 \\ \mathrm{H} & -0.1626547201 & -3.3380428593 & -2.0198403163 \\ \mathrm{H} & 1.6908356826 & -4.7350051484 & -1.133446279 \\ \mathrm{H} & 3.4371261726 & -3.6950993826 & 0.3006299163 \\ \mathrm{~N} & -1.3224293682 & 4.2062576901 & 0.8413644433 \\ \mathrm{~N} & 2.6528828775 & 3.5458508592 & -1.0609426906\end{array}$

6 (anti, anti)

$E=-835.5926440170$ Hartree

$\begin{array}{llll}\text { C } & 0.3995375059 & 1.2735227868 & 0.2385172461\end{array}$

$\begin{array}{llll}\mathrm{N} & -0.7830820935 & 0.7000014209 & 0.5515969247\end{array}$

$\begin{array}{llll}\mathrm{N} & 1.4359848876 & 0.415288712 & 0.1170633209\end{array}$

C $\quad 0.541475617 \quad 2.6639092511 \quad 0.0524104844$

$\mathrm{H}-0.7320326254 \quad-0.2072204551 \quad 1.0076438851$

$\mathrm{H} \quad 1.1937041715-0.550507288-0.0883011708$

$\begin{array}{llll}\text { C }-2.0904764371 & 1.1857285191 & 0.2820368772\end{array}$

$\begin{array}{llll}\text { C }-0.3970180343 & 3.5784503806 & 0.5900131865\end{array}$

C $1.6282202315 \quad 3.2017630812-0.6796226521$

C $-2.3985959639 \quad 1.819565751-0.923377405$

C $-3.6998356124 \quad 2.2559759223-1.1510131969$

C $-4.6962014962 \quad 2.0427679318-0.1990378536$

$\begin{array}{llll}\text { C }-4.3840206832 & 1.3891658251 & 0.9912639323\end{array}$

C $-3.0814235277 \quad 0.9655247427 \quad 1.2385833229$

$\mathrm{H}-1.6275736637 \quad 1.9692684595-1.6726562914$

$\mathrm{H}-3.936465505 \quad 2.7542689516 \quad-2.0860623864$

$\mathrm{H}-5.710417968 \quad 2.3808786059 \quad-0.3863930836$

$\mathrm{H}-5.1529335313 \quad 1.214190335 \quad 1.7373559068$

$\mathrm{H}-2.8244830087 \quad 0.4683170128 \quad 2.1696065469$

$\begin{array}{llll}\text { C } & 2.8204864815 & 0.6847827165 & 0.2855966977\end{array}$

C $3.7222143967 \quad 0.0309240807-0.5539290555$

C $3.2802404233 \quad 1.5362594276 \quad 1.2922386386$

$\begin{array}{llll}\text { C } & 4.647806762 & 1.7494444819 & 1.4329860222\end{array}$

$\begin{array}{lllr}\text { C } & 5.5563529151 & 1.1023160659 & 0.5961892947\end{array}$

C $5.08909755420 .2369013038-0.390826062$

$\mathrm{H} \quad 3.3470246268-0.6302000464-1.3299354836$

H $2.574477536 \quad 2.0258694061 \quad 1.9559292962$

$\begin{array}{llll}\mathrm{H} & 5.0034219636 & 2.4166703801 & 2.2120586328\end{array}$

$\begin{array}{llll}\mathrm{H} & 6.6221250441 & 1.2691388907 & 0.7160047857\end{array}$

$\mathrm{H} \quad 5.7878607725-0.2753419766-1.0449159272$

$\begin{array}{llll}\mathrm{N} & -1.1563738894 & 4.3367047659 & 1.0356766225\end{array}$

N 2.5109531502 3.6514505571 -1.286871056

\section{7 (syn, syn)}

$E=-959.9643526000$ Hartree

C $0.2002302468 \quad 1.4215020167 \quad-0.04729061$

$\begin{array}{llll}\mathrm{N} & -1.0015505212 & 0.9145449537 & 0.2619425103\end{array}$

$\mathrm{N} \quad 1.2541531924 \quad 0.631286846 \quad-0.295717388$

C $0.3612232154 \quad 2.8338197973 \quad-0.1133939532$

H $-1.7111005663 \quad 1.6335962224 \quad 0.4072075934$

H $\quad 2.1055145786 \quad 1.1560510567 \quad-0.4992137994$

C $1.2059119216-0.7379716006-0.8167447756$

C $2.2563837869-0.9107139481-1.9096028337$

C $2.0330182101-0.0076611194-3.1218344534$

C $3.0606012947-0.2616449558-4.2206818525$

H $\quad 0.2081043945-0.9173305423-1.229557252$

H $\quad 1.3757508396-1.4611638384-0.0126637$ 


$\begin{array}{cccc}\mathrm{H} & 2.2417776267 & -1.9615597403 & -2.2225498134 \\ \mathrm{H} & 3.252062451 & -0.7225813822 & -1.4846229337 \\ \mathrm{H} & 2.0818365619 & 1.0436004154 & -2.8115522216 \\ \mathrm{H} & 1.0203341127 & -0.1746980231 & -3.5113803367 \\ \mathrm{H} & 4.0771369245 & -0.083119552 & -3.8526713611 \\ \mathrm{H} & 2.8955626363 & 0.3942897179 & -5.0809935358 \\ \mathrm{H} & 3.0105431096 & -1.2980251056 & -4.5725871787 \\ \mathrm{C} & -1.2565752441 & -0.3764720144 & 0.9076239988 \\ \mathrm{C} & -2.3073385484 & -0.2072050153 & 2.0007459673 \\ \mathrm{C} & -1.8739968063 & 0.7305472921 & 3.1266843681 \\ \mathrm{C} & -2.9206178181 & 0.8163314567 & 4.2335393422 \\ \mathrm{H} & -0.3202258979 & -0.7364949476 & 1.3456242156 \\ \mathrm{H} & -1.5930589167 & -1.1140870636 & 0.1720286093 \\ \mathrm{H} & -2.5257810006 & -1.2009914635 & 2.409523588 \\ \mathrm{H} & -3.239787168 & 0.1596871105 & 1.5498526032 \\ \mathrm{H} & -1.688795782 & 1.7326794073 & 2.7201888668 \\ \mathrm{H} & -0.9205975389 & 0.3770432218 & 3.5404999499 \\ \mathrm{H} & -3.8749605093 & 1.1838343101 & 3.8400679637 \\ \mathrm{H} & -2.6033559676 & 1.4949676593 & 5.0312997843 \\ \mathrm{H} & -3.1008228709 & -0.1678072938 & 4.680109448 \\ \mathrm{C} & -0.6486920175 & 3.8008803646 & 0.2385229315 \\ \mathrm{C} & -0.0359619672 & 5.1587417354 & 0.0140632259 \\ \mathrm{C} & 1.2690175189 & 4.9882567582 & -0.450396408 \\ \mathrm{C} & 1.5587345187 & 3.5127202445 & -0.5423870469 \\ \mathrm{C} & -0.5758197285 & 6.4183034208 & 0.1991722479 \\ \mathrm{C} & 0.2338116345 & 7.5235160467 & -0.0962906206 \\ \mathrm{C} & 1.5384154191 & 7.3530538239 & -0.5611858961 \\ \mathrm{C} & 2.0759948481 & 6.0718462972 & -0.7450651548 \\ \mathrm{O} & -1.8027110119 & 3.612028967 & 0.6318723042 \\ \mathrm{O} & 2.636653077 & 3.0341049023 & -0.9045638994 \\ \mathrm{H} & -1.5920550285 & 6.5448576853 & 0.5608870611 \\ \mathrm{H} & -0.1578980371 & 8.5272641751 & 0.0381320341 \\ \mathrm{H} & 2.1441163836 & 8.2264230941 & -0.7833768272 \\ \mathrm{H} & 3.090836444 & 5.9330576067 & -1.1062057618\end{array}$

7 (anti, anti)

$E=-959.9522515260$ Hartree

C $\quad 0.1654415379 \quad 1.1158876393 \quad-0.0326778058$

$\begin{array}{llll}\mathrm{N} & -0.9469384928 & 0.5555241398 & 0.4671234592\end{array}$

N $\quad 1.1180784002 \quad 0.2761160559 \quad-0.4667915154$

C $0.326581799 \quad 2.5278384332-0.0988261253$

H $-0.9125157703-0.4442784104 \quad 0.6487157856$

H $\quad 0.8579019627 \quad-0.7027532119 \quad-0.5556246454$

$\begin{array}{llll}\text { C } & 2.5433650921 & 0.56325895 & -0.5945362822\end{array}$

$\begin{array}{llll}\text { C } 3.3695047486 & -0.5722786088 & 0.0018653416\end{array}$

$\begin{array}{llll}\text { C } & 3.1615359421 & -0.7579881345 & 1.5034997574\end{array}$

C $4.0405591617-1.8671112282 \quad 2.0736714762$

H $\quad 2.742935535 \quad 1.4983843677 \quad-0.0635147876$

H $2.7941432336 \quad 0.7235202511-1.6464818765$

$\mathrm{H} \quad 4.4260609965-0.3583171027-0.2008758168$

$\mathrm{H} \quad 3.134008651-1.5079549183-0.5245657683$

H $\quad 2.1070021471 \quad-0.9861894057 \quad 1.7030065964$

$\begin{array}{llll}\mathrm{H} & 3.3777272398 & 0.1892424087 & 2.0145821998\end{array}$

$\mathrm{H} \quad 3.8244672294 \quad-2.8253822064 \quad 1.5880925764$

$\mathrm{H} \quad 3.8800234021-1.9922212914 \quad 3.149002863$

H $5.1019223653-1.6458071941 \quad 1.9154392878$

$\begin{array}{llll}\text { C }-2.269792681 & 1.1662074188 & 0.5528082337\end{array}$

$\begin{array}{lll}\text { C }-3.3362744041 & 0.1952541793 & 0.0552974378\end{array}$

C $-3.1913268845-0.1703674979-1.4204928925$

C $-4.3031227705-1.1013825207-1.8948877549$

$\mathrm{H}-2.2597130097 \quad 2.0690753918-0.0644416996$

$\mathrm{H}-2.4669824216 \quad 1.4752523139 \quad 1.5827458179$ 


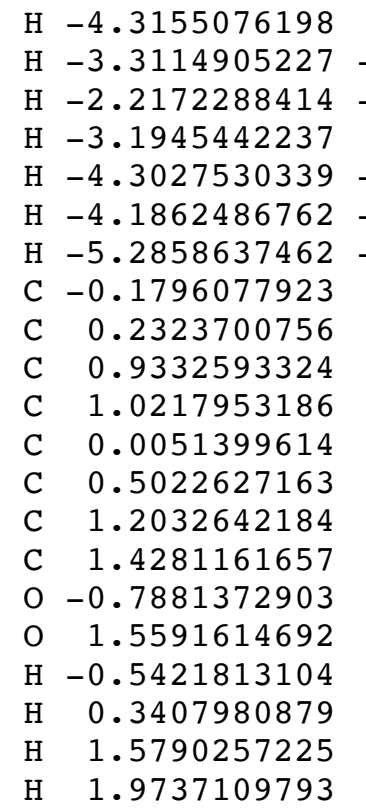

$0.6589784411 \quad 0.2263386783$

$-0.716663817 \quad 0.6683814243$

$-0.6471489375-1.5870256124$

$0.7498412309-2.0191542721$

$-2.0346373608-1.3206048933$

$-1.3581649221-2.9523209234$

$-0.6335713529-1.7688812417$

$3.4721275942 \quad 0.8790730518$

$\begin{array}{lll}4.8411329527 & 0.3938372658\end{array}$

$4.705127351-0.801874237$

$3.2397585144-1.154309167$

$6.0839784977 \quad 0.9587934625$

$7.2067759372 \quad 0.285235387$

$7.0705484107-0.914935542$

$5.8074823678-1.4767105725$

$3.2740899526 \quad 1.9259961569$

$2.8142261546-2.1720195724$

$6.1824676333 \quad 1.8919375137$

$8.1977906419 \quad 0.6989726224$

$7.9573649331-1.4166518872$

$5.6938429587-2.4091435041$

\section{8 (syn, syn)}

$E=-1033.7516723600$ Hartree

$\begin{array}{lrrr}\text { C } & 0.2674765327 & 1.2870342992 & -0.2586107117\end{array}$

$\begin{array}{llll}\mathrm{N} & -0.9032872174 & 0.7235630934 & 0.1215311684\end{array}$

$\mathrm{N} \quad 1.2891179403 \quad 0.556271616-0.7175186249$

C $\quad 0.3879011242 \quad 2.6969878172 \quad-0.197603353$

$\mathrm{H}-1.6254861472 \quad 1.41299817130 .3421756162$

$\mathrm{H} \quad 2.1068176475 \quad 1.1213330857-0.953462475$

C $\quad 1.2094854825-0.7554149636-1.3596184745$

C $\quad 1.952473384-0.7182589835-2.6937775637$

C $\quad 1.4345633232 \quad 0.35334731-3.6572658117$

C $-0.0619288595 \quad 0.2421480523-3.9396385468$

$\mathrm{H} \quad 0.1574972707 \quad-1.0039420976-1.5167225897$

$\mathrm{H} \quad 1.6350868772-1.5238248973-0.7050637093$

$\mathrm{H} \quad 1.8540511309-1.7105079355-3.1501509801$

$\mathrm{H} \quad 3.0212686555 \quad-0.5593141434-2.5031237049$

$\mathrm{H} \quad 1.9924696919 \quad 0.2700567344 \quad-4.596763424$

$\mathrm{H} \quad 1.659364895 \quad 1.3483876395 \quad-3.2524921318$

H $-0.3213172712 \quad-0.7631672539-4.2918859499$

$\mathrm{H}-0.3694444431 \quad 0.9578071677-4.7082728863$

H $-0.6597834975 \quad 0.4455044845 \quad-3.0437234178$

$\begin{array}{llll}\text { C }-1.1228265632 & -0.5798014234 & 0.6389660317\end{array}$

$\begin{array}{llll}\text { C }-0.6233720394 & 3.6011584652 & 0.2990758602\end{array}$

C $-0.0660921229 \quad 4.9904070862 \quad 0.1412090399$

C $\quad 1.2025381534 \quad 4.901833026 \quad-0.4342245994$

C $\quad 1.5243821239 \quad 3.4509599262 \quad-0.67222326$

$\begin{array}{llll}\text { C }-0.6263016893 & 6.2124022333 & 0.4664972072\end{array}$

$\begin{array}{llll}\text { C } & 0.1259940897 & 7.3639840627 & 0.2003966821\end{array}$

$\begin{array}{llll}\text { C } & 1.3947960974 & 7.2755286087 & -0.3745697512\end{array}$

C $\quad 1.952657026 \quad 6.0326980646 \quad-0.7018176605$

$\begin{array}{lllll}0 & -1.7426876865 & 3.3405425037 & 0.7426547037\end{array}$

o $2.5742743059 \quad 3.0365573843-1.1679477406$

$\mathrm{H}-1.6146368909 \quad 6.2750879489 \quad 0.9124858287$

H $-0.2820174937 \quad 8.3402398947 \quad 0.4440067646$

$\mathrm{H} \quad 1.955524906 \quad 8.1843064551 \quad-0.5709867634$

$\mathrm{H} \quad 2.9388706957 \quad 5.9580125404-1.150600795$

C $-0.1401114865-1.2699359861 \quad 1.3537709923$

C $-0.4276620031-2.5288615381 \quad 1.8745255805$

$\begin{array}{llll}\text { C }-1.6902782946 & -3.0949579963 & 1.7075844163\end{array}$

C $-2.6723185058-2.3917528208 \quad 1.011376909$ 


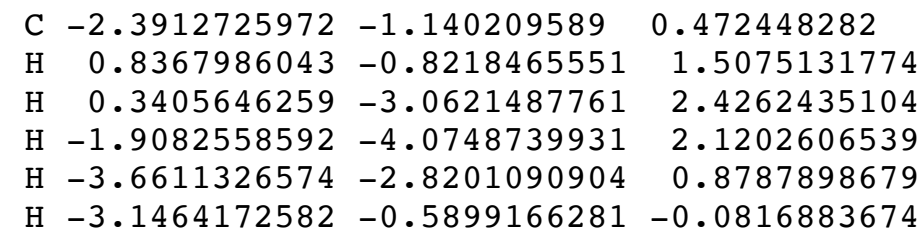

8 (anti,anti)

$E=-1033.7381634900$ Hartree

C $0.2292164408 \quad 1.420141348 \quad-0.3387429818$

$\mathrm{N}-0.9254650207 \quad 0.7853433506 \quad-0.0049738108$

$\mathrm{N} \quad 1.21893737 \quad 0.6181899774 \quad-0.750202554$

C $0.3864304341 \quad 2.8201106249 \quad-0.2322832663$

H $-0.8394780895-0.2020906726 \quad 0.2211141659$

H $\quad 0.9633120419-0.3431306348 \quad-0.9624575984$

C $2.6500630918 \quad 0.8869732863 \quad-0.6571024444$

C $3.3602869038-0.2744457867 \quad 0.0352440753$

C $2.8256026154-0.5801095188 \quad 1.4368819248$

$\begin{array}{llll}\text { C } & 2.8525964702 & 0.62609695 & 2.3729868464\end{array}$

$\mathrm{H} \quad 2.7763963897 \quad 1.8125831982 \quad-0.0925735622$

H $\quad 3.0581951802 \quad 1.0583130182-1.6564387665$

H $\quad 4.4254242443-0.01862068320 .0914862316$

$\mathrm{H} \quad 3.2825437652-1.1702612991-0.5939656469$

$\mathrm{H} \quad 3.4242683779-1.3914502805 \quad 1.8661820003$

$\mathrm{H} \quad 1.7989697347-0.9612039094 \quad 1.3633906618$

$\mathrm{H} \quad 3.8549817267 \quad 1.0682157342 \quad 2.4147495596$

$\mathrm{H} \quad 2.5687664486 \quad 0.3389412596 \quad 3.3901572506$

H $2.157406786 \quad 1.4078860019 \quad 2.0458128441$

C $-2.2501763116 \quad 1.2740831731 \quad-0.0406071197$

$\begin{array}{llll}\text { C }-0.2963595614 & 3.6736373588 & 0.730116616\end{array}$

C $\quad 0.1457458727 \quad 5.0825363665 \quad 0.4261894116$

C $1.0201300457 \quad 5.0613965472 \quad-0.657587239$

C $1.2040720068 \quad 3.6363026428-1.1144922713$

$\begin{array}{llll}\text { C }-0.199485889 & 6.2690064916 & 1.0510808877\end{array}$

$\begin{array}{llll}\text { C } & 0.3576759241 & 7.4528556937 & 0.553682523\end{array}$

C $1.2329815503 \quad 7.4317710816-0.5351476186$

C $1.57641509516 .2263646765-1.158329094$

$\begin{array}{llll}0 & -1.0555625929 & 3.3751604291 & 1.6424521097\end{array}$

$\begin{array}{llll}0 & 1.8904136529 & 3.3075363745 & -2.0756307578\end{array}$

$\mathrm{H}-0.8823216283 \quad 6.2777026074 \quad 1.8958763669$

$\mathrm{H} \quad 0.1068820561 \quad 8.4021188641 \quad 1.0175941108$

$\mathrm{H} \quad 1.6514689082 \quad 8.3649118388-0.9004296843$

$\mathrm{H} \quad 2.2571572024 \quad 6.2023401707-2.0045492841$

C $-2.655307183 \quad 2.2956438123 \quad-0.9038280213$

C $-3.9877350926 \quad 2.6962576566 \quad-0.914660504$

C $-4.9275246529 \quad 2.0775623171-0.0910421593$

C $-4.5210014025 \quad 1.0447197301 \quad 0.7511574911$

$\begin{array}{llll}\text { C }-3.1884808052 & 0.6451083477 & 0.7807793569\end{array}$

$\mathrm{H}-1.9361571847 \quad 2.7710684346-1.5619173876$

$\mathrm{H}-4.2937439605 \quad 3.4935366736-1.5855355824$

$\mathrm{H}-5.9656397379 \quad 2.3938168236-0.1103486561$

$\mathrm{H}-5.240438061 \quad 0.5478077296 \quad 1.3950092848$

$\mathrm{H}-2.8656421618-0.1538378056 \quad 1.4430132918$

\section{9 (syn, syn)}

$E=-1107.5384217300$ Hartree

C $\quad 0.4072765448 \quad 1.3719734655 \quad 0.2293293691$

$\begin{array}{llll}\mathrm{N} & -0.7379328624 & 0.9075551542 & 0.7699935097\end{array}$

$\mathrm{N} \quad 1.4221523985 \quad 0.5658717674 \quad-0.1444833495$

C $0.550275006 \quad 2.7653537262 \quad 0.0464475963$

$\mathrm{H}-1.4472698148 \quad 1.6341051724 \quad 0.8945265506$

H $2.2588170729 \quad 1.0797362023 \quad-0.431673747$

C $-0.9763844792 \quad-0.3263514393 \quad 1.427281459$ 


$\begin{array}{lccc}\mathrm{C} & -0.4355853561 & 3.7586617085 & 0.4096099763 \\ \mathrm{C} & 0.1469255097 & 5.0931455484 & 0.0328561037 \\ \mathrm{C} & 1.410658869 & 4.8902975907 & -0.5243762412 \\ \mathrm{C} & 1.7047886544 & 3.4153295838 & -0.5324221869 \\ \mathrm{C} & -0.3863615914 & 6.3624463948 & 0.1667404646 \\ \mathrm{C} & 0.3878958547 & 7.4426540035 & -0.2759003634 \\ \mathrm{C} & 1.6520257419 & 7.2398693252 & -0.8323380338 \\ \mathrm{C} & 2.1830385045 & 5.9503139898 & -0.9639661984 \\ \mathrm{O} & -1.5500524825 & 3.5911610765 & 0.9060468999 \\ \mathrm{O} & 2.7499177798 & 2.9074712476 & -0.9402540171 \\ \mathrm{H} & -1.3704243028 & 6.5140743415 & 0.6003647234 \\ \mathrm{H} & 0.0009384518 & 8.453065956 & -0.1856542237 \\ \mathrm{H} & 2.2305079433 & 8.0953559829 & -1.1674702371 \\ \mathrm{H} & 3.1658434191 & 5.7864435621 & -1.3959862643 \\ \mathrm{C} & 0.0066699136 & -0.9513887575 & 2.1978056057 \\ \mathrm{C} & -0.2766110714 & -2.1666785921 & 2.8133601831 \\ \mathrm{C} & -1.5390225965 & -2.7461107978 & 2.6902283437 \\ \mathrm{C} & -2.5250993258 & -2.0996831609 & 1.9470714233 \\ \mathrm{C} & -2.2457005068 & -0.8941259966 & 1.309941453 \\ \mathrm{H} & 0.984874326 & -0.4932893844 & 2.3070958595 \\ \mathrm{H} & 0.4934877768 & -2.6567121915 & 3.4012804982 \\ \mathrm{H} & -1.753762098 & -3.6929978375 & 3.1754624264 \\ \mathrm{H} & -3.5135550237 & -2.5383400401 & 1.8510251187 \\ \mathrm{H} & -2.999560264 & -0.3925583332 & 0.7095757709 \\ \mathrm{C} & 1.3922000565 & -0.816893443 & -0.4583040792 \\ \mathrm{C} & 2.5251531625 & -1.5774498047 & -0.1662910655 \\ \mathrm{C} & 0.2844953262 & -1.4087165348 & -1.069466416 \\ \mathrm{C} & 0.3032981228 & -2.7721520549 & -1.3465491582 \\ \mathrm{C} & 1.4272998639 & -3.5408810497 & -1.0461157817 \\ \mathrm{C} & 2.5415364275 & -2.9364152141 & -0.4668105281 \\ \mathrm{H} & 3.3793044883 & -1.1001314471 & 0.3058381112 \\ \mathrm{H} & -0.5849866489 & -0.8079747889 & -1.3180395619 \\ \mathrm{H} & -0.5641213792 & -3.2321339104 & -1.8100531379 \\ \mathrm{H} & 1.4359495166 & -4.6034391136 & -1.2676014452 \\ \mathrm{H} & 3.4246180723 & -3.5241909075 & -0.2357784103\end{array}$

9 (anti, anti)

$\mathbf{E}=-1107.5228130600$ Hartree

$\begin{array}{llll}\text { C } & 0.4102682769 & 1.3998932332 & 0.2256996173\end{array}$

$\begin{array}{llll}\mathrm{N}-0.7648962138 & 0.8112982677 & 0.5617803097\end{array}$

$\begin{array}{llll}\mathrm{N} & 1.4350183522 & 0.522602385 & 0.0819893774\end{array}$

C $\quad 0.5521558489 \quad 2.7825929733 \quad 0.0442356502$

$\mathrm{H}-0.691963143-0.10441310160 .9969260685$

H $\quad 1.1693285619-0.4402300501-0.1065899436$

$\begin{array}{llll}\text { C }-2.0864517483 & 1.2312031895 & 0.2869515962\end{array}$

$\begin{array}{llll}\text { C }-0.2472908213 & 3.8076120597 & 0.7086192582\end{array}$

$\begin{array}{llll}\text { C } & 0.2654465928 & 5.1303489781 & 0.2045041859\end{array}$

C $1.2975723762 \quad 4.9053539186-0.7028144296$

C $\quad 1.5225237281 \quad 3.4229227899 \quad-0.8386824887$

$\begin{array}{llll}\text { C }-0.1476326679 & 6.4134901373 & 0.5239335966\end{array}$

C $0.505453799 \quad 7.4810375272-0.1012906185$

C $1.5398005417 \quad 7.2550999338-1.0140537333$

C $1.9499928297 \quad 5.9551161621-1.3284881732$

$\begin{array}{llll}0 & -1.134996846 & 3.6862660729 & 1.5406585454\end{array}$

$\begin{array}{lllll} & 2.3459916131 & 2.918355618 & -1.5885816218\end{array}$

$\mathrm{H}-0.9533205097 \quad 6.5812346297 \quad 1.2328190567$

H $\quad 0.2067169579 \quad 8.5005521503 \quad 0.1232761735$

H $2.0300753762 \quad 8.102536515-1.4836981405$

$\mathrm{H} \quad 2.7540901044 \quad 5.7718612649 \quad-2.0353366847$

C $-2.4080872796 \quad 2.0482172308 \quad-0.7997996129$

C $-3.7382109523 \quad 2.3783046776-1.0381462315$

C $-4.7543487049 \quad 1.8908718516-0.2167280137$

C $-4.427752954 \quad 1.0611894134 \quad 0.8537626105$ 

C -3.0998681173
$\mathrm{H}-1.6270385063$
$0.7326035124 \quad 1.1084643008$
$\mathrm{H}-3.9806755136$
$2.4206807481-1.4536334081$
$\mathrm{H}-5.7895199851$
$3.0157044548-1.8832045834$
$\mathrm{H}-5.2071409276$
$2.1516412901-0.4128475139$
$\mathrm{H}-2.8396284143$
$0.6680029881 \quad 1.4995138145$
C 2.8205503641
$0.090739418 \quad 1.9460495898$
C 3.6920938609
$0.7261568008 \quad 0.2749223785$
$\begin{array}{llll}\text { C } & 3.3272455377 & 1.7124395241 & 1.1249198144\end{array}$
$\begin{array}{llll}\text { C } & 4.7022815349 & 1.8200025271 & 1.3057577596\end{array}$
$\begin{array}{llll}\text { C } & 5.5787222556 & 0.9461733209 & 0.6630066905\end{array}$
C $5.0650660457-0.0469451452-0.1681743028$
$\mathrm{H} \quad 3.2870464318 \quad-0.9269088144 \quad-1.0174782318$
H $\quad 2.6537247043 \quad 2.3877606993 \quad 1.6412297458$

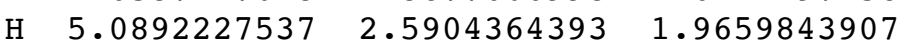
H $\quad 6.6498996333 \quad 1.0362685905 \quad 0.8130229966$
H $\quad 5.733035224 \quad-0.7394710593 \quad-0.6714249131$ 


\section{X-ray crystallography}

\section{MN1}

MN1 crystals were prepared from slow evaporation at room temperature from MeCN. Thermal ellipsoids are shown at the $50 \%$ probability level.
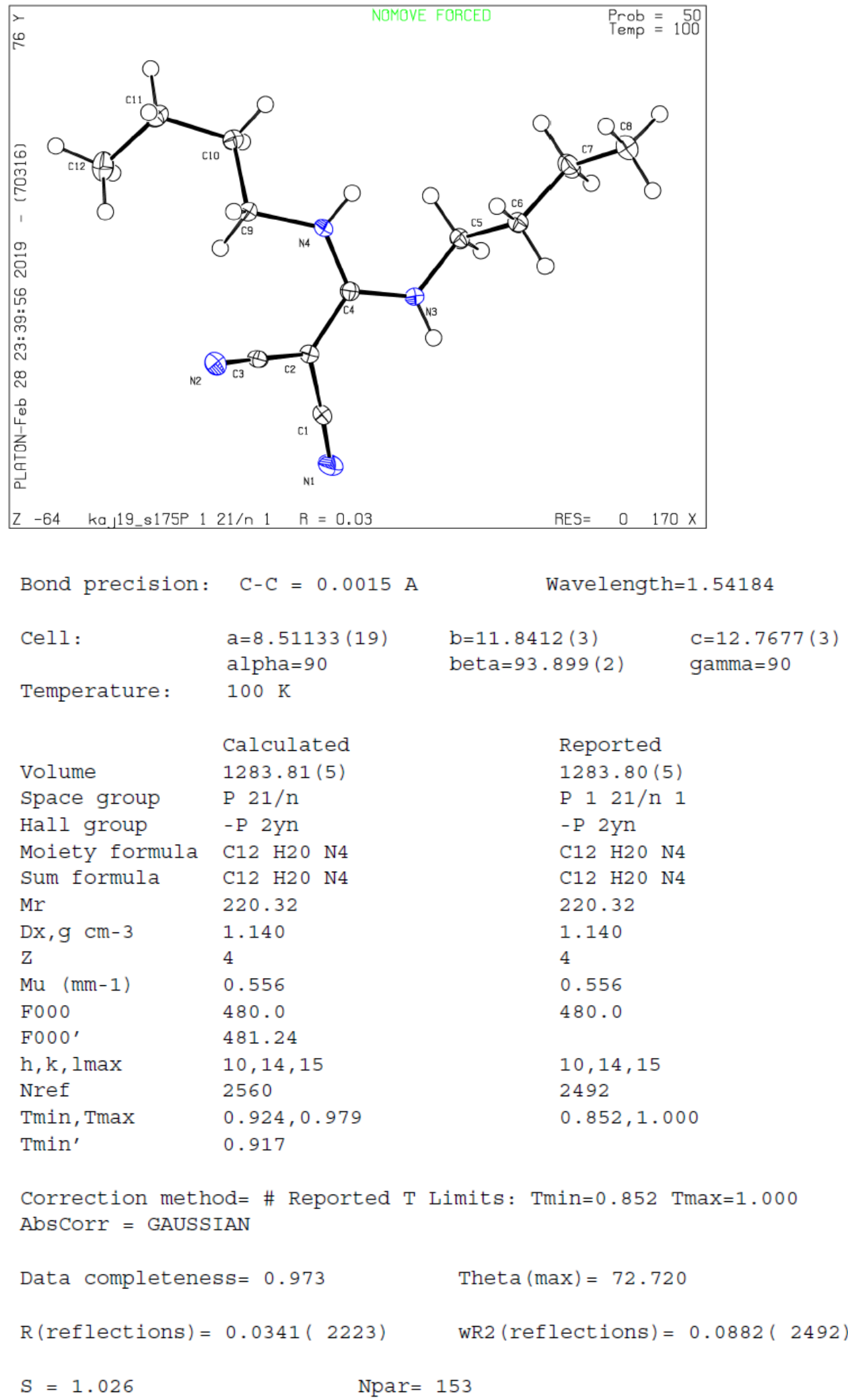

Tmin'

Correction method= \# Reported T Limits: Tmin $=0.852$ Tmax $=1.000$ AbsCorr = GAUSSIAN

Data completeness $=0.973$

$\mathrm{R}($ reflections $)=0.0341(2223)$

$S=1.026$

Theta $(\max )=72.720$

WR2 $($ reflections $)=0.0882(2492)$ 
MN2

MN2 crystals were prepared from slow evaporation at room temperature from MeCN. Thermal ellipsoids are shown at the $50 \%$ probability level.

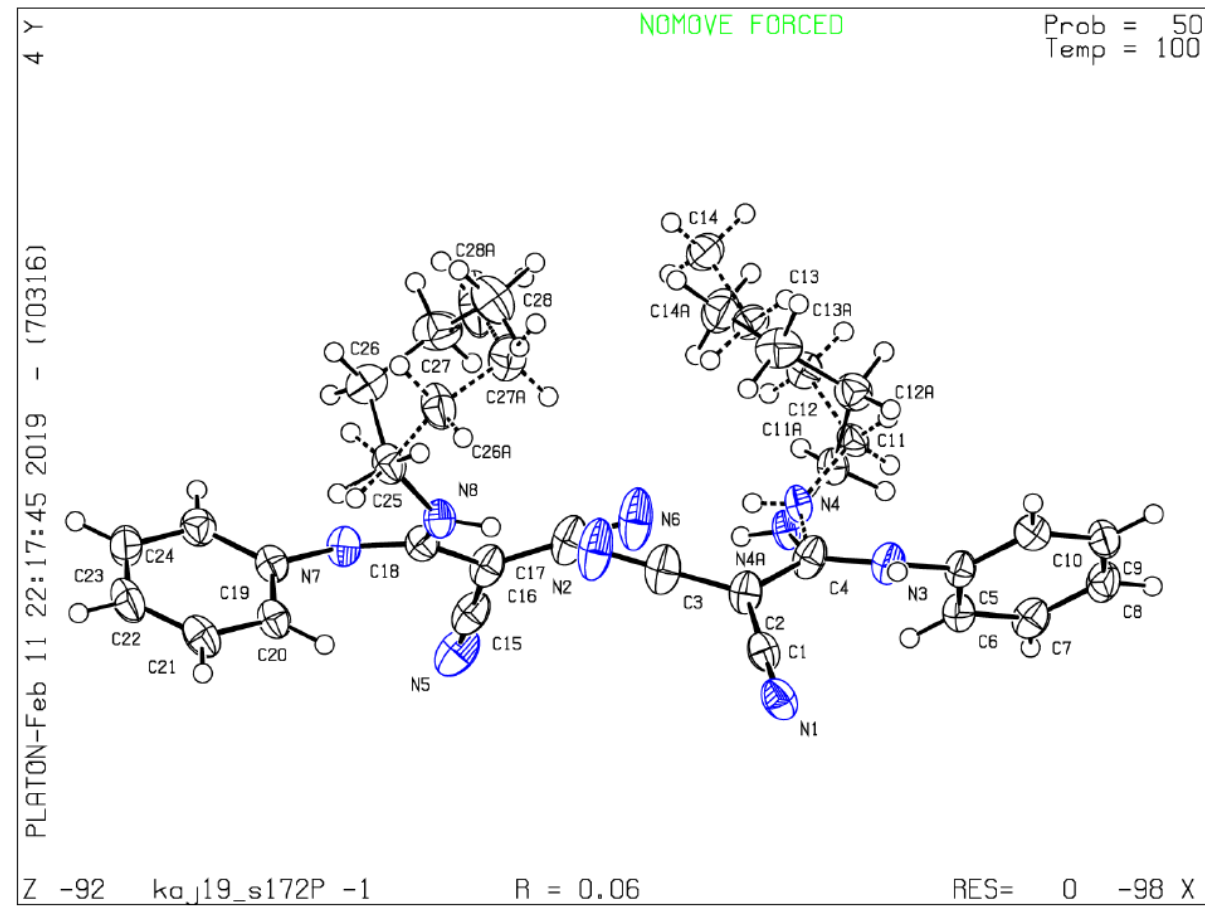

Bond precision: $\quad \mathrm{C}-\mathrm{C}=0.0030 \mathrm{~A} \quad$ Wavelength $=1.54184$

$\begin{array}{llll}\text { Cell: } & \begin{array}{l}\mathrm{a}=7.3876(4) \\ \mathrm{alpha}=66.771(7)\end{array} & \mathrm{b}=13.9227(9) & \mathrm{c}=14.5943(12) \\ \text { Temperature: } & 100 \mathrm{~K} & & \end{array}$

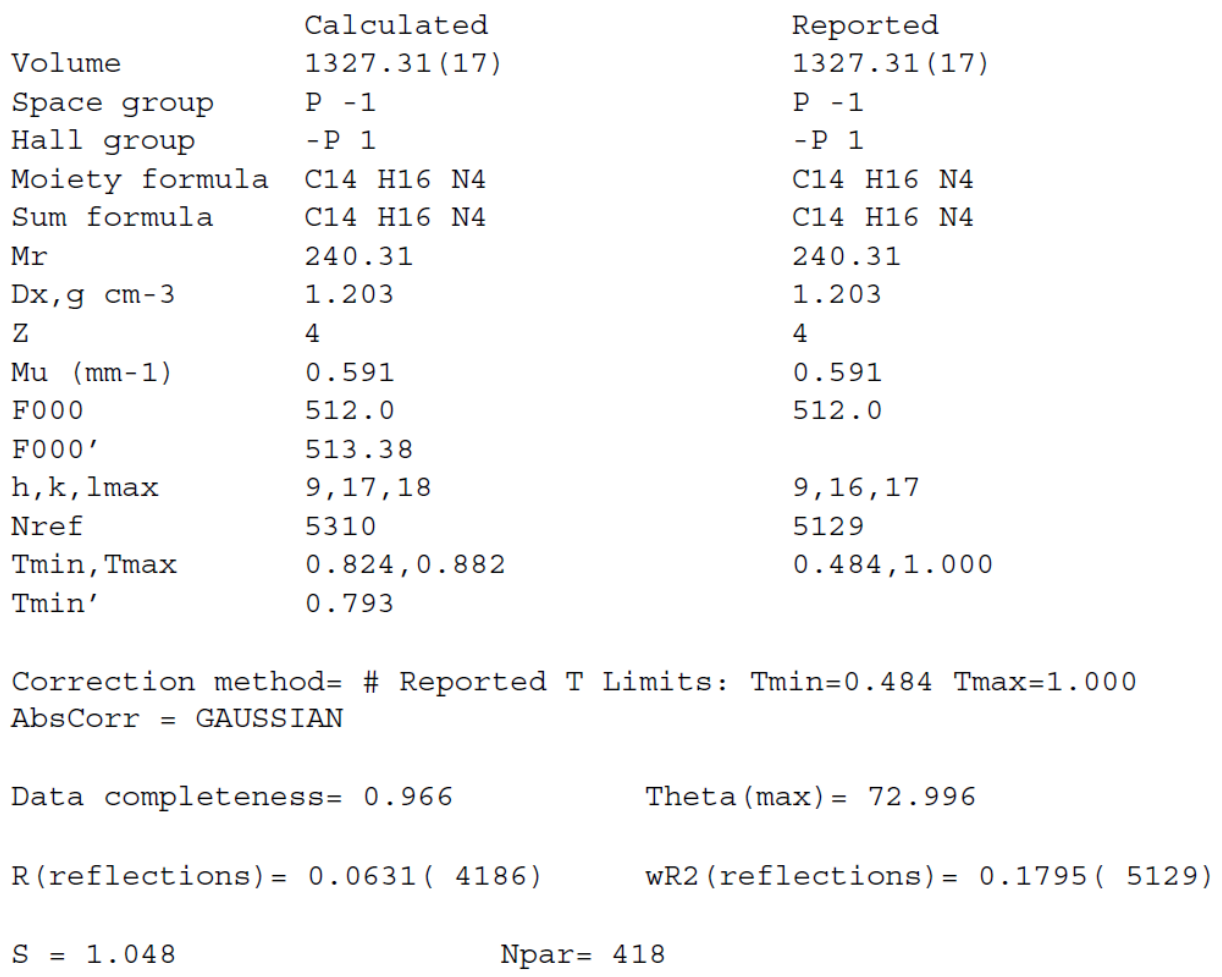


MN3

MN3 crystals were prepared from slow evaporation at room temperature from MeCN. Thermal ellipsoids are shown at the $50 \%$ probability level.

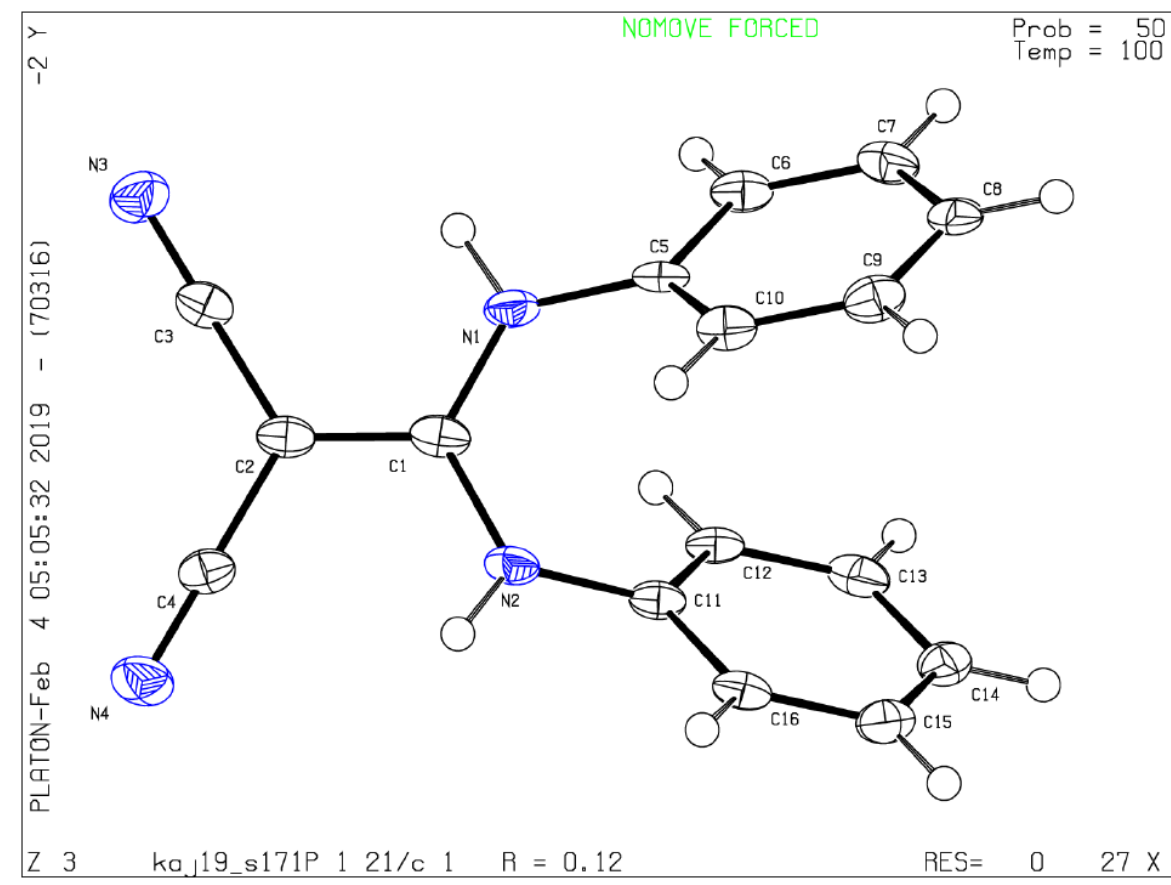

\begin{tabular}{|c|c|c|c|}
\hline Bond precision: & $C-C=0.0103 \mathrm{~A}$ & Wavelengt $\mathrm{h}=1$ & .54184 \\
\hline Cell: & $\begin{array}{l}a=14.7711(9) \\
a l p h a=90\end{array}$ & $\begin{array}{l}\mathrm{b}=11.8386(7) \\
\text { bet } a=95.352(5)\end{array}$ & $\begin{array}{l}\mathrm{C}=7.4894(3) \\
\text { gamma }=90\end{array}$ \\
\hline \multirow[t]{2}{*}{ Temperature: } & $100 \mathrm{~K}$ & & \\
\hline & Calculated & Reported & \\
\hline Volume & $1303.96(12)$ & $1303.95(13)$ & \\
\hline Space group & P $21 / C$ & P $121 / C 1$ & \\
\hline Hall group & $-\mathrm{P} 2 \mathrm{ybc}$ & $-\mathrm{P} 2 \mathrm{ybc}$ & \\
\hline Moiety formula & C16 H12 N4 & $\mathrm{C} 16 \mathrm{H} 12 \mathrm{~N} 4$ & \\
\hline Sum formula & C16 H12 N4 & $\mathrm{C} 16 \mathrm{H} 12 \mathrm{~N} 4$ & \\
\hline $\mathrm{Mr}$ & 260.30 & 260.30 & \\
\hline$D x, g c m-3$ & 1.326 & 1.326 & \\
\hline $\mathrm{Z}$ & 4 & 4 & \\
\hline $\mathrm{Mu} \quad(\mathrm{mm}-1)$ & 0.656 & 0.656 & \\
\hline F000 & 544.0 & 544.0 & \\
\hline $\mathrm{F} 000^{\prime}$ & 545.52 & & \\
\hline$h, k, l \max$ & $17,14,8$ & $17,14,8$ & \\
\hline Nref & 2305 & 2302 & \\
\hline Tmin, Tmax & $0.878,0.986$ & $0.730,1.000$ & \\
\hline Tmin' & 0.820 & & \\
\hline
\end{tabular}

Correction method $=\#$ Reported T Limits: Tmin=0.730 Tmax $=1.000$ AbsCorr = GAUSSIAN

Data completeness $=0.999$

Theta $(\max )=66.589$

$R($ reflections $)=0.1223(1960)$

wR2 (reflections $)=0.3751(2302)$

$S=1.171$

Npar $=188$ 
ID1

ID1 crystals were prepared from slow evaporation at room temperature from MeCN. Thermal ellipsoids are shown at the $50 \%$ probability level.

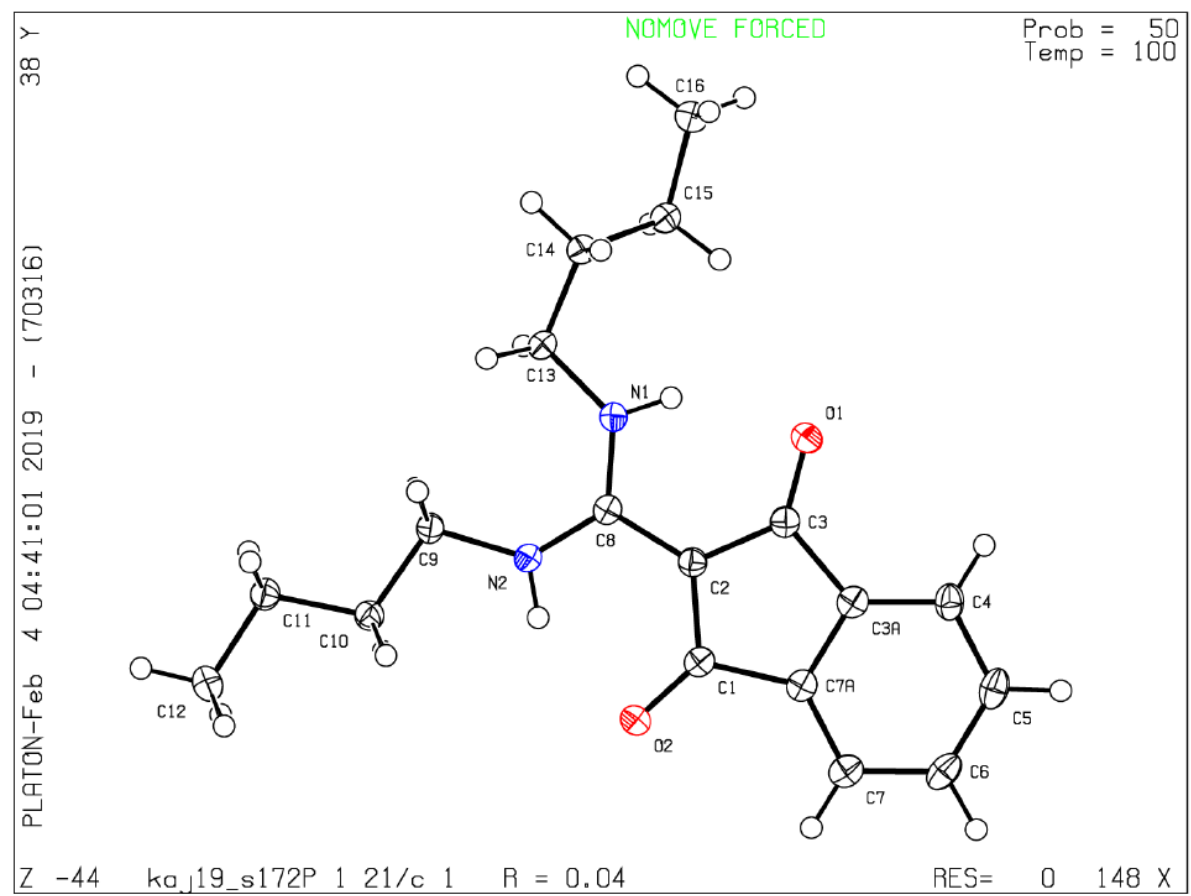

\begin{tabular}{|c|c|c|c|}
\hline Bond precision: & $C-C=0.0019 \mathrm{~A}$ & Wavelength $=1$ & $=1.54184$ \\
\hline Cell: & $\begin{array}{l}a=12.1477(6) \\
\text { alpha }=90\end{array}$ & $\begin{array}{l}\mathrm{b}=8.2277(4) \\
\mathrm{bet} a=96.783(5)\end{array}$ & $\begin{array}{l}\mathrm{C}=16.3837(8) \\
\text { gamma }=90\end{array}$ \\
\hline \multirow[t]{2}{*}{ Temperature: } & $100 \mathrm{~K}$ & & \\
\hline & Calculated & Reported & \\
\hline Volume & $1626.05(14)$ & $1626.06(13)$ & \\
\hline Space group & P $21 / \mathrm{C}$ & $\mathrm{P} 121 / \mathrm{C} 1$ & \\
\hline Hall group & $-\mathrm{P} 2 \mathrm{ybc}$ & $-\mathrm{P} 2 \mathrm{ybc}$ & \\
\hline Moiety formula & $\mathrm{C} 18 \mathrm{H} 24 \quad \mathrm{~N} 2 \mathrm{O} 2$ & C18 H24 N2 & $\mathrm{O} 2$ \\
\hline Sum formula & $\mathrm{C} 18$ H24 N2 O2 & C18 H24 N2 & $\mathrm{O} 2$ \\
\hline $\mathrm{Mr}$ & 300.39 & 300.39 & \\
\hline $\mathrm{Dx}, \mathrm{gcm}-3$ & 1.227 & 1.227 & \\
\hline $\mathrm{z}$ & 4 & 4 & \\
\hline $\mathrm{Mu} \quad(m m-1)$ & 0.637 & 0.637 & \\
\hline F000 & 648.0 & 648.0 & \\
\hline F000' & 649.84 & & \\
\hline$h, k, I \max$ & $15,10,20$ & $15,10,20$ & \\
\hline Nref & 3241 & 3148 & \\
\hline Tmin, Tmax & $0.954,0.969$ & $0.514,1.000$ & \\
\hline Tmin' & 0.713 & & \\
\hline
\end{tabular}

Correction method= \# Reported T Limits: Tmin=0.514 Tmax=1.000 AbsCorr = GAUSSIAN

Data completeness $=0.971$

Theta $(\max )=72.765$

$R($ reflections $)=0.0388(2708)$

$\mathrm{WR} 2($ reflections $)=0.1040(3148)$

$S=1.044$

Npar $=207$ 
ID3

ID3 crystals were prepared from slow evaporation at room temperature from MeCN. Thermal ellipsoids are shown at the $50 \%$ probability level.
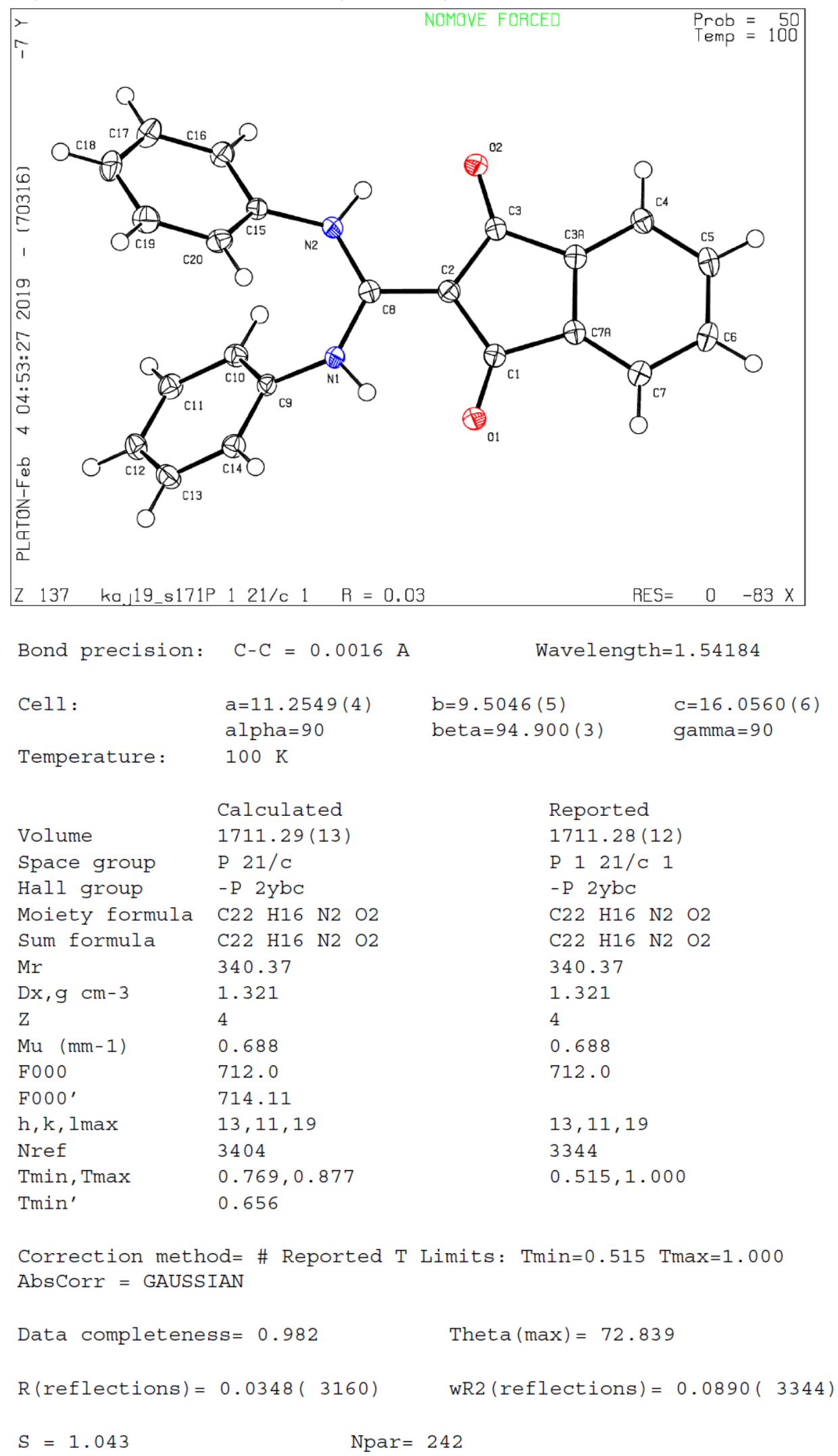

Correction method= \# Reported T Limits: Tmin=0.515 Tmax $=1.000$ AbsCorr = GAUSSIAN

Data completeness $=0.982$ Theta $(\max )=72.839$

$\mathrm{R}($ reflections $)=0.0348(3160$ 


\section{References}

1. P. Thordarson, Determining association constants from titration experiments in supramolecular chemistry, Chem. Soc. Rev., 2011, 40 , 1305-1323

2. Gaussian 16, Revision B.01, Frisch, M. J.; Trucks, G. W.; Schlegel, H. B.; Scuseria, G. E.; Robb, M. A.; Cheeseman, J. R.; Scalmani, G.; Barone, V.; Petersson, G. A.; Nakatsuji, H.; Li, X.; Caricato, M.; Marenich, A. V.; Bloino, J.; Janesko, B. G.; Gomperts, R.; Mennucci, B.; Hratchian, H. P.; Ortiz, J. V.; Izmaylov, A. F.; Sonnenberg, J. L.; Williams-Young, D.; Ding, F.; Lipparini, F.; Egidi, F.; Goings, J.; Peng, B.; Petrone, A.; Henderson, T.; Ranasinghe, D.; Zakrzewski, V. G.; Gao, J.; Rega, N.; Zheng, G.; Liang, W.; Hada, M.; Ehara, M.; Toyota, K.; Fukuda, R.; Hasegawa, J.; Ishida, M.; Nakajima, T.; Honda, Y.; Kitao, O.; Nakai, H.; Vreven, T.; Throssell, K.; Montgomery, J. A., Jr.; Peralta, J. E.; Ogliaro, F.; Bearpark, M. J.; Heyd, J. J.; Brothers, E. N.; Kudin, K. N.; Staroverov, V. N.; Keith, T. A.; Kobayashi, R.; Normand, J.; Raghavachari, K.; Rendell, A. P.; Burant, J. C.; Iyengar, S. S.; Tomasi, J.; Cossi, M.; Millam, J. M.; Klene, M.; Adamo, C.; Cammi, R.; Ochterski, J. W.; Martin, R. L.; Morokuma, K.; Farkas, O.; Foresman, J. B.; Fox, D. J. Gaussian, Inc., Wallingford CT, 2016.

3. Zhao, Y.; Truhlar, D. G. Theor., The M06 suite of density functionals for main group thermochemistry, thermochemical kinetics, noncovalent interactions, excited states, and transition elements: two new functionals and systematic testing of four M06-class functionals and 12 other functionals, Theor. Chem. Acc. 2008, 120, 215-241.

4. Marenich, A. V.; Cramer, C. J.; Truhlar, D. G., Universal Solvation Model Based on Solute Electron Density and on a Continuum Model of the Solvent Defined by the Bulk Dielectric Constant and Atomic Surface Tensions, J. Phys. Chem. B 2009, 113, 6378-6396. 